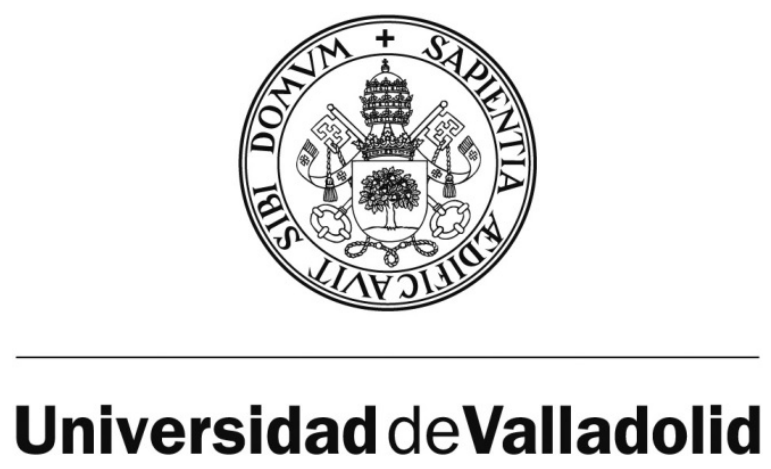

ESCUELA TÉCNICA SUPERIOR DE INGENIEROS DE TELECOMUNICACIÓN DEPARTAMENTO DE TEORÍA DE LA SEÑAL Y COMUNICACIONES E INGENIERÍA TELEMÁTICA

TESIS DOCTORAL:

\title{
DECODING P300 EVOKED POTENTIALS FOR BRAIN COMPUTER INTERFACES (BCI) AIMED AT ASSISTING POTENTIAL END-USERS AT HOME
}

Presentada por Rebeca Corralejo Palacios para optar al grado de doctora por la Universidad de Valladolid

Dirigida por:

Roberto Hornero Sánchez 



\section{Acknowledgements}

First of all, I would like to express my sincere gratitude to my supervisor, Dr. Hornero, for his helpful advices, suggestions and reinforcement during the course of this work.

I would also like to thank all the members of the Grupo de Ingeniería Biomédica for their continued support over these years. Especially, to María and Javier, who shared a lot of cups of coffee with me in the early morning.

Furthermore, I am really grateful to the staff of the Centro de Referencia Estatal para la Atención a Personas con Grave Discapacidad y para la Promoción de la Autonomía Personal y Atención a la Dependencia, especially to its former and actual managers, Nuria Mendoza and María Teresa Gutiérrez, respectively. Of course, I would also like to thank the users of this centre who kindly took part in our study. Without their collaboration, this Doctoral Thesis could not have been carried out.

Finally, I would like to express my gratitude to my parents, Isaac and María Jesús; my sisters, Ana María e Isabel; and my partner, Fernando; who have continuously supported and encouraged me, even from the distance.

This research was partly supported by the Consejería de Educación (Junta de Castilla y León) and the European Social Fund within the grant program Personal Investigador de Reciente Titulación Universitaria (PIRTU); and by the Project Cero 2011 on Ageing funded by Fundación General CSIC, Obra Social La Caixa and CSIC. 

A Brain Computer Interface (BCI) is a communication system that provides an alternative channel to natural communication and control processes since it bypasses the body's normal efferent pathways, which are the neuromuscular output channels. Hence, a BCI system monitors brain activity and translates specific signal features, which reflect the user's intent, into commands that operate a device. BCIs are especially interesting for severely impaired individuals that cannot perform physical movements. Conventional augmentative and alternative communication technologies require some form of muscle control and thus may not be useful for those with the most severe motor disabilities, such as late-stage amyotrophic lateral sclerosis (ALS), brainstem stroke or severe cerebral palsy.

This Thesis proposes the use of P300 evoked potentials as signal control in BCI systems for end-users, i.e. severely impaired people. Selective attention to a specific and infrequent flashing symbol evokes a brain pattern called P300, which appears around the central and parietal brain cortex, about $300 \mathrm{~ms}$ after the stimulus presentation. A novel assistive BCI tool for environment control at home is researched. The proposed application is based on P300 evoked responses to infrequent stimuli, known as the 'oddball' paradigm. P300-based BCIs could be the most proper type of BCIs for severely disabled populations since it does not require training period. Furthermore, its typical paradigm allows to select in a fast manner the desired symbol among a large number of choices only by focusing on it. The methodology proposed in this work is focused on the real end-users. Thus, the design, the experiments and the assessment are centred on the potential end-users' needs.

A population of 30 potential BCI end-users, people with severe impairments due to different pathologies and degree of both motor and cognitive disabilities, took part in the study. Participants were recruited through the Spanish National Reference Centre on Disability and Dependence (León, Spain). The main needs identified for the population under study were related to three main categories: comfort, communication and security. Hence, the proposed assistive BCI tool was designed to cover them. Specifically, it enables to manage 8 devices, usually present at home, by means of 115 
infrared control commands and 22 navigation commands. In order to simplify the interface, these commands were distributed among ten matrices: a main menu and nine sub-menus. In the experimental phase, the individuals interacted with the assistive tool during three sessions managing real electronic devices. Twenty three out of the thirty subjects were able to suitably manage the proposed BCI tool with accuracy higher than 80\%. Nineteen out of them even achieved accuracy above 95\%. Moreover, maximum rates up to $6.8 \mathrm{item} / \mathrm{min}$ and $25.9 \mathrm{bit} / \mathrm{min}$ were reached. These results show that most of potential end-users would be able to manage typical P300-based BCIs without special considerations. Furthermore, the analysis of the results suggested that the degree of impairment would not be a relevant issue in order to properly manage a P300-based BCI tool, since it seemed not to have an influence on the performance of individuals managing the proposed assistive tool. Statistical significance was not reached $(p>0.05)$ between the online BCI performance and the degree of motor impairment, cognitive impairment and sustained attention ability of the thirty participants. Therefore, these applications could be appropriate for assisting and increasing the personal autonomy of severely impaired subjects at home. Furthermore, the proposed tool could easily increase and adapt the range of needs that can be satisfied for each specific patient. Thus, the dependence from caregivers, relatives and nurses could be reduced for disabled populations.

The end-users' assessment was carried out by means of a questionnaire. It showed that BCI potential users are willing to take part in this kind of studies, providing their requirements and their point of view. Hence, future works should focus on endusers for designing, developing and assessing assistive BCI applications. The population under study showed its satisfaction with the proposed assistive BCI tool through the assessment questionnaires. Most of the participants rated favourably the design and usefulness of the assistive tool for daily use at home. More specifically, users stated that the proposed tool was easy to manage and understand. Furthermore, participants considered that the proposed tool was really useful to increase their autonomy at home. They were even interested in including more home devices such as blinds, doors or bells; whereas they were not especially interested in BCIs for mobility applications. Nevertheless, individuals stated that electroencephalogram (EEG)-based BCIs are not practical on a daily basis. Participants suggested the use of wireless caps and dry electrodes in order to make EEG recordings more comfortable and practical for 
its regular use at home. Hence, future BCI developments for disabled populations should take these suggestions from real BCI end-users into consideration.

In this Thesis, a novel P300-based assistive BCI tool for severely impaired people was investigated. Not only the design but also the experimental procedure and the evaluation were carried out through real end-users. Moreover, the experimental stage was performed with real devices and in the patients' usual environment. Therefore, this study takes a noteworthy step considering the opinion and feelings of end-users as well as their degree of satisfaction. 

Una interfaz cerebro-ordenador o Brain Computer Interface (BCI) es un sistema de comunicación que proporciona un canal alternativo a los procesos de comunicación y control naturales, ya que no emplea las conexiones neuromusculares, que son los canales de salida normales del cuerpo humano. Por tanto, lo que hace un sistema BCI es monitorizar la actividad cerebral y traducir características específicas de la misma, relacionadas con la intención del usuario, en comandos que permiten controlar un dispositivo. Los sistemas BCI son especialmente interesantes en el caso de personas con grave discapacidad que les impide la realización de movimientos. Las tecnologías de comunicación aumentativas y alternativas convencionales necesitan de algún tipo de control muscular y precisamente por ello pueden no resultar útiles para aquellas personas con discapacidad motora severa, como los afectados por esclerosis lateral amiotrófica (ELA) en estadio avanzado, por apoplejía o por parálisis cerebral grave.

La presente Tesis Doctoral propone el empleo de potenciales evocados P300 como señal de control en sistemas BCI diseñados para usuarios finales, es decir, para personas con grave discapacidad. La atención selectiva a estímulos específicos e infrecuentes evoca una actividad cerebral específica conocida como P300. Esta respuesta aparece en las zonas central y parietal de la corteza cerebral unos $300 \mathrm{~ms}$ después de la presentación del estímulo. En este estudio, se ha investigado una nueva herramienta BCI asistiva para control del entorno en el hogar. La aplicación propuesta se basa en las respuestas evocadas P300 a estímulos infrecuentes, también conocido como paradigma oddball. Los sistemas BCI basados en P300 podrían ser los más adecuados para las personas con grave discapacidad ya que no requieren de una etapa exhaustiva de entrenamiento. Además, el paradigma P300 típico permite seleccionar el símbolo deseado entre las múltiples opciones presentadas en la pantalla de una manera rápida simplemente fijando la atención en él. La metodología propuesta en este trabajo está enfocada en el usuario final. Así, tanto el diseño, como los experimentos y la evaluación se centran las necesidades del usuario final. 
La población bajo estudio estuvo formada por 30 potenciales usuarios finales de los sistemas BCI. En concreto, en el estudio participaron 30 personas con grave discapacidad, debido a diferentes patologías y con discapacidad no sólo motora sino también cognitiva en algunos casos. Los participantes fueron seleccionados a través del Centro de Referencia Estatal de Discapacidad y Dependencia (León, España). Las principales necesidades identificadas para la población bajo estudio estaban relacionadas con tres categorías: confort, comunicación y seguridad. Así, la herramienta BCI asistiva propuesta se diseñó para cubrir este tipo de necesidades. En concreto, la aplicación permite controlar 8 dispositivos, normalmente presentes en el hogar a través de 115 comandos de control de infrarrojos y 22 comandos de navegación. Para simplificar la interfaz, los comandos se distribuyeron en diez matrices: un menú principal y nueve sub-menús. En la etapa experimental, los sujetos interactuaron con la herramienta propuesta durante tres sesiones, en las que controlaron dispositivos electrónicos reales. Veintitrés de los treinta participantes pudieron controlar adecuadamente la aplicación propuesta, con una precisión superior al 80\%. Diecinueve de ellos incluso obtuvieron una precisión superior al 95\%. Además, los sujetos alcanzaron tasas de transferencia de información de hasta 6.8 item/min y 25.9 bit/min. Estos resultados indican que la mayoría de los potenciales usuarios finales de estos sistemas podrían controlar un sistema BCI basado en potenciales P300 sin necesidad de consideraciones adicionales. Además, el análisis de los resultados sugirió que el grado de discapacidad no suponía un impedimento a la hora de controlar apropiadamente un sistema BCI basado en P300, ya que parecía no influir en el rendimiento de los usuarios manejando la aplicación. De hecho, no se encontró relación entre los resultados de precisión de los participantes y su grado de discapacidad motora, de discapacidad cognitiva o de capacidad de atención sostenida. Por lo tanto, este tipo de aplicaciones podrían ser adecuados para la asistencia y mejora de la autonomía personal en el hogar de las personas con grave discapacidad. Además, la herramienta diseñada podría fácilmente ampliarse y adaptarse a las necesidades específicas de cada paciente. Así, se podría reducir la dependencia que habitualmente presentan estas personas de sus cuidadores, familiares y enfermeros.

La evaluación por parte de los usuarios finales se llevó a cabo mediante cuestionarios de satisfacción. Las respuestas indicaron que los potenciales usuarios finales de los sistemas BCI aceptan de buen grado participar en este tipo de estudios, 
indicando sus necesidades y proporcionando sus puntos de vista. Por ello, las futuras aplicaciones BCI asistivas deberían considerar al usuario final tanto para el diseño, como para su desarrollo y evaluación. La población bajo estudio se mostró satisfecha con la herramienta BCI propuesta según los cuestionarios. La mayoría de los usuarios valoraron positivamente el diseño y utilidad de la aplicación asistiva para un uso habitual en el hogar. En concreto, los usuarios consideraron que la herramienta propuesta era sencilla de manejar y comprender. Además, los participantes indicaron que la aplicación resultaría verdaderamente útil para incrementar su autonomía en su entorno habitual. Los usuarios manifestaron su interés en incluir más dispositivos presentes en el hogar en la aplicación, como persianas, puertas o timbres; sin embargo, no se mostraron especialmente interesados por el uso de sistemas BCI en aplicaciones de movilidad. No obstante, la mayoría de los participantes estuvieron de acuerdo en que el registro de la actividad cerebral mediante electroencefalograma (EEG) no resulta muy práctico para un uso diario. Los usuarios propusieron el uso de gorros inalámbricos y electrodos secos para mejorar este aspecto y conseguir un uso frecuente de la aplicación en su entorno habitual. Estas opiniones de los usuarios finales deberían ser consideradas en futuros estudios de sistemas BCI diseñados para este tipo de poblaciones.

En esta Tesis, se investigó una nueva aplicación BCI asistiva basada en P300 para personas con grave discapacidad. Tanto el diseño, como el procedimiento experimental y la evaluación se llevaron a cabo considerando siempre al usuario final. Además, la etapa experimental se realizó empleando dispositivos reales y tuvo lugar en el entorno habitual de los participantes. Por tanto, este estudio supone un avance importante ya que toma en consideración la opinión y las necesidades de los usuarios finales así como su grado de satisfacción. 



\section{Table of contents}

1. Introduction........................................................................................... 1

1.1. Context: biomedical engineering................................................................. 3

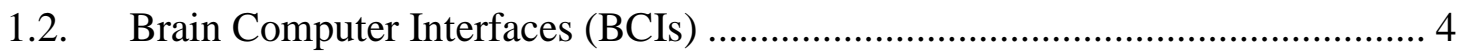

1.2.1. History and definition of BCIs ................................................................ 4

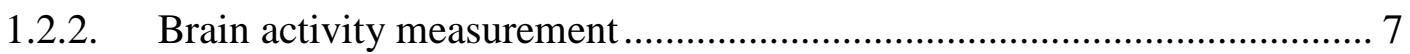

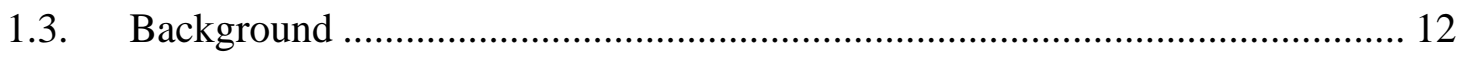

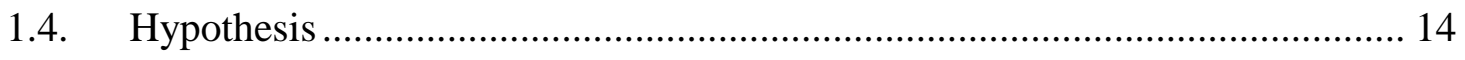

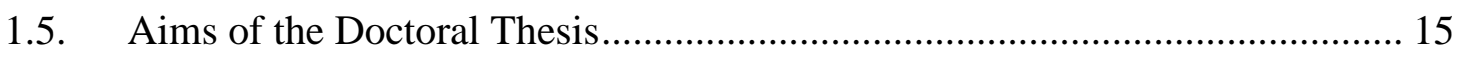

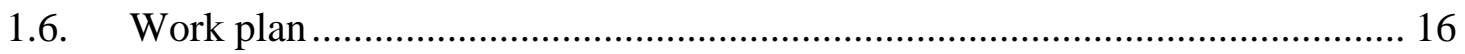

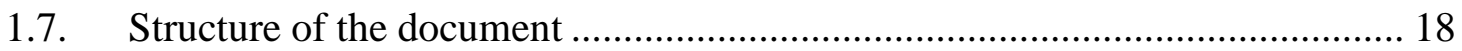

2. State of the art on EEG-based BCls to assist disabled populations.... 21

2.1. Classification of EEG-based BCIs ............................................................... 23

2.1.1. Slow Cortical Potentials (SCPs) ............................................................ 23

2.1.2. Motor imagery or sensorimotor rhythms............................................... 25

2.1.3. Steady-State Visual Evoked Potentials (SSVEPs) .................................. 27

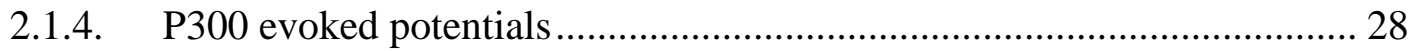

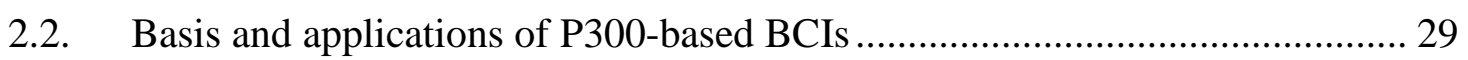

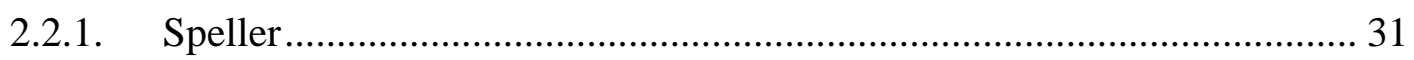

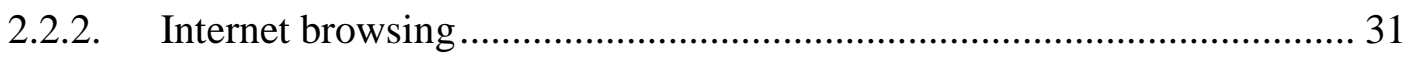

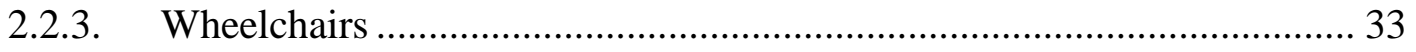

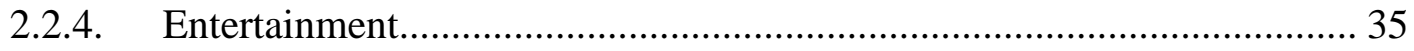

2.3. P300-based BCIs involving disabled populations .......................................... 37

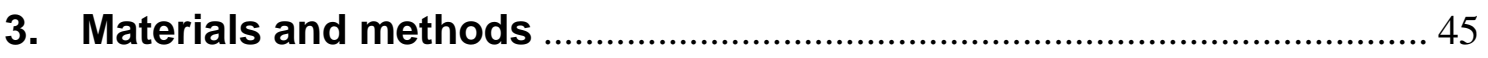

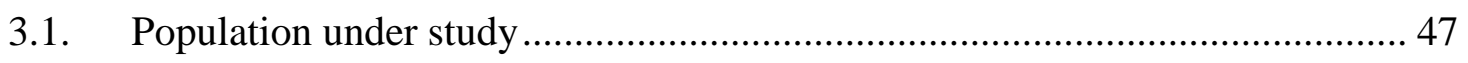

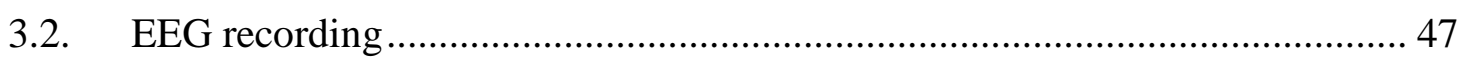

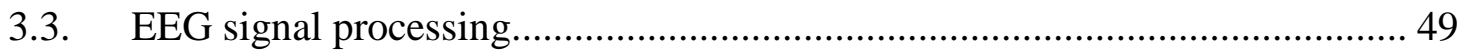

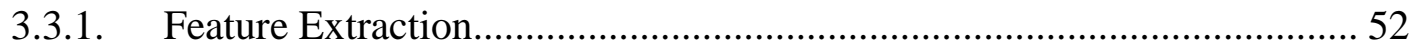

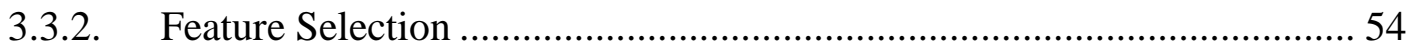

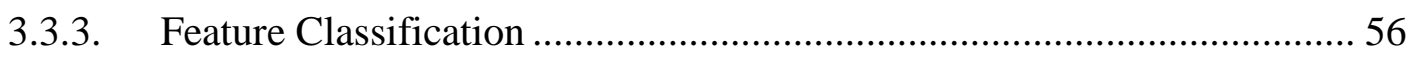




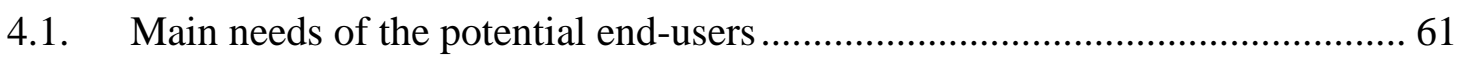

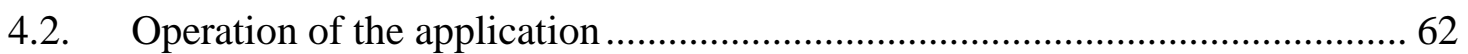

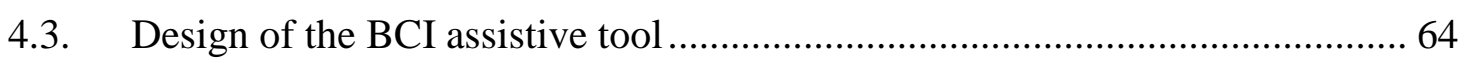

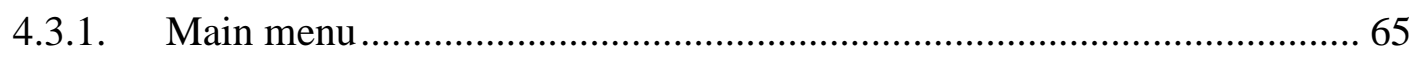

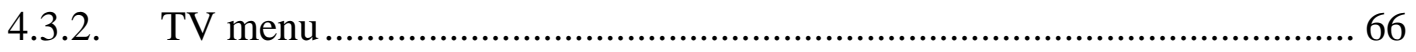

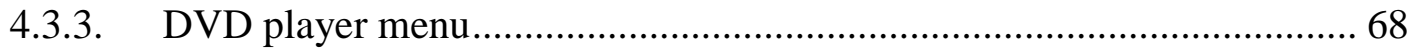

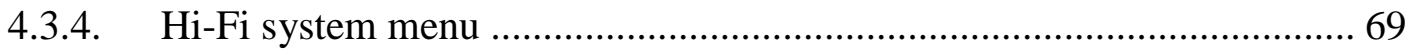

4.3.5. Multimedia hard drive menu ................................................................ 70

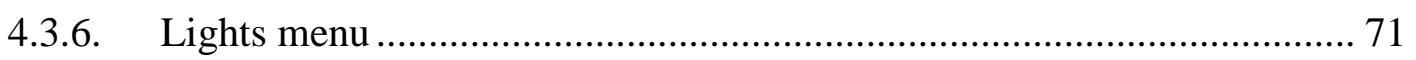

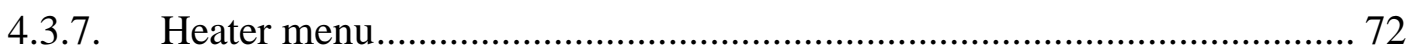

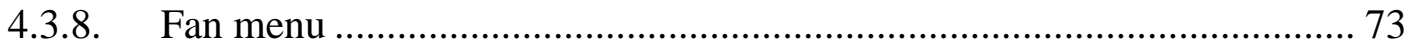

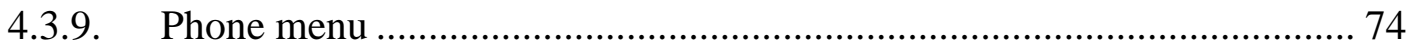

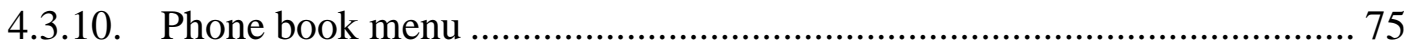

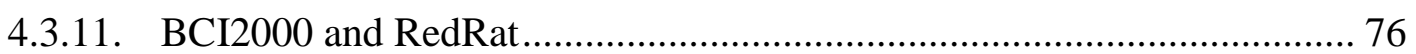

4.4. Assessment procedure by means of real end-users ..................................... 79

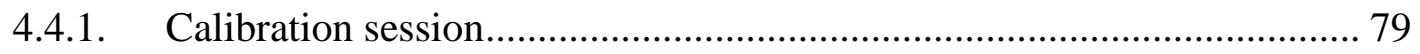

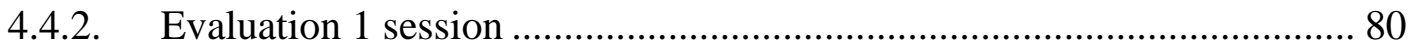

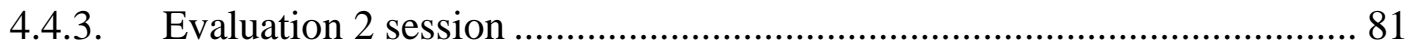

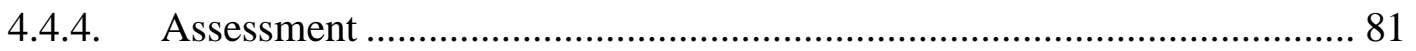

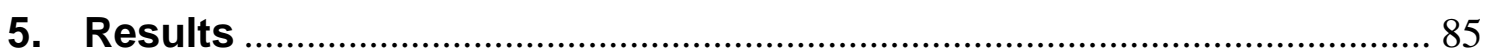

5.1. Performance of end users managing the assistive tool................................ 87

5.2. Assessment of the application by means of the end-users ............................. 89

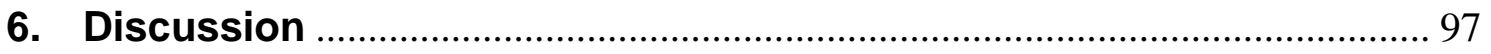

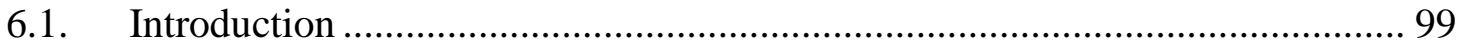

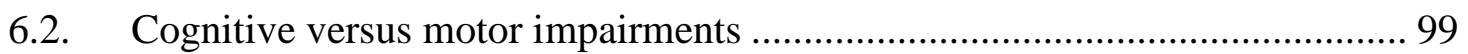

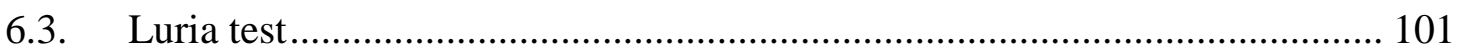

6.4. P300-based studies involving potential end-users....................................... 101

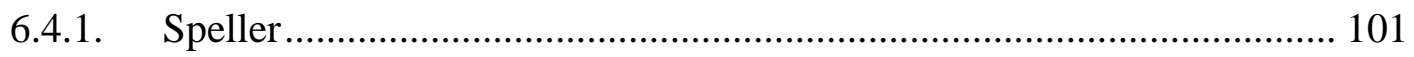

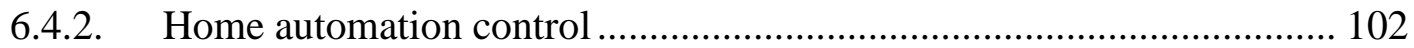

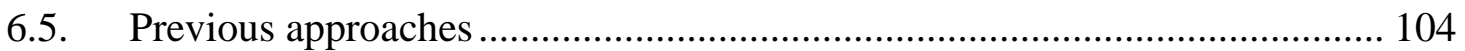

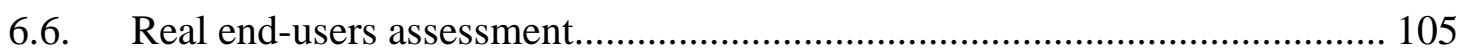

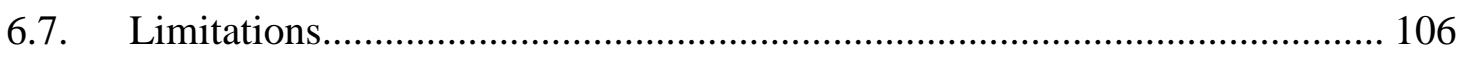




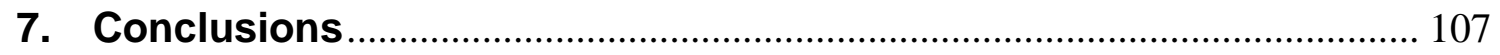

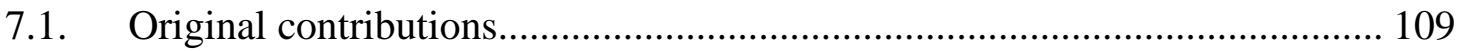

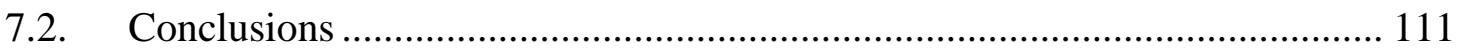

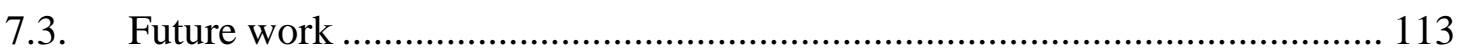

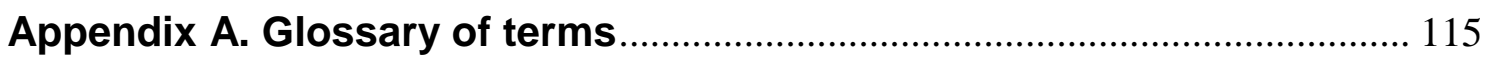

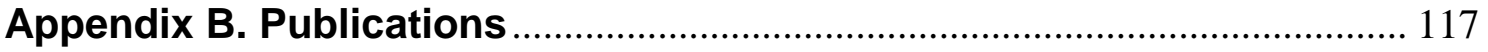

B.1. Peer-Reviewed International Articles in Journals Indexed in the Journal

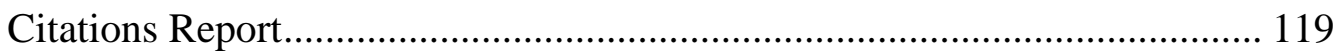

B.2. Peer-Reviewed Articles in not indexed journals ........................................... 119

B.3. Contributions to International Conferences .................................................. 120

B.4. Contributions to National Conferences ......................................................... 122

Appendix C. Síntesis de la tesis doctoral en castellano.............................. 125

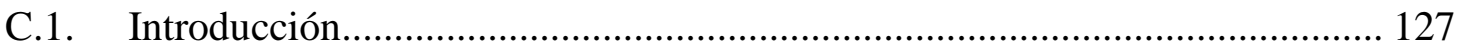

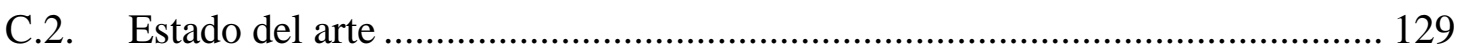

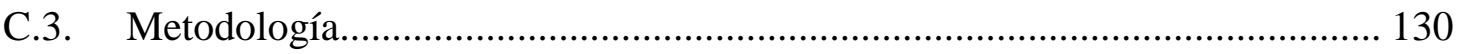

C.4. Aplicación BCI para el control de dispositivos electrónicos presentes en el

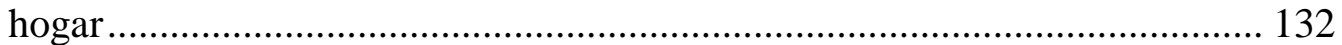

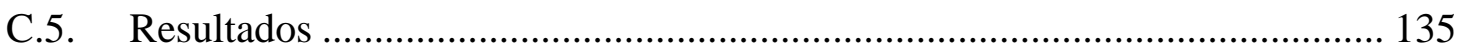

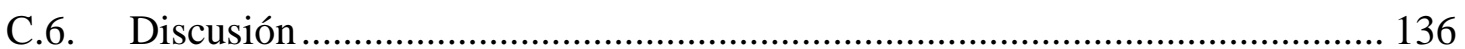

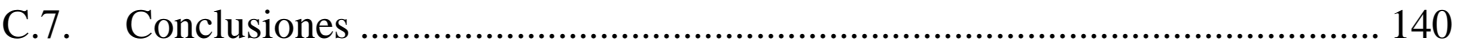

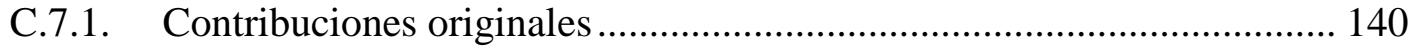

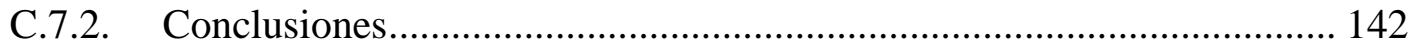

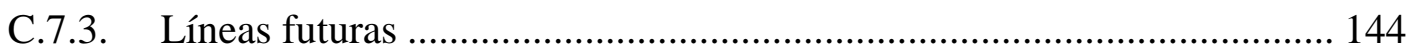

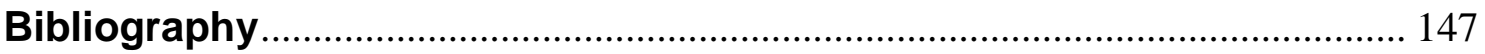





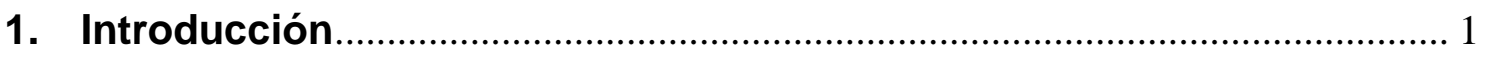

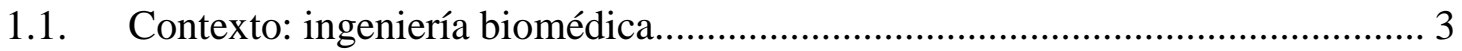

1.2. Interfaces Cerebro-Ordenador (Brain Computer Interfaces, BCI) ..................... 4

1.2.1. Historia y definición de los sistemas BCI ................................................ 4

1.2.2. Registro de la actividad cerebral ........................................................ 7

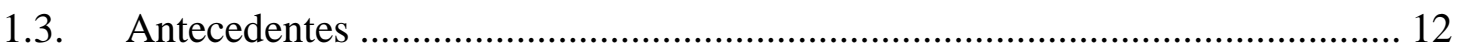

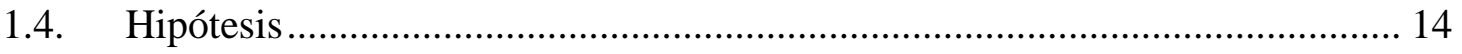

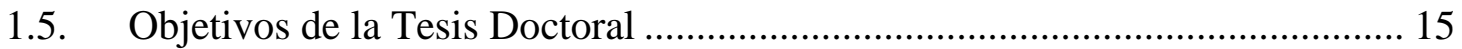

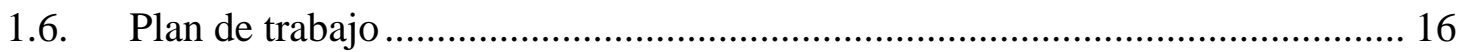

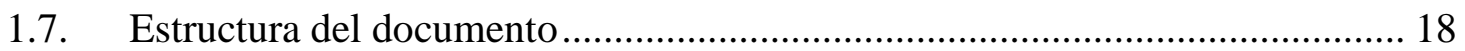

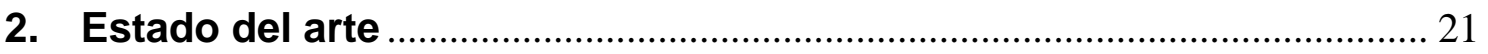

2.1. Clasificación de sistemas BCI basados en EEG............................................... 23

2.1.1. Potenciales corticales lentos ................................................................ 23

2.1.2. Imágenes motoras o ritmos sensoriomotores......................................... 25

2.1.3. Potenciales evocados visuales de estado estable ..................................... 27

2.1.4. Potenciales evocados P300 ................................................................ 28

2.2. Fundamento y aplicaciones de los sistemas BCI basados en P300 .................. 29

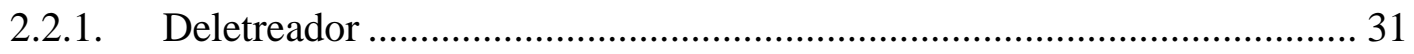

2.2.2. Navegación por Internet ........................................................................ 31

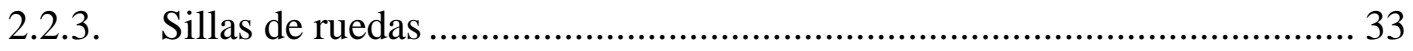

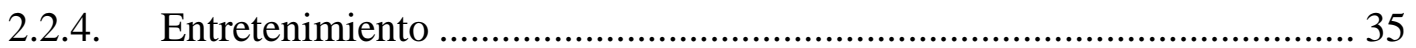

2.3. Sistemas BCI basados en P300 en personas con discapacidad ........................ 37

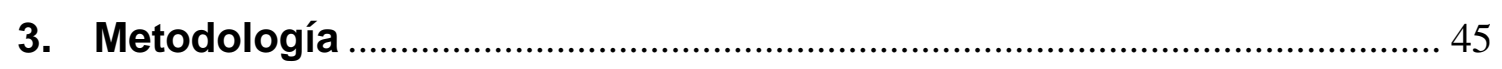

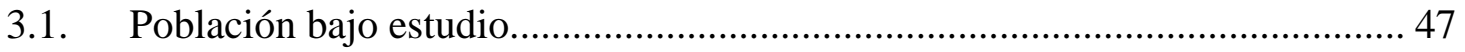

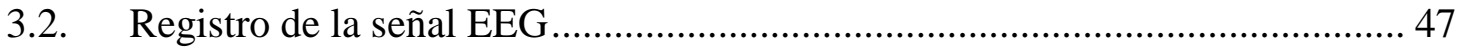

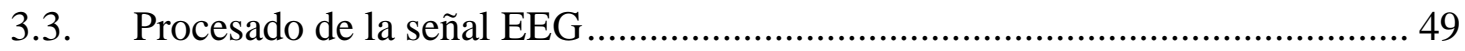

3.3.1. Extracción de características .................................................................. 52

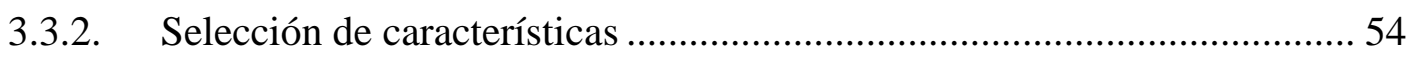

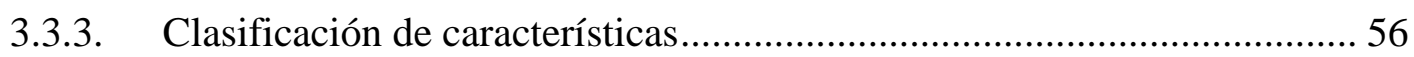




\section{Aplicación $\mathrm{BCl}$ para control de dispositivos electrónicos en el hogar 59}

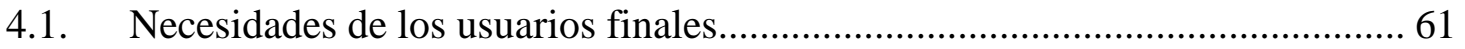

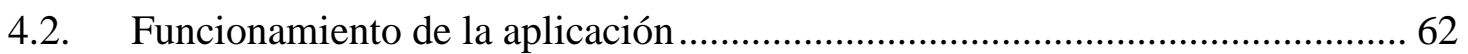

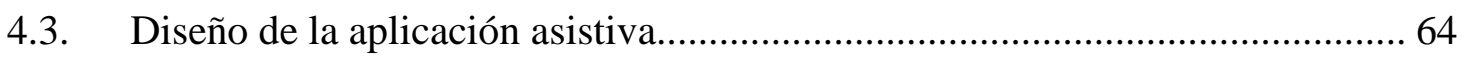

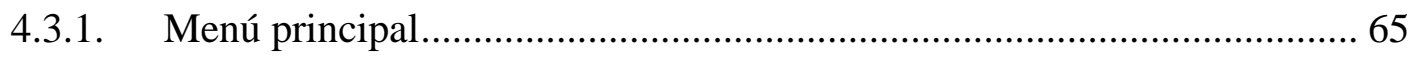

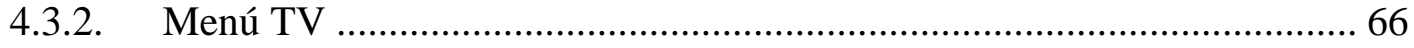

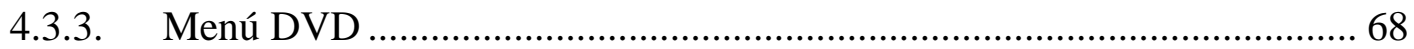

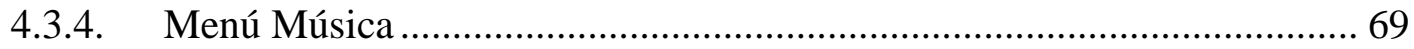

4.3.5. Menú Multimedia ................................................................................. 70

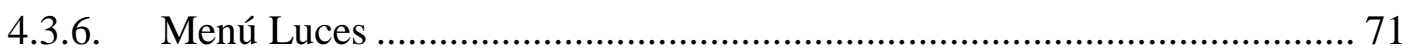

4.3.7. Menú Calefactor ..................................................................................... 72

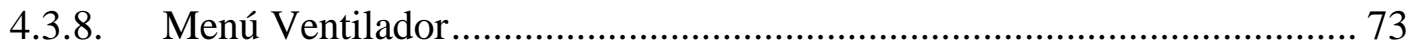

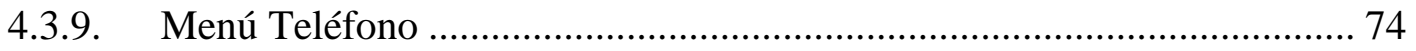

4.3.10. Menú Agenda telefónica........................................................................ 75

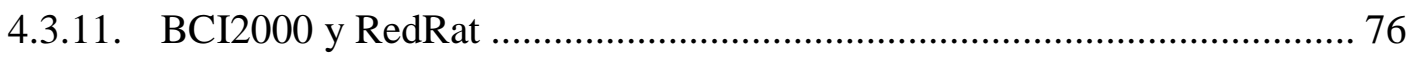

4.4. Procedimiento de evaluación por parte de los usuarios finales......................... 79

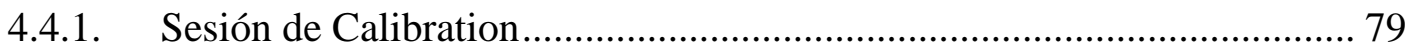

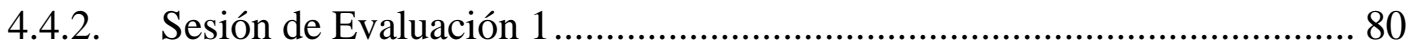

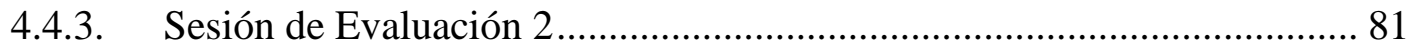

4.4.4. Evaluación de satisfacción...................................................................... 81

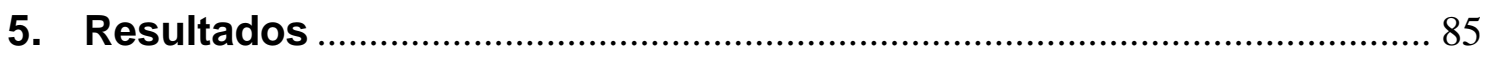

5.1. Rendimiento de los usuarios con la aplicación BCI...................................... 87

5.2. Evaluación de la aplicación por parte de los usuarios finales .......................... 89

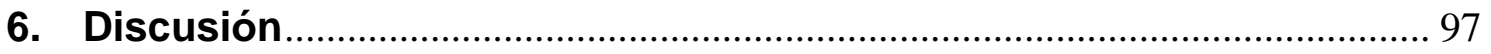

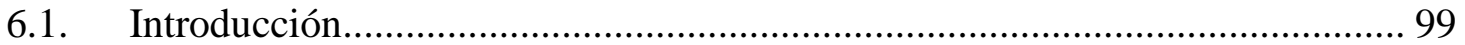

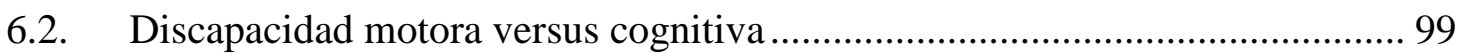

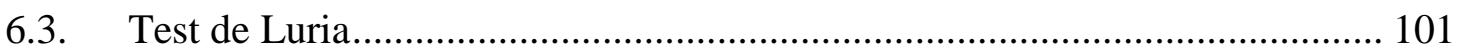

6.4. Estudios basados en P300 aplicados a usaurios finales................................ 101

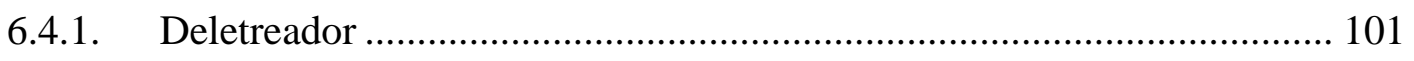

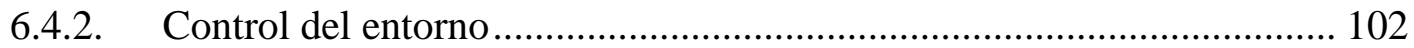

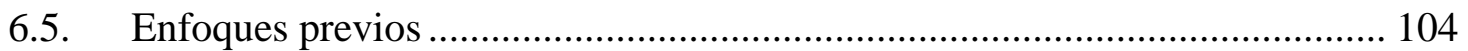

6.6. Evaluación de los usuarios finales................................................................... 105

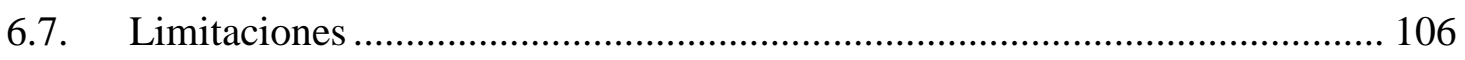




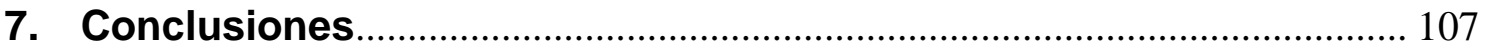

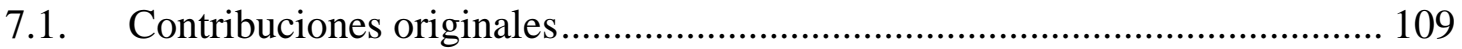

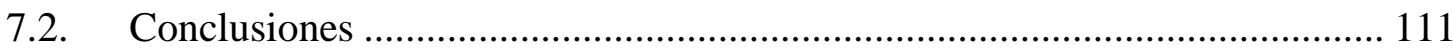

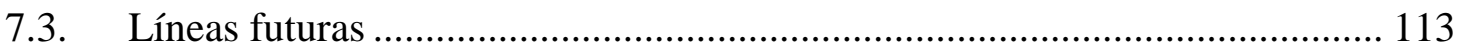

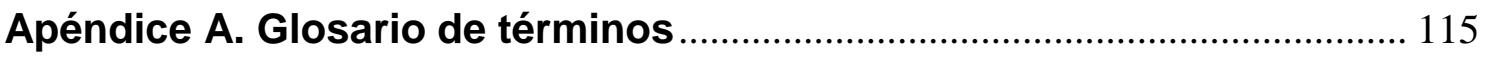

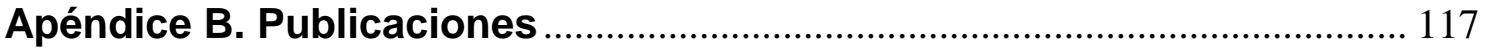

B.1. Artículos revisados por pares en revistas indexadas en el JCR...................... 119

B.2. Artículos revisados por pares en revistas no indexadas ................................ 119

B.3. Contribuciones en Congresos Internacionales ............................................. 120

B.4. Contribuciones en Congresos Nacionales ..................................................... 122

Apéndice C. Síntesis de la tesis doctoral en castellano.............................. 125

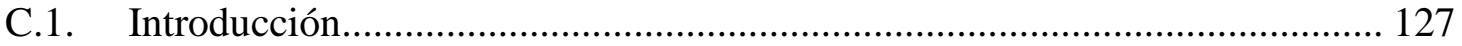

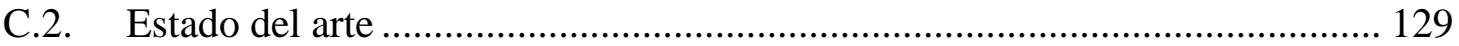

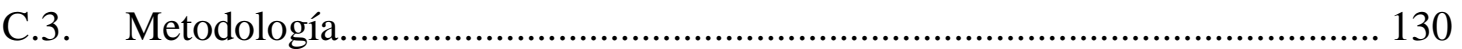

C.4. Aplicación BCI para el control de dispositivos electrónicos presentes en el

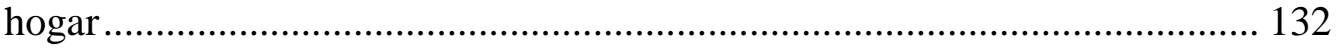

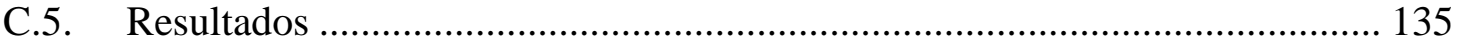

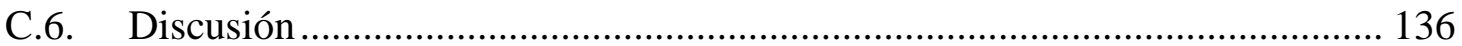

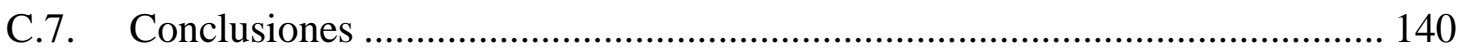

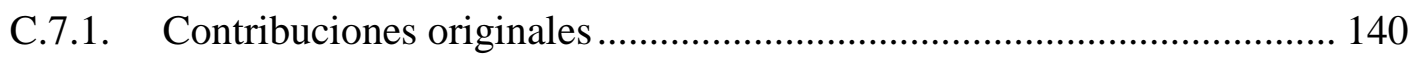

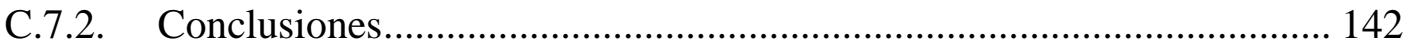

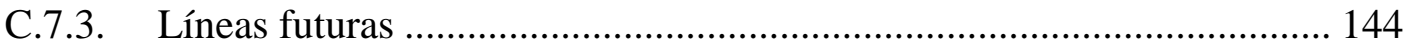

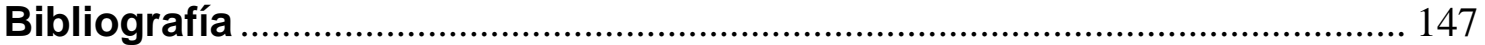





\section{List of Figures}

Figure 1-1. A BCI system bypasses the normal neuromuscular output channels for natural communication and control processes (Graimann et al. 2010).

Figure 1-2. Comparison of different methods for functional brain analysis regarding its spatio-temporal resolution and its degree of invasiveness (Maestú et al. 1999, Poza 2008).

Figure 1-3. Different techniques for recording bioelectrical brain activity (Wolpaw et al. 2006).

Figure 1-4. Typical EEG-based BCI consists of an EEG cap with electrodes, a biosignal amplifier (a device that digitalizes the input brain signals) and a computer. Electrical signals from the scalp are sent to the amplifier and the computer processes the data and translates the brain signals into commands that operate the BCI end devices.

Figure 1-5. The international 10-20 system. (a) View from above the head. (b) View of the left side of the head. The nasion is the intersection of the frontal and nasal bones at the bridge of the nose. The inion is a small bulge on the back of the skull just above the neck.

Figure 2-1. Averaged SCPs of a patient during baseline, baseline interval and feedback interval where cortical positivity was required for selection of a letter and no positivity or negativity was required for rejection of a letter (modified from Birbaumer et al. 2000).

Figure 2-2. Functional regions of the human cerebral cortex. The somatosensory and motor areas are located between the frontal and parietal lobes of the cerebral cortex. 
Figure 2-3. The user can control the amplitude of the $\mu$ rhythm (or the $\beta$ rhythm) in MIbased BCIs in order to move a cursor to a target located at the top or at the bottom of the screen. Frequency spectra show that control is clearly focused in the $\mu$ rhythm frequency band. (Wolpaw et al. 2002).

Figure 2-4. Time course at electrode location $\mathrm{Pz}$ of the voltage for attended (black line) and unattended (grey line) stimuli. The attended stimuli evoke a P300 response waveform.

Figure 2-5. P3 Speller application developed by the BCI2000 team. In this frame the third row is flashed. (Schalk and Mellinger 2010).

Figure 2-6. Stimuli matrix for P300 Browser. In this frame, the second column is flashed. (Mugler et al. 2010).

Figure 2-7. P300-based BCI mouse developed by Citi et al. (2008). The four rectangles at the sides of the screen are associated with the possible movement directions. In this frame, the stimulus of the up direction is presented.

Figure 2-8. (At the top) Picture of a participant navigating with the BCI-controlled wheelchair implemented by Iturrate et al. (2009). (At the bottom) Screenshot of the screen showed to the user for selecting the desired commands.

Figure 2-9. Screenshots from the MindGame (Finke et al. 2009). The player controls the avatar by selecting the target of the next move.

Figure 2-10. Stimuli matrix used in the study of Mak et al. (2012). Groups of items in the speller matrix flashed successively and randomly using the CB paradigm. (Modified from Mak et al. 2012). 
Figure 2-11. Paradigm of the study of Spüler et al. (2012). Top left: rows and columns flash randomly as in the typical speller paradigm. Top right: after classification, a grey square is shown for $1 \mathrm{~s}$. Bottom left: the selected letter is shown to the user for another second. Bottom right: if error-related potentials are detected, the letter is removed and a notification appears. (Spüler et al. 2012).

Figure 2-12. Stimuli matrix used by Hoffman et al. (2008) consisted of 3 x 2 images depicting devices present at home. Images were flashed, one at a time, by changing the overall brightness of images. (Hoffman et al. 2008).

Figure 2-13. Top: Virtual environment used by Aloise et al. (2011b). Users can virtually operate lights, doors, curtains, windows, bed, TV, air condition, alarm and pause the system. Bottom: Picture of a user during the experimental session. The feedback was visually provided as changes in the virtual apartment.

Figure 3-1. EEG recording montage applied in the online sessions.

Figure 3-2. EEG recording equipment used in the present study.

Figure 3-3. P300 evoked potential. (a) Topographical distribution of the P300 response at $330 \mathrm{~ms}$ after stimuli, measured as $\boldsymbol{r}^{2}$ for attended versus unattended items (derived from averaging 15 sequences of stimuli). (b) Time course at $\mathrm{Pz}$ of the voltage for attended (black line) and unattended (grey line) stimuli. (c) Corresponding $\boldsymbol{r}^{2}$ time course that indicates the statistical difference between the attended and unattended items.

Figure 3-4. EEG signal processing comprises three procedures: feature extraction, offline feature selection and online classification. Once the user's desired option is identified, the selected command is sent to the corresponding device.

Figure 3-5. Feature extraction procedure consisted of signal calibration, spatial filtering and temporal filtering stages. 
Figure 3-6. Differences in the time course of $\boldsymbol{r}^{2}$ between not applying any spatial filter and applying CAR to the averaged waveforms. Applied to P300 responses, CAR enhances the statistical difference between the attended and unattended stimuli.

Figure 4-1. (a) Main menu of the assistive BCI tool. It shows a 3 x 4 matrix consisted of images depicting all available devices: TV, DVD player, Hi-Fi system, multimedia drive, lights, heater, fan, and phone. The phone book was included as an independent option in the main menu. Thus, users are able to access from the main menu to a specific sub-menu of each device and to pause, resume or stop the run. (b) Main menu while the third column is dimmed.

Figure 4-2. Picture of the eight real devices that the user can manage by means of the proposed BCI tool.

Figure 4-3. TV menu. It shows a 5 x 5 matrix consisted of images depicting the main TV commands: switch on/off, skip to a specific TV channel, turn up/down or mute the sound, access and navigate TV menu, access the program guide and return to the main menu. From this menu, users are able to operate the TV as using a real remote control.

Figure 4-4. (a) DVD menu. It presents a 3 x 4 matrix consisted of images depicting the main DVD commands: switch on/off, play, pause or stop a video, explore contents, skip to the next or previous file, mute, record the actual channel and return to the main menu. (b) Music menu. It shows a 3 x 4 matrix consisted of images depicting the main musicrelated commands: switch on/off, volume control, skip to radio or CD function, play, pause or stop a track, skip to the next or previous track or radio station and return to the main menu. 
Figure 4-5. (a) Multimedia menu. It shows a 3 x 4 matrix consisted of images depicting the main DVD commands: switch on/off, explore hard drive's contents, play, pause, stop or skip to the next or previous file, show or hide subtitles and return to the main menu. (b) Lights menu. It presents a 3 x 4 matrix consisted of images depicting the main music-related commands: switch on/off, change the light colour, turn up/down the light intensity, enable the flashing mode and return to the main menu.

Figure 4-6. (a) Heater menu. It presents a 3 x 4 matrix consisted of images depicting the main DVD commands: switch on/off, turn up/down power intensity, program the sleep function from 30 min to 4 hours, enable or disable the swing mode and return to the main menu. (b) Fan menu. It shows a 3 x 4 matrix consisted of images depicting the main music-related commands: switch on/off, increase or decrease speed, program the sleep function, enable or disable a specific internal fan or the swing mode and return to the main menu.

Figure 4-7. Phone menu. It shows a $4 \times 4$ matrix consisted of images depicting the main phone control commands: pick up or hang up the phone, dial a phone number, automatically dial the emergency phone number, redial the last phone number, go to the phone book menu for dialling a previously memorized phone number and return to the main menu.

Figure 4-8. Phone book menu. It presents a 3 x 4 matrix consisted of images depicting the main phone book commands: nine names of people for automatically dial, which phone numbers were previously memorized, automatically dial the emergency phone number, hang up the phone and return to the main menu.

Figure 4-9. IR signal database created for managing electronic devices at home (bd_devices.xml). The database is divided into 8 devices (TV, DVD player, heater, air conditioning, Hi-Fi system, multimedia drive, lights and phone), which contain their specifics commands. In the picture the commands list used for lights control is expanded. 
Figure 4-10. Picture of a participant during one of the experimental sessions with the BCI tool.

Figure 4-11. Assessment procedure for this study. Each subject was intended to perform three sessions: one session comprised of calibration tasks and two evaluation sessions for assessing their performance with the designed BCI tool (Evaluation1 and Evaluation 2).

Figure 5-1. Results of the first group of participants managing the assistive BCI tool. Online accuracies (ACC) for Calibration, Eval1 and Eval2 sessions are shown.

Figure 5-2. Results of the second group of participants managing the assistive BCI tool. Online accuracies (ACC) for Calibration, Eval1 and Eval2 sessions are shown.

Figure 5-3. Topographical distribution of P300 response (first column), averaged response according to the stimulus type (second column), and $\boldsymbol{r}^{2}$ waveform (third column) for: (a) participant P2, who only performed Calibration tasks once; (b) subject P26, who carried out Calibration tasks twice; (c) and user P13, who performed Calibration tasks during three sessions. Usually, the more prominent the P300 amplitude peak is, the more accuracy is reached by the user.

Figure 5-4. Pictures of two participants using the assistive BCI tool to manage different electronic devices: (a) TV, (b) Fan.

Figure 5-5. Graphical distribution of responses given by the participants to each statement of the assessment questionnaire. Odd statements were positive assertions about the proposed BCI tool (ideal response: $e$ ), whereas even statements were negative assertions (ideal response: $a$ ). 
Figure 5-6. Averages and standard deviations of the scores assigned by the participants to each statement of the questionnaire. Odd statements were positive assertions about the proposed BCI tool (ideal score: 4), whereas even statements were negative assertions (ideal score: 0). 



\section{List of tables}

Table 2-1. Summary of different studies for novel P300-based applications. Number of healthy subjects, main details of each work and the mean online accuracy (ACC) are shown.

Table 2-2. Comparison of current studies involving P300-based spellers and disabled participants. Number of impaired subjects, main details of each study, mean online accuracy (ACC) and maximum information transfer rate (ITR) are provided.

* Four additional subjects were not included since they did not complete the study.

Table 2-3. Comparison of different studies involving P300-based applications for environment control and disabled participants. Number of impaired subjects, main details of each study, mean online accuracy (ACC) and maximum information transfer rate (ITR) are shown.

* One additional subject was not included since classification accuracies above chance level could not be obtained.

${ }^{* *}$ Only offline classification results are provided. Four-fold cross-validation was used to estimate the accuracy.

Table 3-1. Demographic and clinical data of the first group of participants in the study. Sex: male (M), female (F).

Motor/Cognitive impairment degree: absent (A), mild (m), moderate (M), severe (S), profound (P). Sustained attention ability: very good (VG), good $(G)$, moderate $(M)$, poor (P), very poor (VP).

Table 3-2. Demographic and clinical data of the second group of participants in the study.

Sex: male (M), female (F).

Motor/Cognitive impairment degree: absent (A), mild (m), moderate (M), severe (S), profound (P). Sustained attention ability: very good (VG), good (G), moderate (M), poor (P), very poor (VP). 
Table 4-1. The most common requirements of potential BCIs end-users from the NRCD\&D.

Table 4-2. Assessment questionnaire of the proposed assistive BCI tool (translated from Spanish). All participants completed the questionnaire after having finished all experimental sessions.

Table 5-1. Results for each participant managing the assistive BCI tool. Online accuracy (ACC) for each kind of session, maximum item selection rate $(S R)$ with the pause between selections (and without the pause between selections in parentheses), and maximum information transfer rate (ITR) with the pause between selections (and without the pause between selections in parentheses).

${ }^{*}$ : During the third session these participants repeated Eval1 tasks, due to accuracy lower than $70 \%$ during the previous Eval1 session.

${ }^{* *}$ : During the second session this participant repeated Calibration tasks, due to accuracy lower than $70 \%$ during the previous Calibration session. During the third session, this participant performed Eval1 tasks.

${ }^{* * *}$ : During the second and third sessions this participant repeated Calibration tasks , due to accuracy lower than 70\% during the previous Calibration sessions.

Table 5-2. Questionnaire results for the assessment of the assistive BCI tool.

Each statement was rated on a 5-point Likert scale (0-strongly disagree, 1-disagree, 2-neutral, 3agree, 4-strongly agree). Odd statements were positive assertions about the assistive BCI tool (ideal score: 4) while even statements were negative assertions (ideal score: 0). 
Table 6-1. Comparison of different studies involving P300-based BCIs and severely disabled participants. Number of impaired subjects, main details of each paradigm and application studied, number of performed sessions and number of total item selections (trials) across all sessions, number of sequences of stimuli, mean online accuracy (ACC) and maximum information transfer rate (ITR) without pause between selections are provided.

* Four additional subjects were not included since they did not complete the study or algorithms failed to detect reliably the evoked potentials.

** One additional subject was not included since classification accuracies above chance level could not be obtained.

*** Only offline classification results are provided. Four-fold cross-validation was used to estimate the accuracy. 



\section{Introduction}

1.1. Context: biomedical engineering

1.2. Brain Computer Interfaces (BCIs)

1.2.1. History and definition of BCIs

1.2.2. Brain activity measurement

1.3. Background

1.4. Hypothesis

1.5. Aims of the Doctoral Thesis

1.6. Work plan

1.7. Structure of the document 



\subsection{Context: biomedical engineering}

The present study falls into the biomedical engineering domain, which is defined as the development and application of engineering principles and techniques to effectively provide solutions to problems in biology and medicine (Nebeker 2002), as well as to monitor physiological functions and assist in patient diagnosis and treatment (Bronzino 2006). This multidisciplinary field involves knowledge of medicine, biology, physics, mathematics or engineering and covers a wide scope of areas such as biomedical signal and image processing, biomechanics, prosthetic devices and artificial organs, biomaterials, biotechnology, tissue engineering, neural engineering, biomedical instrumentation, physiological modelling, bionanotechology, rehabilitation engineering, bioinformatics and genomics, clinical engineering or biosensors (Enderle et al. 2005). This Doctoral Thesis is focused on biomedical signal processing and neural engineering areas.

Biomedical signals are those which are produced by biological structures and systems (Cohen 2006). Thus, there is a wide variety of biomedical signals. Attending to their origin, signals can be classified into different groups such as bioelectrical signals (i.e., electroencephalogram, electrocardiogram, electromyogram and electrooculogram, electroenterogram, electrogastrogram y electroretinogram); biomagnetic signals (i.e., magnetoencephalogram and magnetocardiogram); biochemical signals (i.e., partial pressures of oxygen and carbon dioxide); biomechanical signals (i.e., mechanomyogram and aerial flux pressure); bioacoustic signals (i.e., phonocardiogram and ecography); biooptical signals (i.e., blood oxygen saturation); etc. Moreover, biomedical signals can also be divided according to their field of application (i.e., neurology, cardiology, etc.) and their characteristics (i.e., continuous and discrete, stationary and non-stationary signals, etc.) (Cohen 2006). Typically, physicians analysed biomedical signals by visual inspection. Signal processing allows to automate the measurements of signal properties, resulting in reduced subjectivity and more reliability (Martí 1988). It is also really useful for uncovering components with low amplitude and/or subtle variations in frequency that are very difficult to observe visually. In addition, it enables to remove undesired components in order to enhance the signal-to-noise ratio and to facilitate the analysis (Laguna and Sörnmo 2009). 
The biomedical signal analysed in this Doctoral Thesis is the electroencephalogram (EEG). Electroencephalography consists on the recording of the spontaneous brain electrical activity along the scalp. It is a portable technique and it requires relatively simple and inexpensive equipment (Wolpaw et al. 2002). Since the EEG was first described in 1929 by Hans Berger (Berger 1929), it has been mainly used to evaluate neurological disorders in the clinic, to investigate brain function in the laboratory and to explore its therapeutic possibilities (Wolpaw et al. 2002). Over this time, people have speculated that the EEG might be used to decipher thoughts or intents (Wolpaw et al. 2002). Thus, EEG could be used for communication and control and enable the brain to act on the environment without the normal intermediaries of peripheral nerves and muscles (Wolpaw et al. 2000). Therefore, a Brain Computer Interface (BCI) is a communication system that monitors brain activity and translates specific signal features, reflecting the user's intent, into commands that operate a device (Wolpaw et al. 2002). This definition reflects the principal reason for recent interest in BCI development: it offers a new augmentative communication technology to those who are paralyzed or have other severe movement deficits. Other augmentative communication technologies require some form of muscle control, and thus may not be useful for those with the most severe motor disabilities, such as late-stage amyotrophic lateral sclerosis (ALS), brainstem stroke or severe cerebral palsy (Wolpaw et al. 2000). The study presented in this Doctoral Thesis is focused on the application of EEG-based BCIs to assist severely impaired individuals at their own home.

\subsection{Brain Computer Interfaces (BCIs)}

\subsubsection{History and definition of BCIs}

The first BCI system was implemented in 1964 by Dr. Grey Walter (Graimann et al. 2010). He connected electrodes directly to the motor areas of a patient's brain, which was undergoing surgery for other reasons. The individual was asked to press a button to advance a slide projector while Dr. Walter recorded the brain activity. Afterwards, he connected the system to the projector so that the slide projector advanced whenever the patient's brain activity indicated that he wanted to press the button. Not only the system detected properly when the patient wanted to press the 
button, but also Dr. Walter found that it was necessary to introduce a delay from the detection of the brain activity until the slide projector advanced, since the slide projector would otherwise advance before the patient pressed the button (Graimann et al. 2010). That was the first BCI system: it was possible to detect control intention before the actual movement. Unfortunately, Dr. Walter did not publish this major discovery; he only described it in a talk to a group called the Ostler Society in London (Dennett 1992, Graimann et al. 2010). Afterwards, in 1977, Dr. Jacques Vidal used computer-generated visual stimulation and signal processing, showing that visual evoked potentials (VEPs) could provide a communication channel for controlling the movement of a cursor through two dimensions (Vidal 1977, Wolpaw et al. 2000). There was little progress in BCI research for a long time since then. BCI research advanced slowly for many more years. At the turn of the century, there were only one or two dozen groups doing serious BCI research (Graimann et al. 2010). Nevertheless, BCI research has advanced quickly during the last years: there are more BCI-related papers, contributions to conferences, talks, and products every year (Fazel-Rezai 2011, Guger et al. 2013, Guger et al. 2014a, Guger et al. 2014b). Nowadays, there are more than one hundred BCI research labs operative and this number is growing (Graimann et al. 2010).

Natural procedures of communication or control require the intervention of peripheral nerves and muscles. The user's intent initiates a complex process in which specific brain areas are activated and hence signals are sent through the peripheral nervous system to the corresponding muscles, which in turn execute the required movement for the desired task (Graimann et al. 2010). A BCI system provides an alternative to natural communication and control procedures. A BCI is an artificial system that bypasses the body's normal efferent pathways, which are the neuromuscular output channels (Wolpaw et al. 2002), as shown in Figure 1-1. Instead of depending on peripheral nerves and muscles, a BCI directly monitors brain activity related to the user's intent and translates the recorded brain activity into control signals for managing a device.

A BCI system must satisfy four requirements. First, it must record brain activity directly from the brain (invasively or non-invasively). Second, it must provide feedback to the user. Third, this feedback must be available in real-time. Finally, the system must rely on intentional control. In other words, the user must decide to perform a mental task whenever he wants to reach a goal with the BCI. Accordingly, the devices 


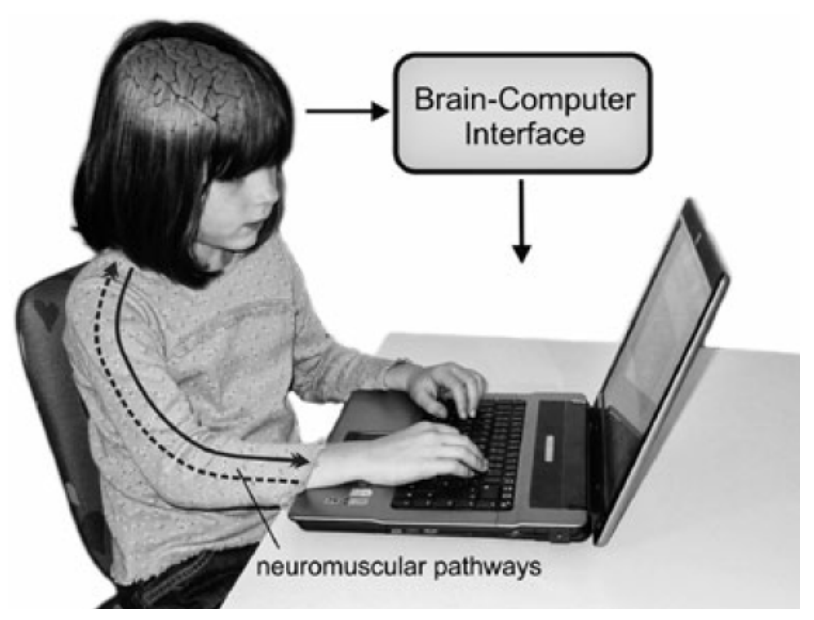

Figure 1-1. A BCI system bypasses the normal neuromuscular output channels for natural communication and control processes (Graimann et al. 2010).

that passively detect changes in brain activity that occur without any intent, such as activity related to workload, arousal, sleep, etc. are not BCIs (Graimann et al. 2010).

Most researchers accept the term 'BCI' and its definition. However, other terms have been used to define this special form of human-machine interfaces, such as brain-machine interfaces (BMIs) (Donoghue 2002), direct brain-interfaces (DBIs) (Levine et al. 1999) or neuroprostheses (Schwartz 2004). There is no difference between BCI, BMI and DBI. They exactly describe the same system and they can be used as synonyms. Nevertheless, 'neuroprosthesis', also called neural prosthesis, is a device that not only receives output from the nervous system but can also provide input. Cochlear implants (auditory neural prostheses) or retinal implants (visual neural prostheses) are examples of neuroprostheses. BCIs are a special subcategory of neuroprostheses that provides an artificial output channel from the central nervous system (Graimann et al. 2010).

As it was previously remarked, BCIs are human-computer interfaces that offer direct artificial output channels from the brain and thus do not require muscle activity. This is significant since BCIs can provide assistive tools for severely disabled individuals that cannot perform physical movements. Nowadays, some diseases can really lead to the locked-in syndrome (Nijboer and Broermann 2010). In this condition, the person is cognitively intact but the body is completely paralyzed, i.e. any voluntary control of muscles is lost. Patients cannot move their legs, arms or faces and depend on artificial respirators. The active and completely functional mind is confined in the body. ALS is a disease that can lead to this syndrome. Its exact cause is unknown and there is 
no cure for patients. ALS starts with muscle weakness and atrophy. Then, voluntary movements, such as walking, speaking, swallowing and breathing, deteriorate over several years. Finally, muscular control is lost entirely. However, the cognitive functions are not affected by the disease. Patients can still see, hear and understand what happens around, but cannot control their muscles. The reason is that ALS only affects special neurons, the large alpha motor neurons, which are an integral part of the motor pathways (Graimann et al. 2010). Death is usually caused by failure of the respiratory muscles. Artificial respiration and nutrition can considerably prolong the life expectancy but this leads to life in the locked- in state. Once the motor pathway is lost, any natural way of communication with the environment is lost as well. The only effective way to communicate is using a device that can read brain signals and convert them into control signals. Therefore, BCIs provide the only option for communication in such cases (Birbaumer and Sauseng 2010).

\subsubsection{Brain activity measurement}

BCIs monitor and record brain activity, process it, and generate control signals that reflect the user's intent (Wolpaw et al. 2002). The brain activity generates electrical and magnetic changes (Graimann et al. 2010). Hence, sensors can detect this electrical or magnetic activity at different times over different areas of the brain in order to study the brain activity. These changes can be measured by means of invasive or noninvasive sensors. In this section, both types of recording techniques will be described. Figure 1-2 shows a diagram with different methods for functional brain analysis regarding its temporal and spatial resolution and the degree of invasiveness. In this regard, different technologies can be used for the study of brain activity (Maestú et al. 1999):

- Methods that use the radiation from particles o radionuclides: single photon emission computed tomography (SPECT) and positron emission tomography (PET).

- Methods that record signals from electromagnetic sources:

o Methods that use external electromagnetic sources in order to generate an image: functional magnetic resonance imaging 


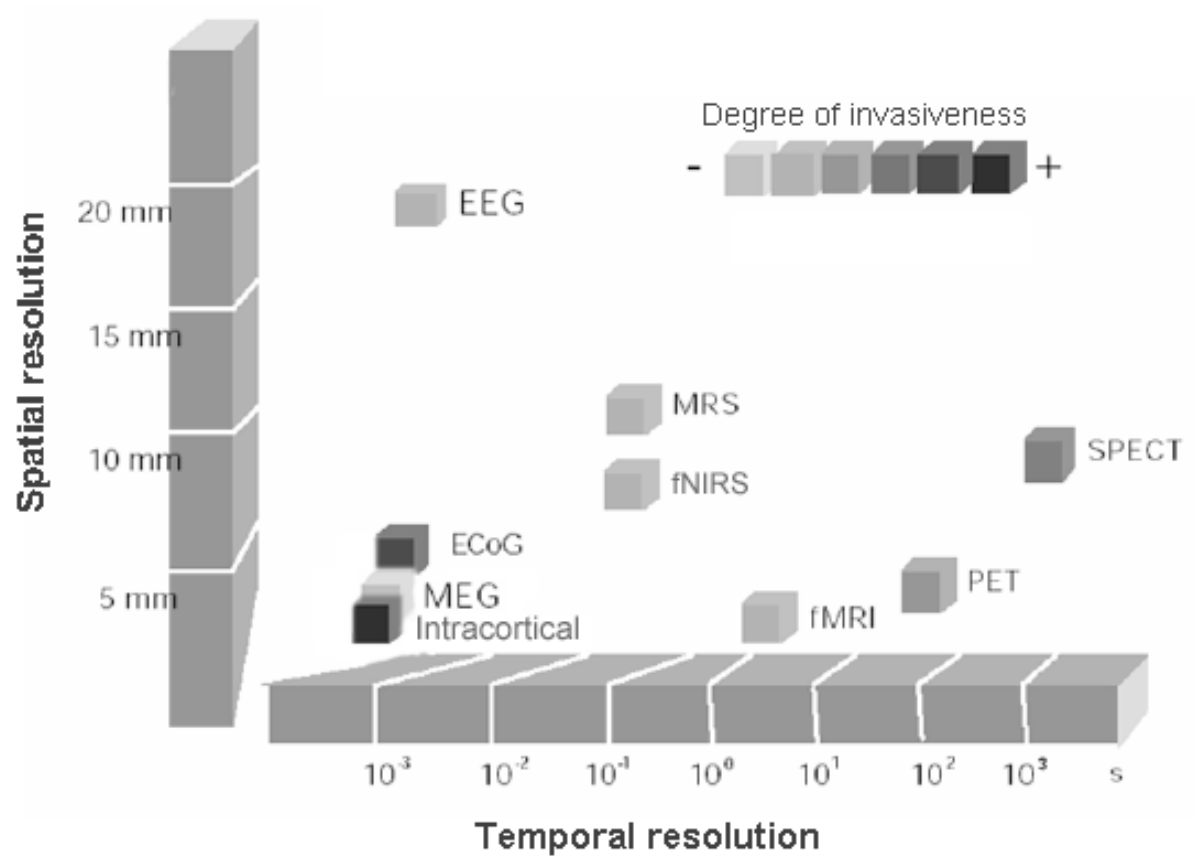

Figure 1-2. Comparison of different methods for functional brain analysis regarding its spatio-temporal resolution and its degree of invasiveness (Maestú et al. 1999, Poza 2008).

(fMRI), functional near-infrared resonance spectroscopy (fNIRS) and magnetic resonance spectroscopy (MRS).

o Methods that record electromagnetic brain activity originated by the brain: electroencephalography (EEG), electrocorticography (ECoG), magnetoencephalography (MEG), and intracortical recordings.

PET and SPECT both present several limitations. Firstly, it is not possible to repeat the studies indefinitely since although patients are exposed to low doses of radiation, these ones have cumulative effect (Eliassen et al. 2008, Poza 2008). Secondly, both techniques have very low temporal resolution, limited by the sensitivity of the radiation detector, and SPECT also presents low spatial resolution. Finally, both methods require expensive equipment especially PET, which also demands high maintenance costs.

fMRI and MRS subject the patient under intensive magnetic fields (from 20 to 80000 times the magnetic field of the Earth), which effects are not clearly defined. Moreover, MRS has medium spatio-temporal resolution and the technique is still under development, while fMRI presents high spatial resolution but low temporal resolution 
(Poza 2008). fNIRS has higher temporal resolution and besides it is less invasive and expensive than fMRI. However, it presents poorer spatial resolution and the cost of the required equipment is still high (Bauernfeind et al. 2008, Cui et al. 2011, Dornhege et al. 2007).

The techniques that record electromagnetic activity generated by the brain are the only methods that measure directly the neural activity instead of recording it as a function of the metabolism or the blood flood. Furthermore, they usually have the highest temporal resolution and, in the case of ECoG, intracortical recordings and MEG they also present high spatial resolution.

MEG maps brain activity by recording magnetic fields generated by electrical currents occurring naturally in the brain. To that purpose, MEG uses very sensitive magnetometers such as superconducting quantum interference devices (SQUIDs). MEG offers both high spatial and temporal resolution. Moreover, it presents high sensitivity for recording the brain signal, since magnetic fields are not distorted through the tissues because it has the same magnetic permeability as the air (Poza 2008). However, MEG requires large and expensive equipment which makes it not portable and thus impractical for usual BCI applications.

Nowadays, the most common BCIs use bioelectrical brain activity. The electrical brain activity can be measured by means of invasive or non-invasive techniques. Figure 1-3 summarizes the different methods for recording the bioelectrical brain activity: ECoG, intracortical recordings and EEG.

Invasive recording techniques require surgery in order to implant the necessary sensors. This surgery involves opening the skull through a craniotomy and cutting the membranes that cover the brain. Two kind of signals can be measured. Firstly, the signal recorded from electrodes placed on the surface of the cortex, which is called the electrocorticogram (ECoG). ECoG cannot damage any neurons since electrodes do not penetrate the brain (Miller and Ojemann 2010). Secondly, the signal recorded from electrodes that penetrate brain tissue, which is called intracortical recording (Graimann et al. 2010, Taylor and Stetner 2010).

On one hand, invasive recording methods provide excellent signal quality, very good spatial resolution and a higher frequency range. Moreover, artefacts are less problematic with invasive recordings. Intracortical electrodes can record the neural 


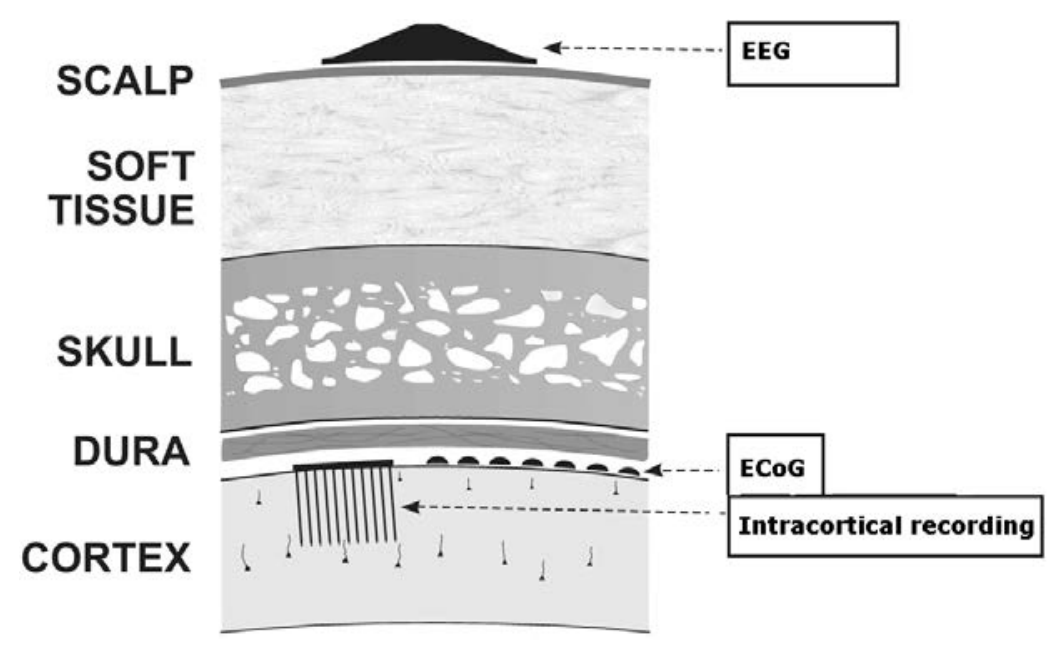

Figure 1-3. Different techniques for recording bioelectrical brain activity (Wolpaw et al. 2006).

activity of a single neuron or small assemblies of neurons; whereas the ECoG records the integrated activity of a much larger number of brain cells that are in the proximity of the ECoG electrodes. Furthermore, any invasive recording method has better spatial resolution than the EEG (Graimann et al. 2010). On the other hand, although invasive techniques have some advantages over non-invasive ones, the main drawback of invasive methods is the required surgery. Financial, ethical and other considerations make neurosurgery impractical except for patients who need a BCI to communicate. Even then, some of these subjects may find that a non-invasive BCI fulfils their needs. Moreover, it is unclear whether both intracortical recordings and ECoG could provide safe and stable recordings over years, especially in the case of intracortical recordings. Electrodes implanted into the cortex can cause tissue reactions that lead to deteriorated signal quality or even complete electrode failure (Graimann et al. 2010). Since neurosurgery is expensive and risky, research on invasive BCIs is problematic. Some invasive research efforts rely on patients who undergo surgery for other causes, such as treatment of epilepsy. These studies can be very revealing, but it is not possible to investigate the effects of training and long-term use because these subjects usually have the invasive recording system for only a few days before it is removed (Graimann et al. 2010).

Non-invasive recording methods do not require surgery or even break the skin. Most BCIs work with electrical measures of brain activity and rely on sensors placed over the head to measure this activity. EEG records electrical activity from electrodes located over the scalp. It is a very well-known technique, which has been 
used in research and clinical contexts for decades. EEG is a portable and easy to apply technique that requires relatively simple and inexpensive equipment (Wolpaw et al. 2002). Moreover, EEG presents a very good temporal resolution. Nevertheless, EEG also has drawbacks. The spatial resolution and the frequency range are limited. EEG is susceptible to present artefacts or contaminations due to other electrical activities. Bioelectrical activities caused by eye movements or blinks, electrooculographic activity (EOG), and by muscles, electromyographic activity (EMG), close to the recording locations as well as external electromagnetic sources, such as the power line, can easily contaminate the EEG (Graimann et al. 2010). Figure 1-4 presents a typical EEG-based BCI.

As presented above, there are several methods to record the brain activity. The method most commonly used for BCI systems is the EEG. Although it has low spatial resolution, it presents important advantages over other methods: noninvasiveness, low cost, portability, and simplicity. These features make EEG more practical for most BCI applications than the remaining recording techniques. Despite EEG is not very technically demanding, the setup process can be difficult to deal with. The skin that is contacted by the electrodes has to be carefully prepared with special

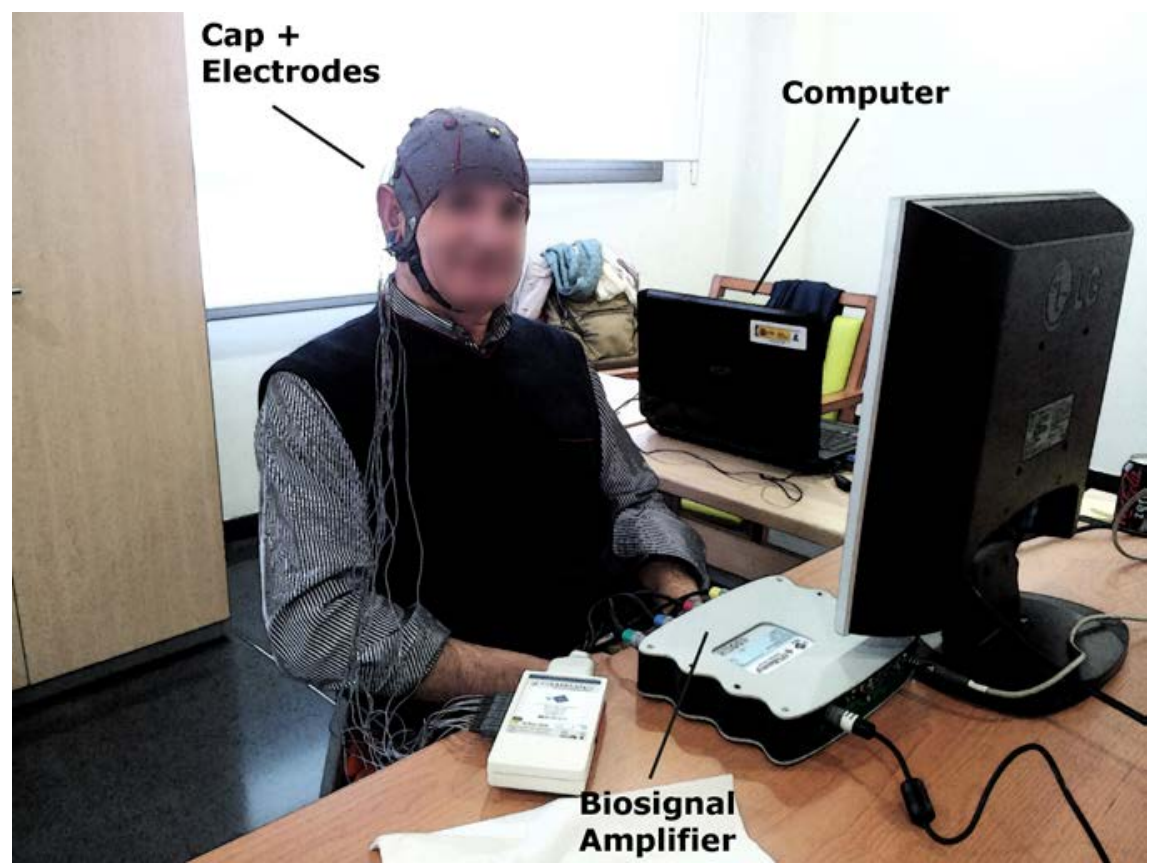

Figure 1-4. Typical EEG-based BCI consists of an EEG cap with electrodes, a biosignal amplifier (a device that digitalizes the input brain signals) and a computer. Electrical signals from the scalp are sent to the amplifier and the computer processes the data and translates the brain signals into commands that operate the BCI end devices. 
abrasive gel in order to achieve suitable signal quality. Gel is the reason because these electrodes are usually known as wet electrodes. The number of necessary electrodes in an EEG-based BCI depends on the own specific system and ranges from a few to more than 200 electrodes. Typically, BCI researchers address their efforts to minimize the number of electrodes in order to reduce the setup time and annoyance. The setup procedure has to be repeated before each BCI sessions because gel dries out and wearing the EEG cap with the electrodes and cables is not comfortable or fashionable. Possible solutions based on dry electrodes, which do not require electrode gel, and on wireless electrodes are currently being researched. However, a practical solution that provides signal quality comparable to wet electrodes is not ready at the moment.

A BCI system monitors the brain activity looking for patterns originated from specific brain areas. To obtain consistent recordings from specific areas of the head, scientists trust on a standard system for placing the electrodes accurately. This system is called the International 10-20 System (Jasper 1958, Niedermeyer and Silva 2005). It is widely used in clinical EEG recording and EEG research as well as BCI research. The name 10-20 indicates that the most commonly used electrodes are placed $10,20,20,20,20$, and $10 \%$ of the total nasion-inion distance. The other electrodes are positioned at similar fractional distances. The inter-electrode distances are equal along any transverse (from left to right) and antero-posterior (from front to back) line and the placement is symmetrical (Graimann et al. 2010). The electrode positions are marked with a label. The first letters of the labels indicates the brain area over which the electrode is located: Fp: pre-frontal, F: frontal, C: central, P: parietal, O: occipital and T: temporal. The number or the letter ' $z$ ' in the label indicates the transversal position and the distance to the central line. Figure 1-5 illustrates the electrode placement according to the international 10-20 system.

\subsection{Background}

A few years ago the Biomedical Engineering Group of the University of Valladolid started a new research line focused on BCIs in order to apply its knowledge of advance biomedical signal processing. Our main objective was to develop applications for real BCI end-users, i.e., people with severe motor impairments. Since 


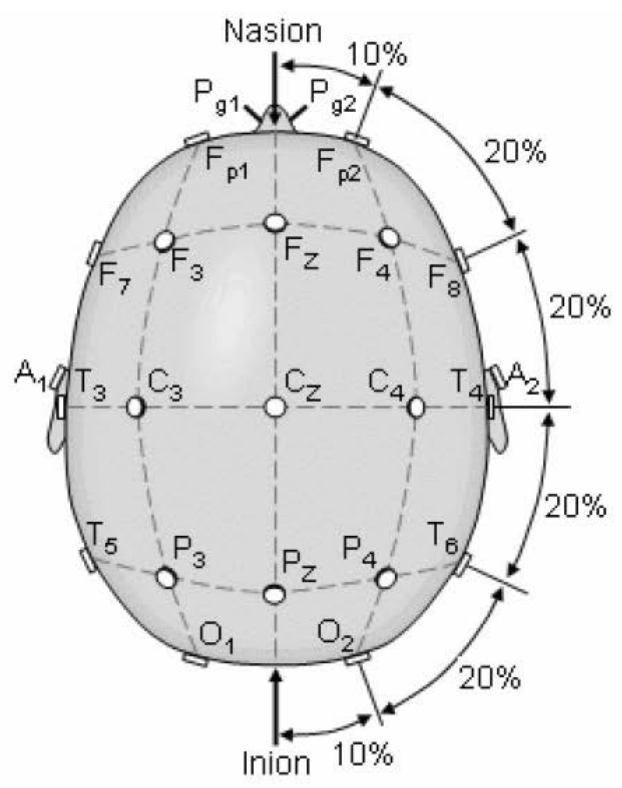

(a)

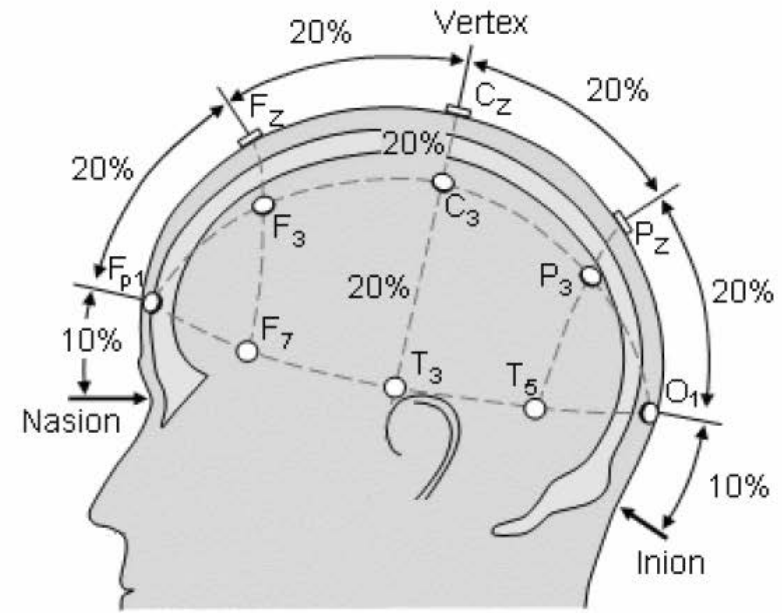

(b)

Figure 1-5. The international 10-20 system. (a) View from above the head. (b) View of the left side of the head. The nasion is the intersection of the frontal and nasal bones at the bridge of the nose. The inion is a small bulge on the back of the skull just above the neck.

there were no actual BCI applications for home automation control, we decided to focus on this scope. To that purpose, we got in contact with the Spanish NRC-D\&D, or National Reference Centre on Disability and Dependence (Centro de Referencia Estatal de Discapacidad y Dependencia), and they supported our idea of increasing the autonomy and independence at home of their users with a BCI application.

Our first approach to BCIs for home automation control was by means of motor imagery (MI). The movement of a limb or even the contraction of a single muscle changes the brain activity in the cortex. Actually, the preparation or the imagination of a movement without actually performing the movement produces brain activity patterns that can be voluntarily controlled (Graimann et al. 2010, Pineda et al. 2000). MI-based BCIs depend on the user's ability to control this internal electrophysiological activity in order to move a cursor in the screen (Wolpaw et al. 2000). The number of devices and functionalities controlled by the proposed application were limited due to the nature of MI-based BCIs. Specifically, it allowed managing 4 electronic devices (TV, DVD player, Hi-Fi system and lights) and up to 19 control commands. The preliminary results in our lab were promising with healthy users. Nevertheless, the users from the NRC$\mathrm{D} \& \mathrm{D}$, who presented not only motor disabilities but also cognitive ones, had severe 
difficulties to achieve high accuracy moving the cursor to the desired targets. MI-based BCIs require an extensive training period and a high degree of concentration ability to move the cursor. It is quite probable that the cognitive impairments affected their attention ability and thus their performance. To overcome this limitation, the present Doctoral Thesis studies the use of P300 evoked potentials to control a BCI tool for managing electronic devices at home. P300-based BCIs would not require extensive training since the brain spontaneously reacts to an external stimulus. Furthermore, this paradigm would allow to present the users more simultaneous choices for each selection.

Our initial studies also brought up the electrodes issue. The set-up of EEG passive electrodes can take too much time. We noticed that this usually had a negative influence on the end-user's motivation later. To avoid this issue, this Doctoral Thesis proposes the use of EEG active electrodes instead of passive ones. Therefore, the set-up time should be significantly reduced and users' motivation should be increased.

\subsection{Hypothesis}

BCIs translate specific features from the brain activity into commands that operate any device. These systems are especially interesting for people with severe disabilities since they could increase their autonomy and thus being more independent from caregivers, relatives, nurses, etc. Although severely impaired individuals would be the typical end-users of BCIs, most of the studies related to BCI systems usually involve healthy people (Mak et al. 2011). In P300-based BCIs, the response detection is typically accomplished using traditional classifiers that discriminate between signals that contain the P300 evoked response and ones that do not (Schalk 2006). For healthy individuals, the discriminant function can generalize to other datasets from the same subject (Schalk 2006). However, for disabled populations the P300 response can be more variable and the discriminant function may not generalize well to other datasets (Schalk 2006). There are a limited number of studies involving BCIs and severely impaired subjects. Nevertheless, due to the nature of these studies, they are usually assessed with a small disabled population. 
In this Doctoral Thesis, it is hypothesised that severely impaired individuals can suitably control P300-based BCIs for managing assistive applications. It is proposed to research an assistive BCI for home automation control and to assess whether a large population of severely disabled people is able to properly manage it. Individuals will manage real home devices in their usual environment and they will also assess the design, interface, usability and usefulness of the proposed tool for their daily life. Although impaired users might not be able to set up the system without aid of others, this Thesis takes a significant step to assess the degree to which such populations could effectively manage a stand-alone system.

\subsection{Aims of the Doctoral Thesis}

The main aim of this Thesis is to research whether P300 evoked potentials are suitable to severely impaired people for managing real BCI applications. The novelty of the study lies in two aspects. Firstly, the use of an environment control application in a real scenario: real devices, usually present at home, managed by endusers in their own environment. Secondly, the application assessment through a large population of severely impaired people. The performance of subjects managing the application is measured as online accuracies and information transfer rates. In addition, the possible influence that the degree of motor and cognitive disability of each individual could have on the BCI performance is investigated. Each individual is also requested for assessing the design and usefulness of the proposed tool for regular use a home. In order to achieve these major objectives, the following specific objectives must be fulfilled:

1. To recruit a large population of individuals with severe impairments.

2. To research into the state of the art of P300-based BCIs and their application to disabled populations.

3. To analyse the main needs of disabled people at home and to design the assistive tool according to them.

4. To develop the assistive BCI tool for managing electronic devices at home by means of P300 responses. 
5. To design the assessment methodology of the experiment.

6. To carry out the experimental procedure with the recruited population.

7. To create a database of EEG recordings corresponding to subjects performing the assessment tasks with the proposed BCI tool.

8. To quantify the performance of each patient by measuring accuracies and information transfer rates.

9. To interpret the obtained results and investigate whether the degree of disability could influence the users’ performance.

10. To publish the main findings of the research in international journals with a relevant impact factor.

\subsection{Work plan}

In order to achieve the previously proposed objectives, the following work plan was designed:

1. Recruitment of a large population of people with severe disabilities. The population involved in this Thesis is composed of subjects, BCI-naives, with motor impairments. Some of them also presented cognitive disability. Subjects were patients from the Spanish NRC-D\&D (National Reference Centre on Disability and Dependence), located in San Andrés del Rabanedo (León, Spain). All the people gave their consent to participate in the study.

2. Bibliographic search about P300-based BCIs. An exhaustive analysis of the latest studies about the application of P300-based BCIs was performed. Studies involving disabled population were especially considered. Different signal processing methods for controlling P300-based BCIs were also studied and those methods that could better identify the user's intent were selected.

3. Analysis of the main needs of potential end-users with collaboration of the patients and staff (physiotherapists, neuropsychologists, nurses, assistants, etc.) from the NRC-D\&D. 
4. Design of the assistive BCI tool combining the requirements of P300-based BCI paradigm and the main identified needs of real end-users.

5. Implementation of the selected signal processing methods in MATLAB ${ }^{\circledR}$.

6. Development of the proposed application for environment control. Software was implemented using $\mathrm{C}++$ programming language with the Borland $\mathrm{C}++$ Builder 6 development environment. It must be noted that some of the required programs are included in the BCI2000 general-purpose system developed by the BCI2000 team (Schalk and Mellinger 2010).

7. Design of the assessment procedure for the study. The experimental assessment comprised three sessions: one initial session for calibrate/adjust the parameters for the next sessions and two evaluation sessions for managing the proposed assistive tool. Finally, a questionnaire was provided to the participants in order to evaluate the BCI tool from their point of view.

8. Execution of the experimental phase. Each subject performed three sessions with the proposed BCI tool and their EEG signals were stored. The equipment used was a g.USBamp biosignal amplifier (Guger Technologies OG, Graz, Austria). Recordings were referenced to the right earlobe and grounded to channel Fpz, respectively. Data was recorded at a sample frequency of $256 \mathrm{~Hz}$.

9. Development of a database of EEG recordings. The recordings corresponding to each participant during each experimental session were stored together with their age, gender and other clinical information, such as the degree of motor and cognitive impairment, in the database.

10. Measurement of the performance of each patient. Online accuracies and information transfer rates achieved by the participants during the experimental session were calculated.

11. Interpreting the results, discuss them and drawing conclusions. The results reached by the end-users were interpreted. It was also investigated whether the degree of impairment could affect the users' performance with the BCI tool. The main conclusions from these results were drawn. Finally, the results of the Thesis were compared with those reported in other studies. 
12. Publication of the results. Some preliminary results of the Thesis were published in international journals of the biomedical engineering field. Moreover, national and international conferences in this field were considered. Additional relevant findings will be considered for future publication.

\subsection{Structure of the document}

The main concepts, hypothesis, aims and methodology of this Thesis were introduced in the present section. At this point, the organisation and contents of the document are described. It is divided into seven chapters, including the present one that serves as introduction. The content of each chapter is detailed below.

- Chapter 2. State of the art on P300-based BCIs. A detailed description of these specific BCIs is included. Then, an exhaustive analysis of other proposed applications and studies with disabled populations is detailed.

- Chapter 3. Material and methods. Firstly, this chapter describes the population under study. Secondly, the acquisition process of EEG signals is explained. Finally, EEG signal processing methods are described.

- Chapter 4. BCI tool for managing electronic devices at home. The fourth chapter deals with the design and development of the proposed assistive tool. The main requirements of the potential end-users are identified. Then, the basis of the operation for the proposed application and its implementation through different submenus are described. Finally, the evaluation procedure by means of a disabled population is detailed.

- Chapter 5. Results. The results achieved in this research are presented in this chapter. Firstly, the performance reached by the participants operating the assistive BCI tool is reported. Secondly, the patients' assessment of the proposed application from the point of view of daily use at home is summarized. 
- Chapter 6. Discussion. The results presented in the previous chapter are exhaustively analysed. Additionally, a comparison with other previous studies is carried out. Finally, the limitations of this study are noted.

- Chapter 7. Conclusions. The main findings of the present study are highlighted. Moreover, the main contributions of the Thesis as well as future work for this study are identified. 



\section{State of the art on EEG-based BCls to assist disabled populations}

\subsection{Classification of EEG-based BCIs}

2.1.1. Slow Cortical Potentials (SCPs)

2.1.2. Motor imagery or sensorimotor rhythms

2.1.3. Steady-State Visual Evoked Potentials (SSVEPs)

2.1.4. P300 evoked potentials

2.2. Basis and applications of P300-based BCIs

2.2.1. Speller

2.2.2. Internet browsing

2.2.3. Wheelchairs

2.2.4. Entertainment

2.3. P300-based BCIs involving disabled populations 



\subsection{Classification of EEG-based BCIs}

EEG is commonly used to record brain activity in non-invasive BCIs because it is a portable technique that requires relatively simple and inexpensive equipment (Corralejo et al. 2014, Wolpaw et al. 2000). Successfully recording of the brain activity is the first step for BCIs. Nevertheless, recording this activity is not enough since a BCI does not read the mind or thoughts in general. A BCI only detects and classifies specific patterns of activity in the current brain signals that are associated with specific tasks or events (Graimann et al. 2010). According to the nature of these input signals, EEG-based BCIs can be classified into two groups. Endogenous BCIs depend on user control of electrophysiological activity originated internally, such as amplitude in a specific frequency band in EEG recorded over a specific cortical area (Wolpaw et al. 2000). BCIs based on motor imagery or slow cortical potentials (SCPs) are endogenous systems and often require extensive training. Endogenous BCI's provide a better fit to a control model since the trained user exercises direct control over the environment (Wolpaw et al. 2000). On the other hand, exogenous BCIs depend on electrophysiological activity originated externally, i.e., evoked by specific stimuli. They may not require extensive training but they require a slightly structured environment. BCIs based on P300 potentials or steady-state visual evoked potentials (SSVEPs) are exogenous systems (Corralejo et al. 2014, Wolpaw et al. 2002). The main EEG-based approaches previously mentioned are described below.

\subsubsection{Slow Cortical Potentials (SCPs)}

Among the lowest frequency features of the EEG signal there are slow voltage changes generated in cortex. These potential shifts occur over 0.5-10.0 s and are called slow cortical potentials (SCPs). Negative SCPs are usually related to movement and other functions involving cortical activation, while positive SCPs are typically associated with reduced cortical activation (Birbaumer 1997, Rockstroh et al. 1989). Figure 2-1 illustrates SCPs typical waveforms. Individuals can learn to control SCPs and thus control the movement of a cursor on a computer screen (Birbaumer et al. 2000, Wolpaw et al. 2002). SCPs have been tested extensively in people with late-stage ALS and have proved to be able to supply basic communication capability (Kübler et al. 1999, Kübler et al. 2001). 
The typical paradigm of SCPs-based BCIs shows the user a computer screen that presents one option at the top and other one at the bottom. SCPs are extracted by appropriate EEG filtering, corrected for EOG artefacts and use to provide visual feedback to the user. One selection takes about $4 \mathrm{~s}$. In the first $2 \mathrm{~s}$, the system measures the initial voltage level. In the subsequent $2 \mathrm{~s}$, the user voluntarily decreases or increases the baseline voltage level in order to select the top or bottom choice in the screen. The voltage is displayed as vertical movement of a cursor which indicates the current selection. Auditory or tactile feedback can also be provided (Birbaumer et al. 2000). SCPs-based BCIs require previous training. Commonly, users train for several sessions of approximately 1-2 h over weeks or months until they reach accuracies above $75 \%$ (Birbaumer et al. 2000). Then, they can use SCPs to select letters by a series of twochoice selections (Perelmouter and Birbaumer 2000). The first two selections choose between the two halves of the alphabet, the next two between the two quarters of the selected half, and so on until a single letter is chosen. Users who have two-choice accuracies of $65-90 \%$ can write $0.15-3.0$ letters/min or 2- 36 words/h (Wolpaw et al. 2002). Although these rates are low, these systems are still useful for people who cannot use other augmentative communication technologies. Moreover, communications rates could be considerably increased by means of predictive algorithms. Finally, SCPs based-BCIs can be also used by disabled users to navigate through the Internet (Birbaumer et al. 2000).

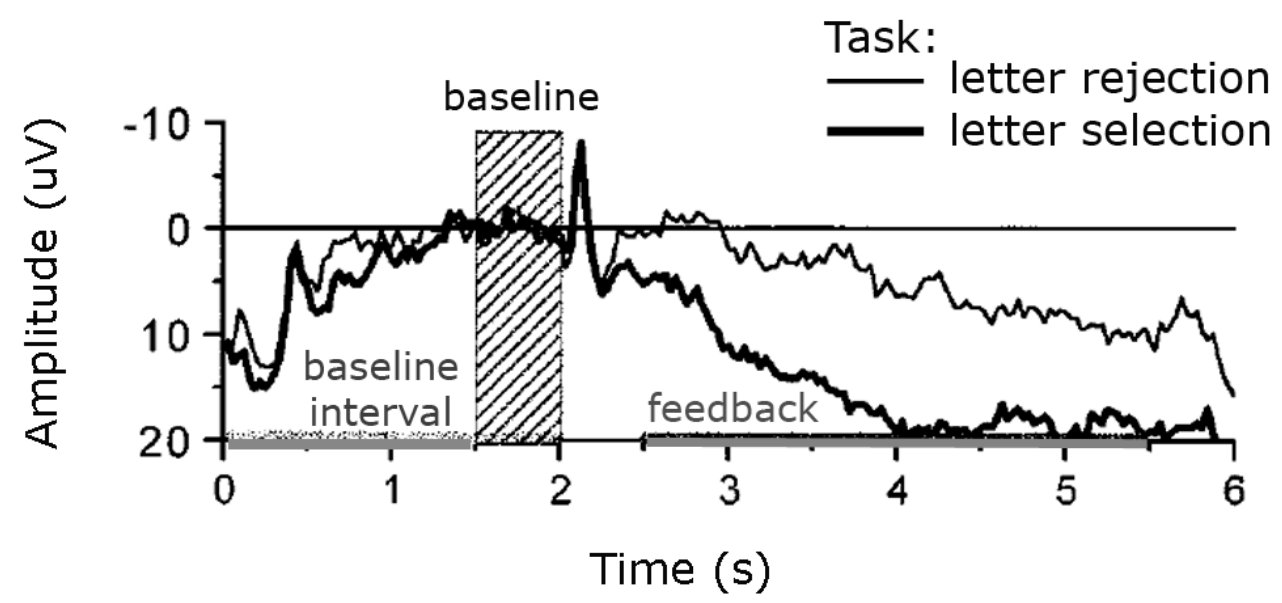

Figure 2-1. Averaged SCPs of a patient during baseline, baseline interval and feedback interval where cortical positivity was required for selection of a letter and no positivity or negativity was required for rejection of a letter (modified from Birbaumer et al. 2000). 
SCPs-based BCIs are slow. Hence, the reached information rates are poor. These rates could improve if users are able to produce more than two SCP levels at the same location or control SCPs at several locations independently but this implies very well-trained users (Wolpaw et al. 2002).

\subsubsection{Motor imagery or sensorimotor rhythms}

The movement of a limb or even the contraction of a single muscle changes the brain activity in the cortex. In fact, the preparation of a movement or the imagination of a movement also changes the brain activity and thus the called sensorimotor rhythms (SMR). SMR refer to oscillations in the brain activity recorded from somatosensory and motor cortical areas (see Figure 2-2). The oscillations of the brain activity are usually divided according to specific frequency bands: $\delta$ ( $<4 \mathrm{~Hz}$ ), $\theta$ (4-7 Hz), $\alpha$ (8-12 Hz), $\beta$ (12-30 Hz) and $\gamma$ (> $30 \mathrm{~Hz}$ ) (Graimann et al. 2010). Typically, the alpha band activity recorded from sensorimotor areas is known as $\mu$ rhythm. The decrease of oscillatory activity in a specific frequency band is called event-related desynchronization (ERD). Similarly, the increase of oscillatory activity in a specific frequency band is called event-related synchronization (ERS). The imagination of movement without actually performing the movement produces ERD/ERS patterns that can be voluntarily generated (Graimann et al. 2010, Pineda et al. 2000). For MI-based BCIs, the most important frequency bands are $\mu$ mu and $\beta$ (Wolpaw et al. 2002).

The invoked brain activity by right hand movement imagery is most prominent over electrode C3, located in the left hemisphere (see Figure 1-5). Analogously, left hand movement imagery generates brain activity most prominent over $\mathrm{C} 4$, located in the right hemisphere. Consequently, the brain activity produced by hand movement imagery is located on the contralateral side (Graimann et al. 2010). Foot movement imagery generates activity over electrode $\mathrm{Cz}$ but a distinction between left and right foot movement is not possible using EEG because the corresponding cortical areas are too close. Similarly, ERD/ERS patterns of individual fingers cannot be discriminated using EEG (Graimann et al. 2010). The cortical areas corresponding to hand, foot and tongue are comparatively large and topographically different compared to the remaining EEG (EEG background activity) to produce patterns that can be 


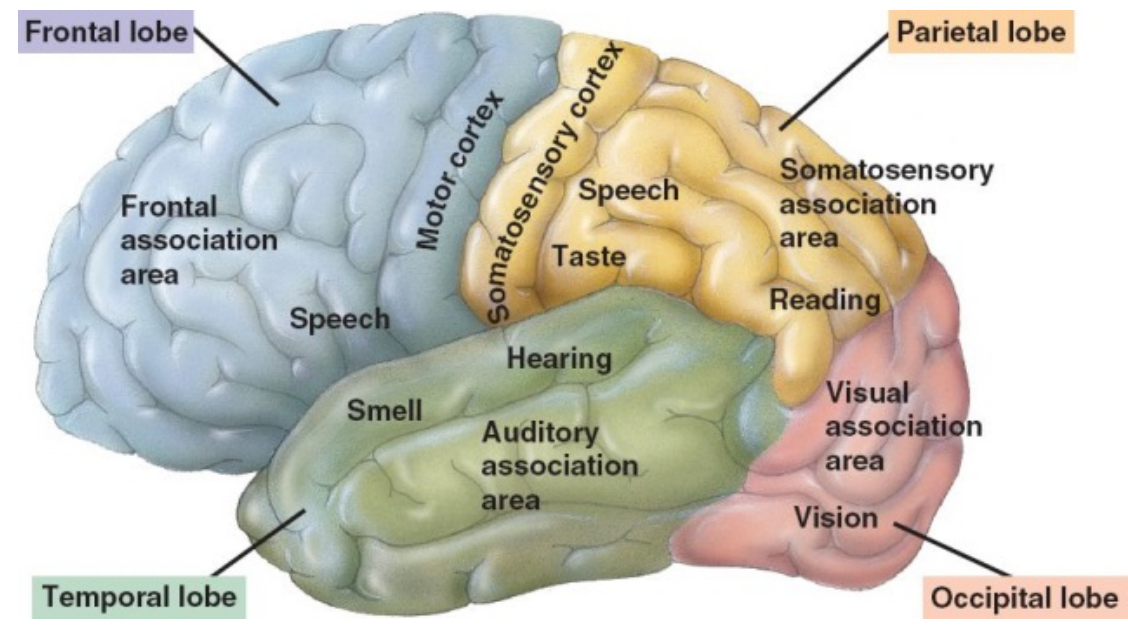

Figure 2-2. Functional regions of the human cerebral cortex. The somatosensory and motor areas are located between the frontal and parietal lobes of the cerebral cortex.

detected. Therefore, MI-based BCIs can be used by imagining movements of the left hand, right hand, feet, and tongue (Schlögl et al. 2005).

ERD/ERS patterns are generated from motor and somatosensory areas, which are directly connected to the normal neuromuscular output pathways. For that reason, motor imagery is a suitable mental strategy for BCIs. After several training sessions with a MI-based BCI, individuals can learn and develop their own motor imagery strategy. In cursor movement tasks, users learn which types of imagined movements are best for reliably moving the cursor up or down. Some subjects learn to move a cursor in two (Fabiani et al. 2004) or even three (Schlögl et al. 2005, Fabiani et al. 2004, McFarland et al. 2008) dimensions with further training. Users can utilize the feedback to optimize their mental tasks and improve BCI performance. The feedback indicates whether the user performed the mental task well or failed to reach the desired goal through the BCI. The feedback is typically visual but it can also be tactile or auditory (Neuper and Pfurtscheller 2010). Figure 2-3 shows the amplitude of the brain rhythms when a user tries to move a cursor to a target located at the top or at the bottom of the screen. The control is clearly focused in the $\mu$ frequency band.

Likewise SCPs-based BCIs, MI-based BCIs do not depend on external stimuli. Nevertheless, motor imagery is a skill that has to be learned. While performance and training time vary across subjects, most subjects can reach proper control in a 2-choice task with 1-4 h of training (Blankertz et al. 2010, Pfurtscheller et al. 2010, Sellers et al. 2010). Although MI-based BCIs allows best performance and 


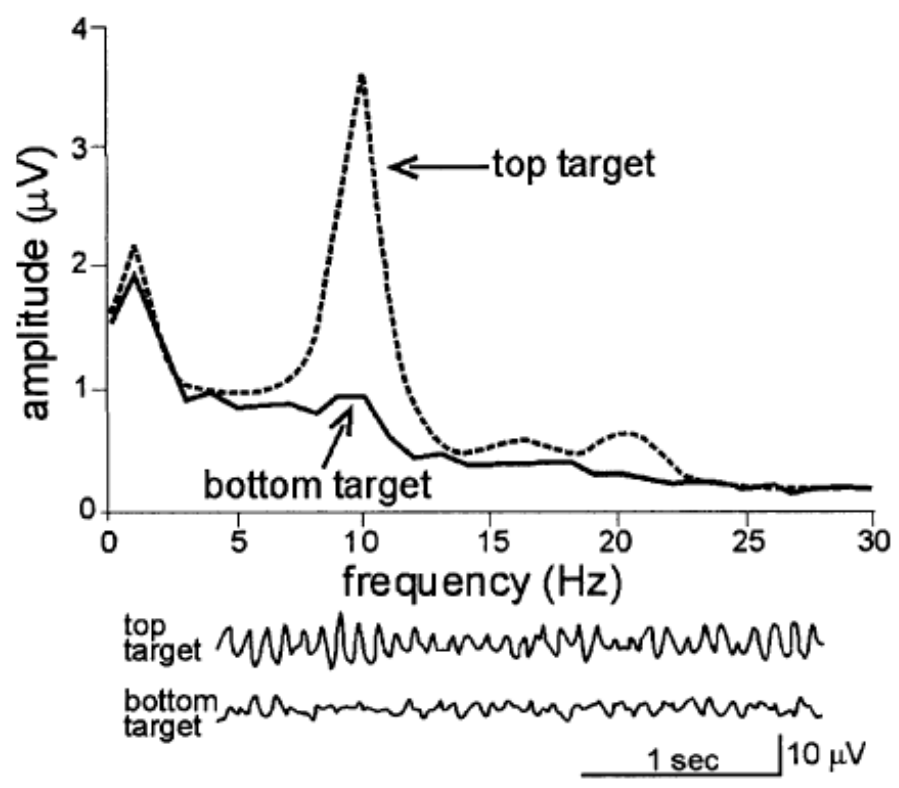

Figure 2-3. The user can control the amplitude of the $\mu$ rhythm (or the $\beta$ rhythm) in MIbased BCIs in order to move a cursor to a target located at the top or at the bottom of the screen. Frequency spectra show that control is clearly focused in the $\mu$ rhythm frequency band. (Wolpaw et al. 2002).

higher information rates than SCPs-based BCIs (Wolpaw et al. 2002), longer training is often necessary to achieve sufficient control. Holz et al. 2013 developed a MI-based game for BCI potential end-users. Only two out of four end-users were able to suitably play the game and the accuracies they reached were moderate. Extensive training required for MI-based BCIs makes the workload strong and thus they could not be the most proper systems for impaired people.

\subsubsection{Steady-State Visual Evoked Potentials (SSVEPs)}

Exogenous BCIs require external stimuli provided by the BCI system. Most BCIs are based on visual stimuli. Nevertheless, the stimuli can also be auditory (Klobassa et al. 2009) or somatosensory (Müller-Putz et al. 2006). Therefore, the stimuli can be different tones, different tactile stimulations or flashing lights at different frequencies. Typically, each stimulus is related to a specific command that controls the BCI application. The items could represent a wide variety of desired commands, such as letters, words, commands to move a wheelchair or a prosthesis, or to control a smart home. The users have to focus their attention to the corresponding stimulus in order to select a command. 
Visual stimuli-based BCIs can be implemented using two different patterns: steady-state visual evoked potentials (SSVEPs) and P300 evoked potentials. A SSVEPbased BCI requires a number of visual stimuli. Each stimulus is associated with a specific command related to an action that the BCI can perform. These stimuli do not flash consecutively, but flicker continuously at different frequencies in the range of about 6-30 Hz. Paying attention to one of the flickering stimuli, a SSVEP is evoked in the visual cerebral cortex. Moreover, this potential presents the same frequency as the target flashes. Thus, if the desired command related stimulus flickers at $12 \mathrm{~Hz}$, the elicited SSVEP will also flicker at $12 \mathrm{~Hz}$. Therefore, a SSVEP-based BCI can determine the user's desired command by looking for SSVEPs in the brain activity over the visual cortex at specific frequencies.

\subsubsection{P300 evoked potentials}

Likewise SSVEP-based BCIs, a BCI based on P300 commonly uses visual stimuli. A P300-based BCI consists in stimuli that flash in random sequence. The stimuli are usually letters but they can be symbols or images representing other goals, such as movement of a cursor, of a prosthesis, or a mobile robot (Bell et al. 2008, Citi et al. 2008). Selective attention to a specific flashing letter/symbol/image evokes a brain pattern called P300, which appears around the central and parietal brain cortex, close to electrode location $\mathrm{Pz}$, about $300 \mathrm{~ms}$ after the stimulus presentation. Figure 2-4 shows the averaged responses for attended and unattended stimuli. The BCI can detect which specific stimuli elicited this P300 and subsequently determine the desired letter/symbol/image.

BCI approaches using P300 are quite reliable across different users and sessions and can enable reasonably fast communication. Additionally, these systems do not require significant training, since users can generate evoked potentials without any previous training. Standard visual stimuli necessary to elicit this exogenous activity may depend to some degree on control of eye gaze (Corralejo et al. 2014). Although P300based BCIs without gaze shifting are also possible (Allison et al. 2008), locked-in patients or subjects with poor control of eye movements might achieve better communication using tactile feedback (Wolpaw et al. 2000). 


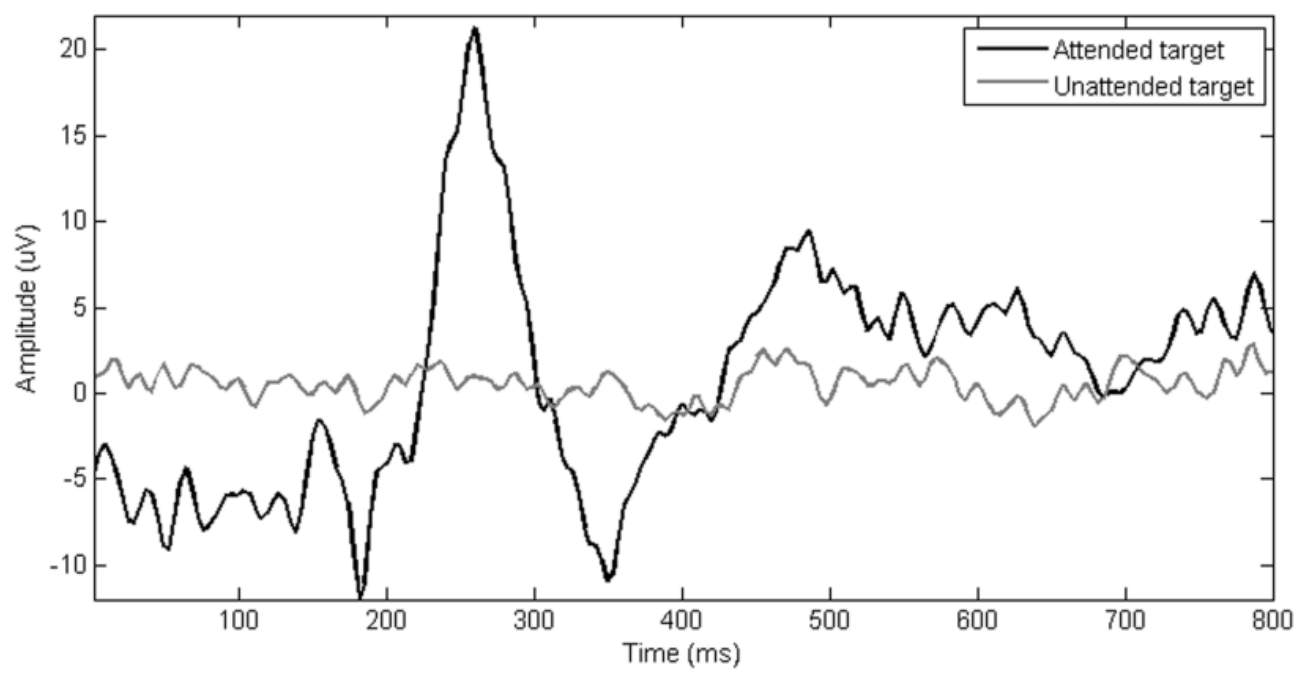

Figure 2-4. Time course at electrode location $\mathrm{Pz}$ of the voltage for attended (black line) and unattended (grey line) stimuli. The attended stimuli evoke a P300 response waveform.

This Doctoral Thesis proposes the use of P300-based BCIs in order to get practical assistive tools at home for severely impaired individuals. Most healthy subjects can reach high accuracy performance with P300-based BCIs after few minutes of training versus MI-based BCIs, which need longer training periods (Guger et al. 2009). Therefore, P300-based BCIs could be a proper type of BCI for severely disabled populations since they do not require extended training and they enable to select quickly the desired option among a large number of choices only by focusing on it.

\subsection{Basis and applications of P300-based BCIs}

P300 response is an event-related potential (ERP) elicited in the EEG brain activity over the parietal cortex. It is a typical, or naive, response to a desired choice (Donchin and Smith 1970). Infrequent or particularly significant auditory, visual or somatosensory stimuli, when interspersed with frequent or routine stimuli, typically elicit in the EEG a positive peak at about 300 ms (see Figure 2-4). Specifically, when subjects perform a task that requires them to determine to which of two possible categories each item in a series belongs, and one of the categories occurs rarely, these infrequent items evoke an ERP with an enhanced component with a latency of about 300 ms (Farwell and Donchin 1988). This elicited component is the so-called P300 
evoked response. An experiment based on this response is known as oddball paradigm (Farwell and Donchin 1988, Hillyard and Kutas 1983, Pritchard 1981).

It has been amply documented that the amplitude of the P300 varies directly with the relevance of the eliciting events and inversely with the probability of the stimuli (Farwell and Donchin 1988). Consequently, the induction of P300 responses depends on the user's ability to discriminate the different events and to assign them to the appropriate categories. It is not necessary that the user report the occurrence of the desired event (e.g. by pressing a button). Typically, the user is required only to maintain a running mental count of the number of occurrences of the desired event (Farwell and Donchin 1988). In other words, the appearance of the P300 response depends on the user's ability to recognize the rare events without recourse to verbal or motor means of communication (Farwell and Donchin 1988).

The discrimination between the event on which the subject is focusing and the other events provides the user's intention. By identifying which of the events generates a P300 response, an application can communicate or perform the desired command. Therefore, P300-based BCIs enable to select among several items displayed in the screen using this 'oddball' response (Donchin et al. 2000, Farwell and Donchin 1988). The typical experiment consists in a stimuli matrix that shows an array with the different items/choices in the screen. Usually, each stimulus consists in the flashing of a single row or column of this matrix. When the user's desired item flashes, an evoked potential response appears in the subject’s EEG activity (Corralejo et al. 2014).

Research in P300-based BCIs includes different approaches. Firstly, many studies are focused on improving P300-based BCIs performance by improving the feature extraction and classification algorithms (Aloise et al. 2011a, Khan et al. 2012, Krusienski et al. 2006, Rakotomamonjy and Guigue 2008, Rivet et al. 2009, Serby et al. 2005, Xu et al. 2004). Secondly, other studies analyse the effects of different visual stimuli presentation paradigms (Jin et al. 2011, Jin et al. 2012, Mak et al. 2012, Salvaris and Sepulveda 2009, Sellers et al. 2006). Thirdly, other types of stimuli presentation, such as auditory stimuli (Klobassa et al. 2009) or tactile stimuli (Brouwer and van Erp 2010) were studied. Finally, other researchers worked on developing novel BCI applications (Citi et al. 2008, Corralejo et al. 2014, Fernández et al. 2013, Finke et al. 2009, Iturrate et al. 2009, Käthner et al. 2014, Mugler et al. 2010, Yu et al. 2012). 
The different applications of P300-based BCIs are presented in this subsection. Most of these studies were assessed by populations of healthy users. Nevertheless, the few studies that verify the usefulness of P300-based BCIs for potential end-users populations, i.e. severely disabled people, will be thoroughly described in the next subsection.

\subsubsection{Speller}

The first and most typical P300-based BCI application is the so-called P300 Speller. It allows users to select single letters and characters in order to create words and thus messages. Donchin et al. (2000) showed the user a matrix consisted of $6 \times 6$ characters (the alphabet letters from A to Z, the numbers from 1 to 9 and the space character). The rows and columns in the matrix flashed randomly and successively at a rate of eight flashes per second (one flash every $125 \mathrm{~ms}$ ). The user had to select a character and focus the attention on it. Then, the subject had to count how many times the desired item flashed, i.e.: how many times the row or the column containing the desired choice flashed. As the user focuses attention on that item, the row and column containing it become relevant events. There are 12 possible events (6 rows and 6 columns) but only 2 of them are relevant. These events are thus rare or infrequent. Therefore, any flash that contains the character on which the subject is focusing should evoke a P300, whereas the others should not. After averaging several responses, the computer can determine the row and column that evoked the P300 response (as the ones with the highest P300 amplitude) and thus the desired character (as the intersection of the row and the column that elicited the largest P300). Later, Schalk et al. (2004) implemented a P3Speller in the BCI2000 general-purpose system, as shown in Figure 25. This implementation includes a text bar at the top in order to provide the user visual feedback, i.e.: the selected letters are shown consecutively.

\subsubsection{Internet browsing}

Since Donchin et al. (2000) proposed the P300 Speller, several new applications arose from it. Whereas one approach modified the speller in order to publish the generated messages in Twitter (Fazel-Rezai et al. 2012), other exploited the BCI2000 P3Speller paradigm in order to create an Internet browser controlled by P300 


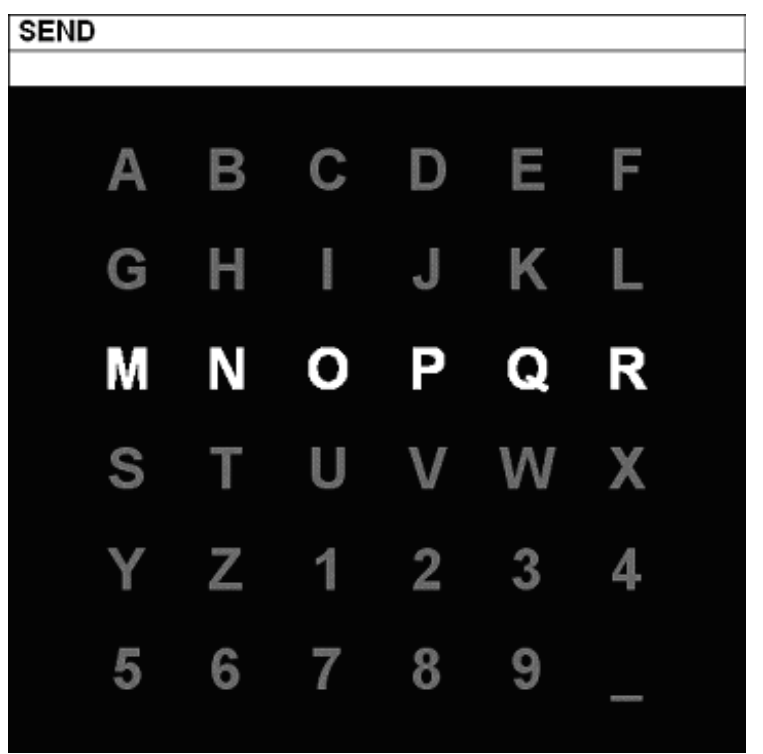

Figure 2-5. P3 Speller application developed by the BCI2000 team. In this frame the third row is flashed. (Schalk and Mellinger 2010).

evoked potentials. The browser proposed by Mugler et al. (2010) allows navigation (forward, back, reload and home), data form entry, address bar entry and scroll up and scroll down. These functions are all presented as icons within a stimuli matrix consisted of 8 x 8 items, as illustrated in Figure 2-6. By using an alphabetic code for link tags, each link on the Web page can be executed by entering either a one-or two-letter command. For instance, the two-letter link tag BA can be executed by selecting first the command with an asterisk ' $\mathrm{B}^{*}$ ' and then the next letter ' $\mathrm{A}$ '. Consequently, for sites with more than 26 links on a page and up to 338 links, any link can be selected by executing two commands. That is much less number of commands than if the link selection method were binary. After each selection, visual feedback of the selected command is displayed to the users.

Control of computers has been also implemented by means of P300-based BCI mice. Citi et al. (2008) developed a P300-based BCI for the 2D (2 dimensions) control of a cursor on a computer screen. Four grey, small, unobtrusive and peripheral rectangles are continually superimposed on the screen, as shown in Figure 2-7. Each rectangle is associated with a possible direction of movement for the mouse. Two additional rectangles, one on the bottom left corner of the screen and the other on the bottom right corner, can also be present. When they are enabled, they perform the function of the left and right buttons of an ordinary mouse. When this function is not necessary, both rectangles are not shown on the screen to avoid distracting the user. The 


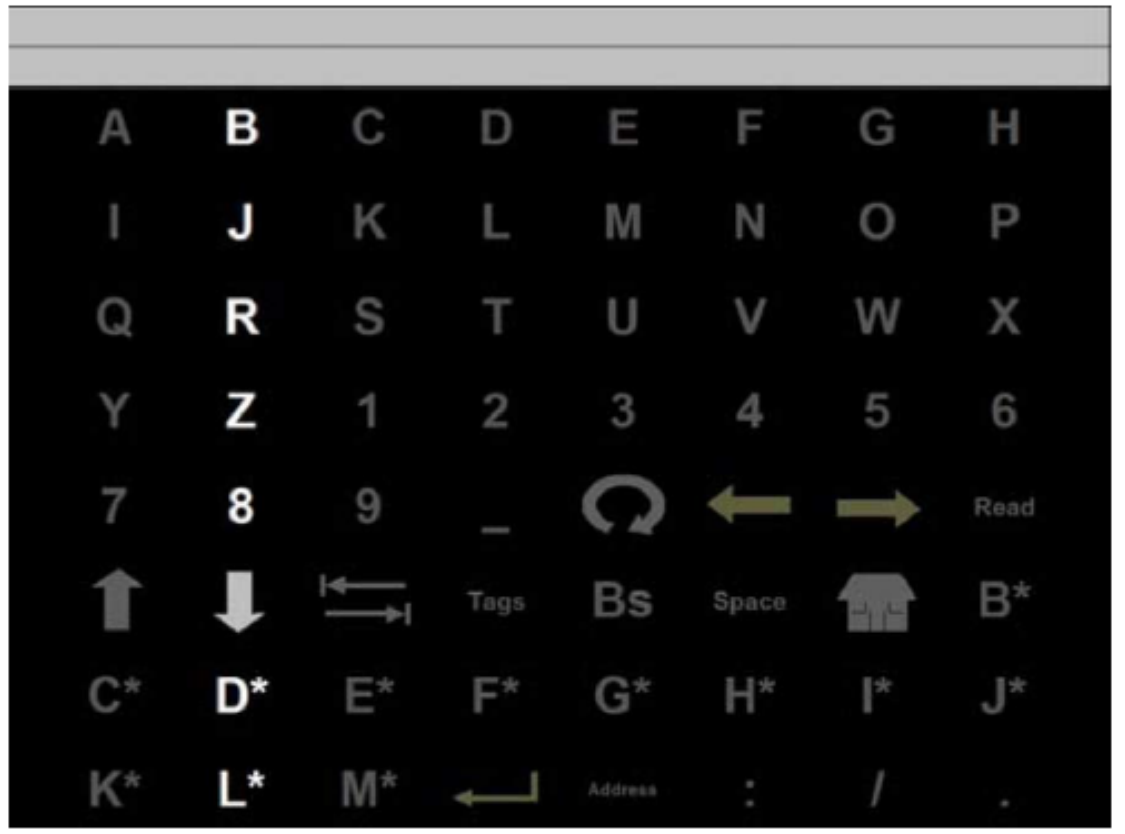

Figure 2-6. Stimuli matrix for P300 Browser. In this frame, the second column is flashed. (Mugler et al. 2010).

rectangles are flashed randomly (changing its colour from grey to bright red) while users focus their attention to the desired rectangle and count how many times it flashes. The generated EEG components corresponding to each stimulus are analysed in order determine the user's intention and move the cursor. Feedback is provided by means of the trajectory of the mouse pointer produced by own users. Other BCI mouse approach was developed by Yu et al. (2012). They proposed an advanced 2D BCI mouse. They filter the targets links of interest from the current Web page before the user move the mouse toward the desired link using his EEG brain activity. Horizontal movement is controlled by motor imagery whereas the vertical movement is controlled by P300 responses. The proposed browser allows common navigation functions, including history rolling forward and backward, hyperlink selection, page scrolling, text input, etc.

\subsubsection{Wheelchairs}

P300 paradigm has been also applied to control the movement of wheelchairs. Iturrate et al. (2009) proposed a P300-based BCI for manage an advanced wheelchair. The user faces a screen displaying a real-time virtual reconstruction of the actual scenario, constructed by means of a laser scanner (see Figure 2-8). The user has 


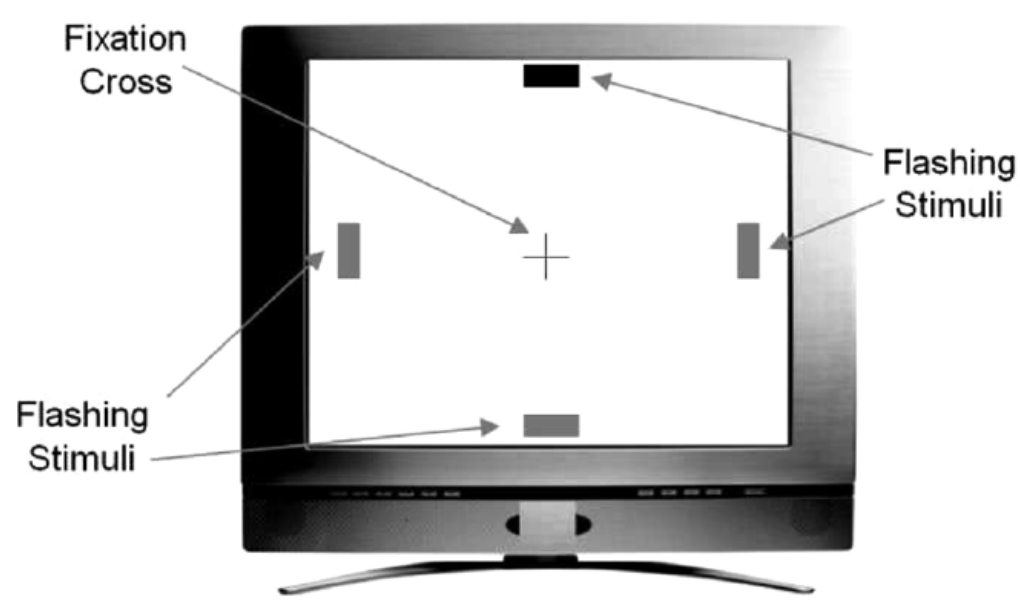

Figure 2-7. P300-based BCI mouse developed by Citi et al. (2008). The four rectangles at the sides of the screen are associated with the possible movement directions. In this frame, the stimulus of the up direction is presented.
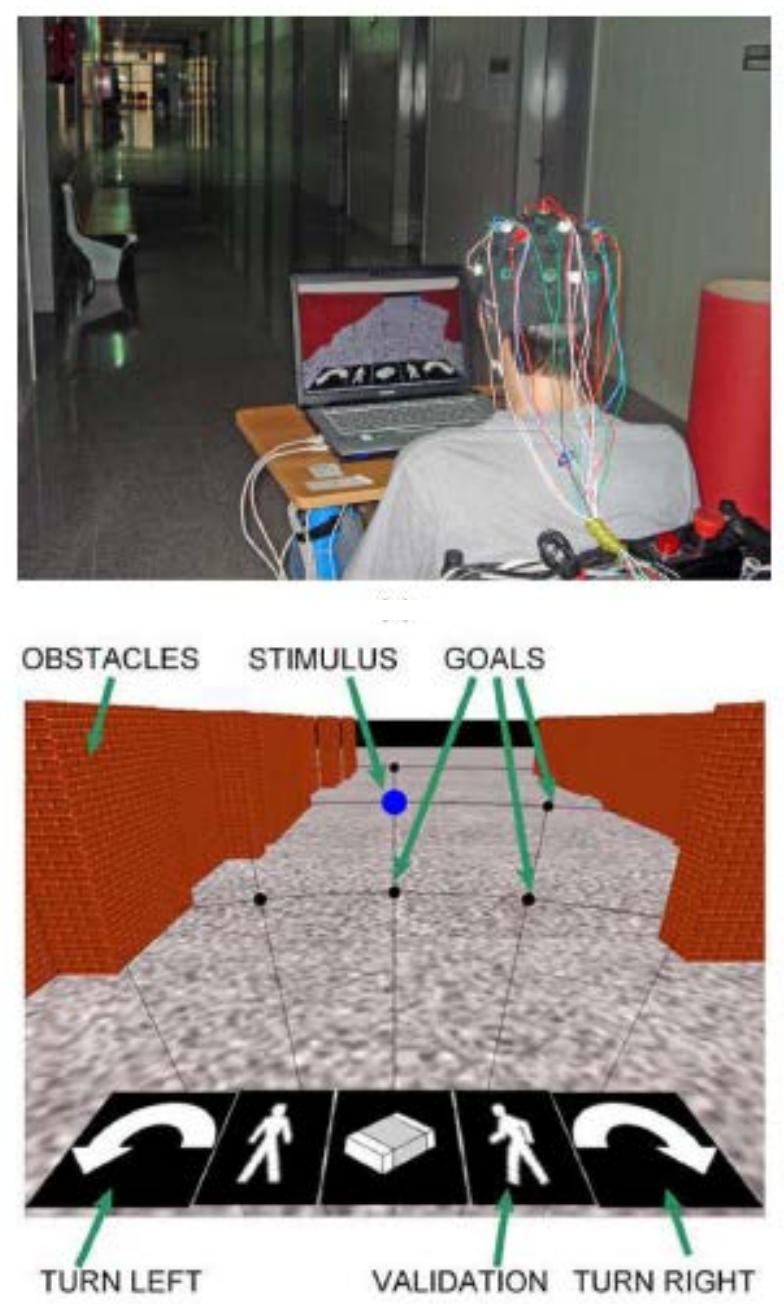

Figure 2-8. (At the top) Picture of a participant navigating with the BCI-controlled wheelchair implemented by Iturrate et al. (2009). (At the bottom) Screenshot of the screen showed to the user for selecting the desired commands. 
to focus on the location of the space to reach or the desired control command. A visual stimulation process evokes the P300 responses and thus the desired target can be identified. Then, the selected location is given to the autonomous navigation system that drives the wheelchair to the desired position avoiding collisions with the detected obstacles. This P300-based BCI application allows user to give a complex command and relax, since the wheelchair navigation is safe and automated, and avoid the exhausting mental processes of selecting a series of commands for each single movement.

\subsubsection{Entertainment}

Finally, entertainment approaches have also been developed by means of P300-based BCIs. Finke et al. (2009) developed the MindGame, a BCI game based on the original P300 Speller paradigm. The MindGame is played on a checkerboard-styled board with 28 x 18 cells and 12 randomly positioned trees. The user is represented by an avatar that can move from field to field. Fields with trees are potential target stimuli in terms of the oddball paradigm. The player has to move the avatar from tree to tree, until each tree has been visited. The movement is performed by exploiting the trees as potential target stimuli, analogously to the characters in the stimuli matrix of the original P300 Speller paradigm. Instead of the usual row/column (RC) stimuli pattern, one trial consists of the consecutive highlighting of all 12 target fields. Target and background stimuli are coded by different colours: red for the target and yellow for the non-targets. The player focuses his attention on the target, and the red flash will evoke a P300 response while the yellow flashes will not. Then, the game will identify the user's desired movement and the avatar will be consequently moved. The graphical setup of this BCI game is presented in Figure 2-9.

In summary, Table 2-1 gathers the main features of each application described in this subsection as well as the size of the population and the results of each study. 


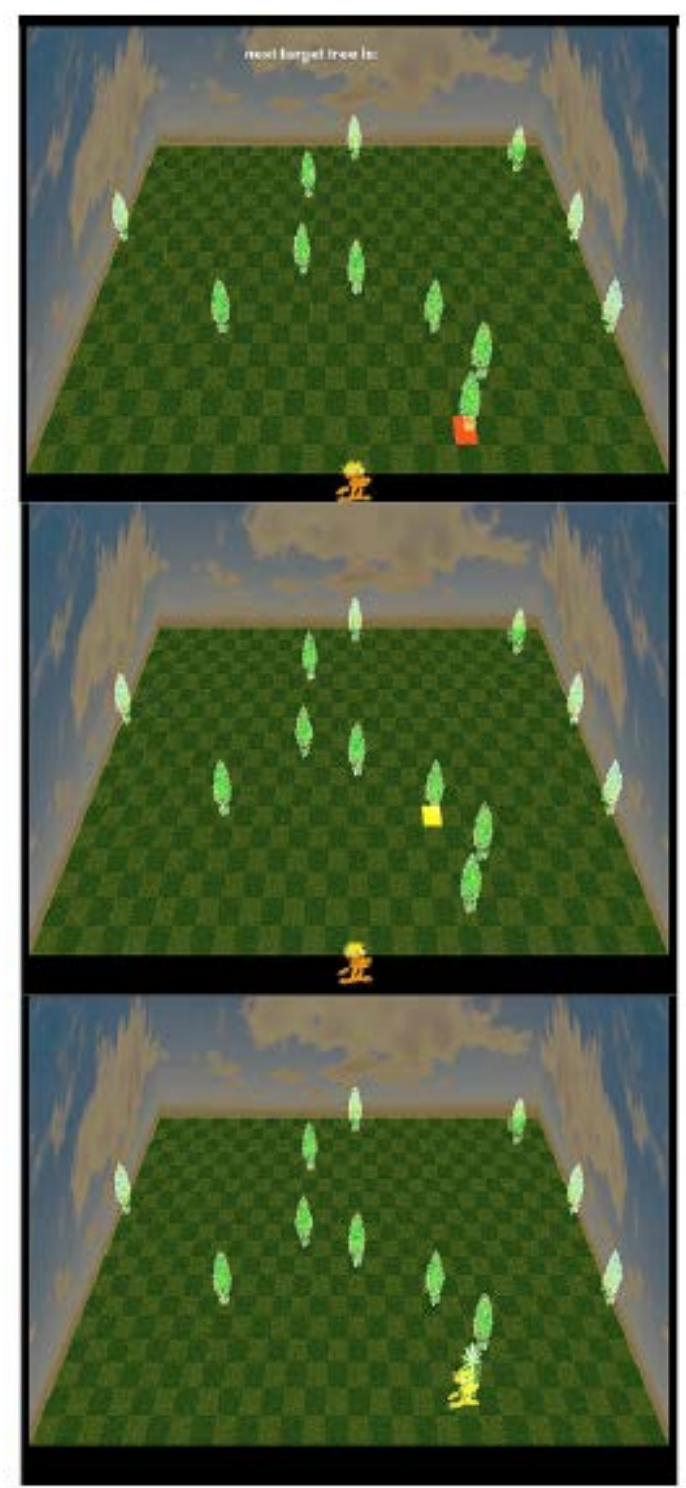

Figure 2-9. Screenshots from the MindGame (Finke et al. 2009). The player controls the avatar by selecting the target of the next move. 


\begin{tabular}{|c|c|c|c|}
\hline Authors & Subjects & Application & $A C C(\%)$ \\
\hline Donchin et al. 2000 & 10 & $\begin{array}{l}\text { Speller } \\
\text { RC stimuli pattern } \\
\text { Matrix of characters } \\
6 x 6\end{array}$ & $56-90$ \\
\hline Fazel-Rezai et al. 2012 & 1 & $\begin{array}{l}\text { Twitter messaging } \\
\text { RC stimuli pattern } \\
\text { Matrix of characters/words } \\
\text { 9x6 }\end{array}$ & 87 \\
\hline Mugler et al. 2010 & 10 & $\begin{array}{l}\text { Internet browser } \\
\text { RC stimuli pattern } \\
\text { Matrix of characters/words } \\
8 \text { x8 }\end{array}$ & 90 \\
\hline Citi et al. 2008 & 5 & $\begin{array}{l}\text { Computer mouse } \\
\text { One to one stimuli pattern } \\
4 \text { choices }\end{array}$ & $\begin{array}{c}\text { Not } \\
\text { provided }\end{array}$ \\
\hline Yu et al. 2012 & 7 & $\begin{array}{l}\text { Computer mouse: P300 for vertical } \\
\text { and MI for horizontal movement } \\
8 \text { choices }\end{array}$ & 93 \\
\hline Iturrate et al. 2009 & 5 & $\begin{array}{l}\text { Wheelchair } \\
\text { RC stimuli pattern } \\
\text { Matrix of images } \\
4 \times 5\end{array}$ & 92 \\
\hline Finke et al. 2009 & 11 & $\begin{array}{l}\text { Entertainment } \\
\text { One to one stimuli pattern } \\
\text { Matrix of images } \\
13 \text { choices }\end{array}$ & 66 \\
\hline
\end{tabular}

Table 2-1. Summary of different studies for novel P300-based applications. Number of healthy subjects, main details of each work and the mean online accuracy (ACC) are shown. 


\subsection{P300-based BCIs involving disabled populations}

A BCI monitors brain activity and translates the user's intent into control commands. Therefore, a BCI provides a new augmentative communication technology to those individuals who have severe movement deficits or are paralyzed. Other augmentative communication technologies require some form of muscle control and thus could not be useful for those with the most severe motor impairments. Many different disorders can disrupt the channels through which the brain normally communicates with and controls its external environment (Wolpaw et al. 2002). ALS, brainstem stroke, brain or spinal cord injury, cerebral palsy, muscular dystrophies, multiple sclerosis (MS) and other degenerative diseases impair the neural pathways that control muscles or impair the muscles themselves. BCIs allow these individuals to express their wishes, feelings and needs to the outside world and to increase their personal autonomy. Although severely impaired people are the real end-users of BCIs, few studies verify the usefulness of BCIs with disabled populations. This subsection is consequently focused on the studies that involve P300-based BCIs and impaired users.

Sellers and Donchin (2006) applied P300 potentials to develop a 4-choice communicator. The study included 3 ALS patients and 3 healthy subjects. The available choices were: Yes, No, Pass and End. Each individual performed 10 experimental sessions during a period of approximately 6 weeks. During each run the participant's task was to attend to one stimulus and disregard the other three. Stimuli were presented in auditory mode, in visual mode or in both modes. It was found that individuals with advanced ALS could use a P300-based BCI using auditory and/or visual stimuli with mean accuracy (ACC) of 65\% and information transfer rate (ITR) of $0.7 \mathrm{bit} / \mathrm{min}$.

There are several works that investigate the P300 Speller performance in disabled populations. Firstly, Nijboer et al. (2008) studied the efficacy of P300-based BCIs for 6 individuals with advanced ALS. Participants focused on one character of a 6 x 6 or a $7 \times 7$ matrix while the rows and columns flashed randomly. Four out of the participants completed the experiment. They were able to use a P300-based BCI for cued and spontaneous text production with mean ACC of $72 \%$ and ITR of $8.4 \mathrm{bit} / \mathrm{min}$. Moreover, Nijboer et al. (2008) performed an exhaustive study and showed that the amplitude and latency of the P300 potential of these patients remained steady over 40 weeks. Secondly, Ortner et al. (2011) analysed the P300 Speller performance of a young healthy population and a group of 15 people with severe motor disabilities. 
Subjects attended to one character of a $10 \times 5$ or a $6 \times 6$ matrix while the rows and columns flashed randomly. The patient group reached mean ACC of $71 \%$ in comparison to $91 \%$ achieved for the group of control subjects. Furthermore, the study determines that properly adjust the parameters of the environment and the paradigm for each patient could be essential for regular use of BCIs. Thirdly, Mak et al. (2012) identified EEG features that correlate with P300-based BCI performance in a population of twenty patients with advanced ALS. Participants focused on one character of a 6 x 6 matrix while groups of items in the speller matrix flashed randomly using the checkerboard (CB) stimuli pattern (Townsend et al. 2010). In the CB pattern the stimuli matrix is virtually superimposed on a checkerboard, which the participants never actually see. The items are segregated into two virtual smaller matrices according their corresponding colour in the checkerboard (white or black). During one sequence, the virtual rows in the white matrix flash in order from top to bottom followed by the virtual rows in the black matrix. Then, the virtual columns in the white matrix flash in order from left to right followed by the virtual columns in the black matrix. The result is that the users see random groups of items flashing, instead of rows and columns, as can be seen in Figure 2-10. The virtual checkerboard layout reduces adjacency-distraction errors because adjacent items are never included in the same flash group (Townsend et al. 2010). Eleven out of the twenty participants achieved ACC of 100\% during at least one copy-spelling run. Nevertheless, the mean ACC over sessions and patients was

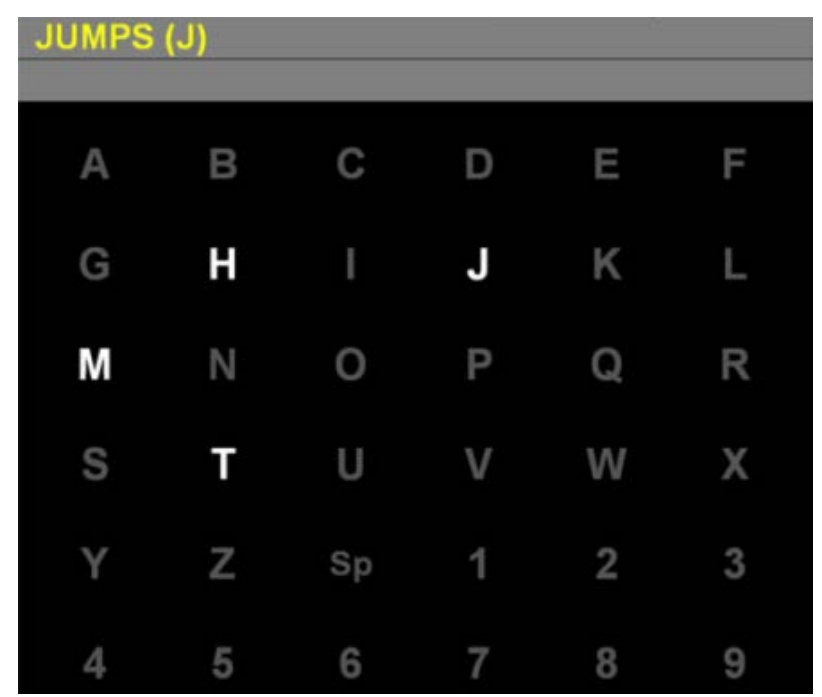

Figure 2-10. Stimuli matrix used in the study of Mak et al. (2012). Groups of items in the speller matrix flashed successively and randomly using the CB paradigm. (Modified from Mak et al. 2012). 
58\%. Finally, Spüler et al. (2012) investigated whether error-related potentials could be useful to increase the ITR of the P300 Speller in 17 healthy subjects and 6 ALS patients. Participants focused on one character of a $6 \times 6$ matrix while the rows and columns flashed randomly, as shown in Figure 2-11. On recognition of error-related potentials, the interface notified the user that the incorrect letter was automatically deleted. The disabled population achieved a mean ACC of 74\% and ITR of $2.1 \mathrm{bit} / \mathrm{min}$ applying this methodology. A summary of these P300-based spellers involving real end-users is summarized in Table 2-2.

In regard to studies involving P300-based BCIs and end-users, several applications for home automation control were developed. Hoffman et al. (2008) evaluated a P300-based BCI with five disabled subjects due to different pathologies and four healthy individuals. The proposed tool simulated an environment control paradigm. Participants focused on one image of a $3 \times 2$ matrix while the single items flashed randomly, as can be seen in Figure 2-12. Only offline performance results are provided.

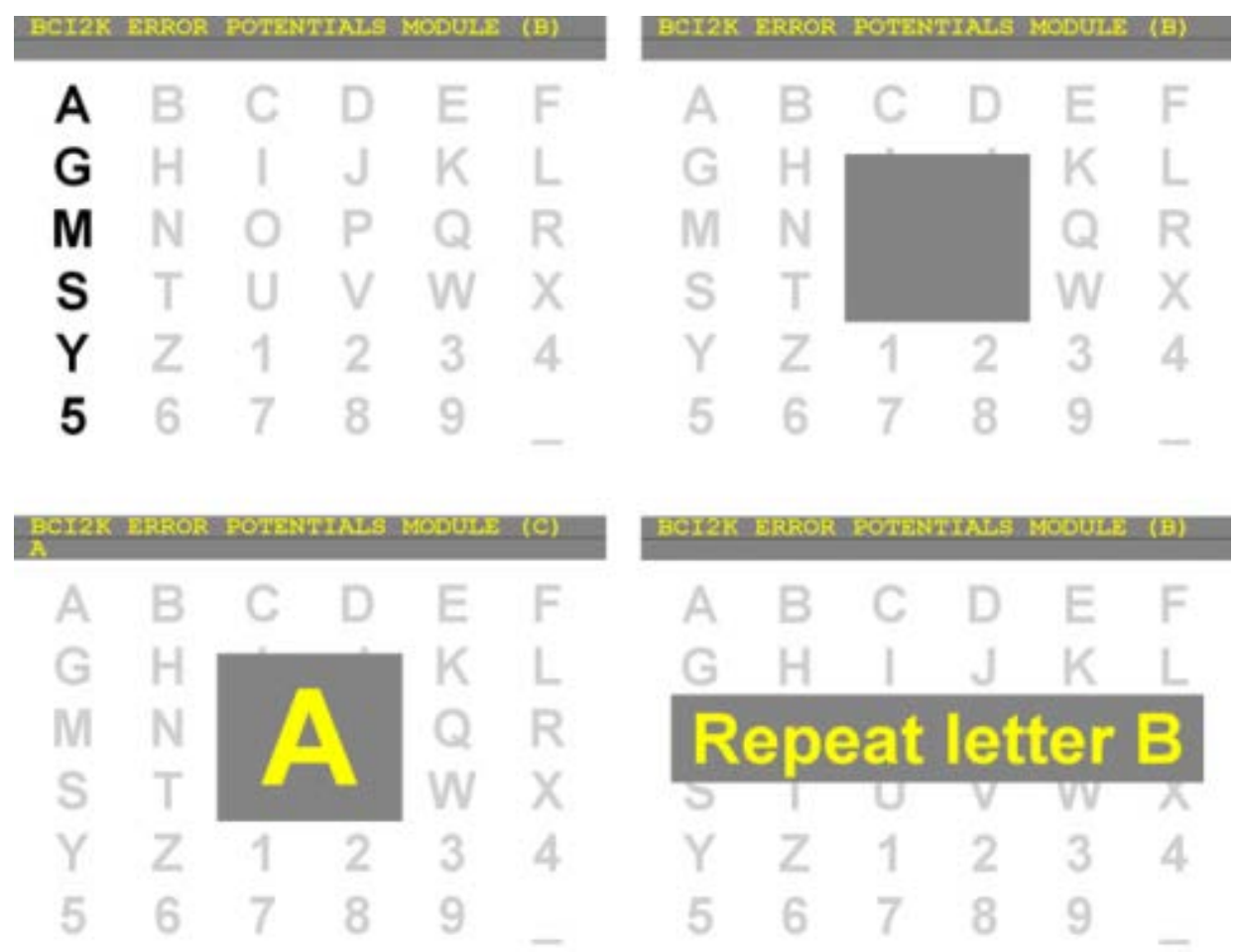

Figure 2-11. Paradigm of the study of Spüler et al. (2012). Top left: rows and columns flash randomly as in the typical speller paradigm. Top right: after classification, a grey square is shown for $1 \mathrm{~s}$. Bottom left: the selected letter is shown to the user for another second. Bottom right: if error-related potentials are detected, the letter is removed and a notification appears. (Spüler et al. 2012). 


\begin{tabular}{|c|c|c|c|c|}
\hline \multicolumn{5}{|c|}{ Speller } \\
\hline Authors & Subjects & Application & $\begin{array}{c}\text { Mean } \\
\text { ACC (\%) }\end{array}$ & $\begin{array}{c}\text { ITR } \\
\text { (bit/min) }\end{array}$ \\
\hline Sellers and & & One to one stimuli pattern & & \\
\hline Donchin & 3 & 4 choices (words) & 64.8 & 0.7 \\
\hline (2006) & & Visual and/or auditive stimuli & & \\
\hline $\begin{array}{l}\text { Nijboer et al. } \\
\text { (2008) }\end{array}$ & $4^{*}$ & $\begin{array}{l}\text { RC stimuli pattern } \\
\text { Matrix of characters } \\
6 x 6 \text { or } 7 \times 7\end{array}$ & 71.9 & 8.4 \\
\hline $\begin{array}{l}\text { Ortner et al. } \\
\text { (2011) }\end{array}$ & 15 & $\begin{array}{l}\text { RC stimuli pattern } \\
\text { Matrix of characters } \\
10 x 5 \text { or } 6 \times 6\end{array}$ & 70.7 & - \\
\hline $\begin{array}{l}\text { Spüler et al. } \\
\text { (2012) }\end{array}$ & 6 & $\begin{array}{l}\text { RC stimuli pattern } \\
\text { Matrix of characters } \\
6 \times 6 \\
\text { Includes error potentials detection }\end{array}$ & 74.0 & 2.1 \\
\hline $\begin{array}{l}\text { Mak et al. } \\
\text { (2012) }\end{array}$ & 20 & $\begin{array}{l}\text { Checkerboard stimuli pattern } \\
\text { Matrix of characters } \\
6 x 6\end{array}$ & 58.0 & - \\
\hline
\end{tabular}

Table 2-2. Comparison of current studies involving P300-based spellers and disabled participants. Number of impaired subjects, main details of each study, mean online accuracy (ACC) and maximum information transfer rate (ITR) are provided.

${ }^{*}$ Four additional subjects were not included since they did not complete the study.

Four out of the five disabled participants achieved $100 \%$ offline ACC, as well as two out of the healthy subjects. However, in terms of ITR, healthy subjects reached higher maximum bitrates than impaired subjects. More recently, Aloise et al. (2011b) assessed an asynchronous P300-based BCI for virtual environmental control with seven potential end-users with limited autonomy due to ageing and/or motor disabilities. Participants attended to one image of a 4 x 4 matrix while the rows and columns flashed randomly. The proposed virtual environment is shown in Figure 2-13. Potential end-users reached $73 \%$ mean ACC and 1\% error rate in the asynchronous mode. The asynchronous modality performed significantly better as compared to the synchronous mode. Furthermore, the asynchronous system modality confirmed its reliability in avoiding misclassifications and false positives. A comparison of these applications for environment control involving real end-users is summarized in Table 2-3.

In summary, studies involving potential BCI end-users focused on the P300 Speller typical paradigm or developed virtual or simulated environment control tools. All these studies presented the user only one stimuli matrix with a limited number of choices. Additionally, these works showed there can be great differences between 

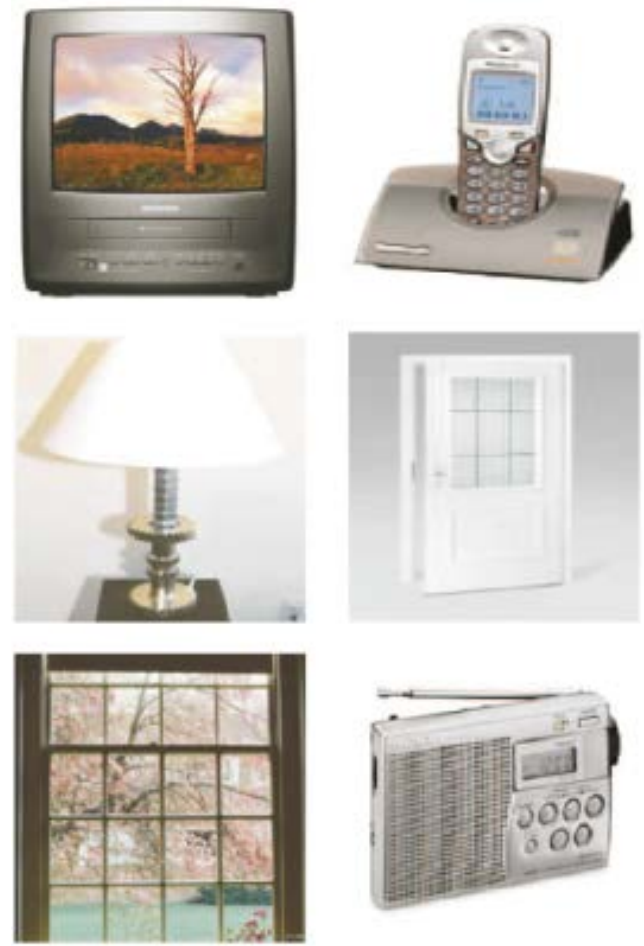

Figure 2-12. Stimuli matrix used by Hoffman et al. (2008) consisted of 3 x 2 images depicting devices present at home. Images were flashed, one at a time, by changing the overall brightness of images. (Hoffman et al. 2008).

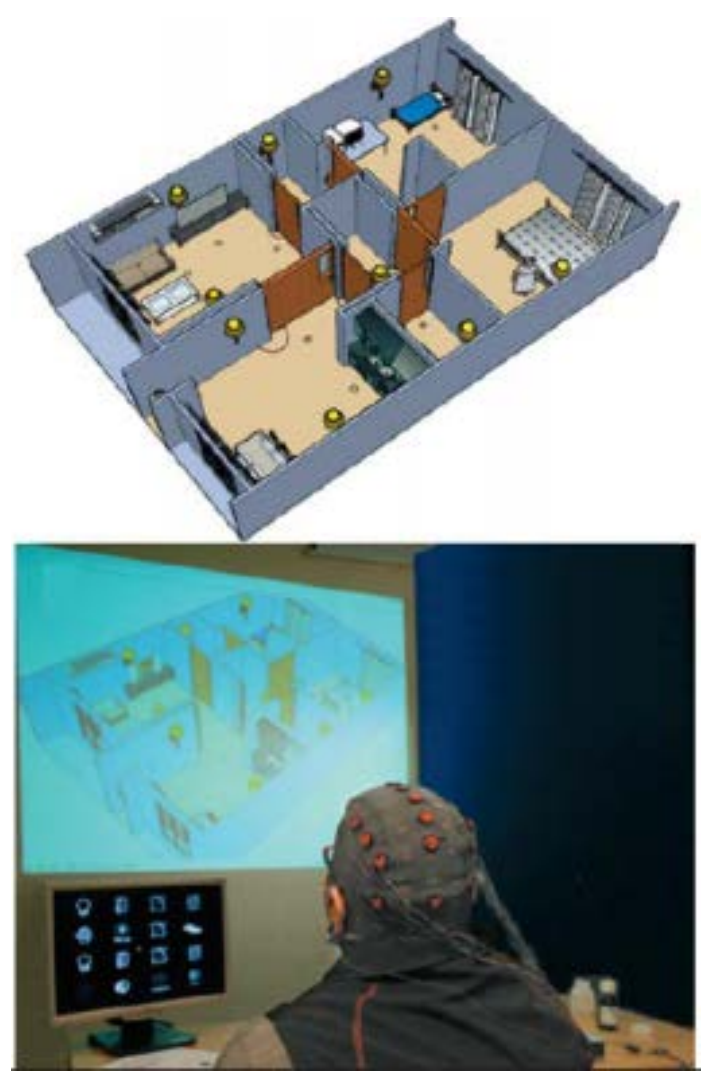

Figure 2-13. Top: Virtual environment used by Aloise et al. (2011b). Users can virtually operate lights, doors, curtains, windows, bed, TV, air condition, alarm and pause the system. Bottom: Picture of a user during the experimental session. The feedback was visually provided as changes in the virtual apartment. 


\begin{tabular}{|c|c|c|c|c|}
\hline \multicolumn{5}{|c|}{ Environment Control } \\
\hline Authors & Subjects & Application & $\begin{array}{c}\text { Mean } \\
A C C(\%)\end{array}$ & $\begin{array}{c}\text { ITR } \\
\text { (bit/min) }\end{array}$ \\
\hline $\begin{array}{l}\text { Hoffman et } \\
\text { al. (2008) }\end{array}$ & $4^{*}$ & $\begin{array}{l}\text { One to one stimuli pattern } \\
\text { Matrix of images } \\
3 x 2 \\
\text { Simulated environment control }\end{array}$ & $100.0^{* *}$ & $15.9^{* *}$ \\
\hline $\begin{array}{l}\text { Aloise et al. } \\
\text { (2011b) }\end{array}$ & 7 & $\begin{array}{l}\text { RC stimuli pattern } \\
\text { Matrix of images } \\
4 \times 4 \\
\text { Virtual environment control } \\
\text { Asynchornoous ooperation }\end{array}$ & 72.7 & - \\
\hline
\end{tabular}

Table 2-3. Comparison of different studies involving P300-based applications for environment control and disabled participants. Number of impaired subjects, main details of each study, mean online accuracy (ACC) and maximum information transfer rate (ITR) are shown.

* One additional subject was not included since classification accuracies above chance level could not be obtained.

${ }^{* *}$ Only offline classification results are provided. Four-fold cross-validation was used to estimate the accuracy.

performance of healthy subjects and patients. Consequently, larger end-users populations should be considered in future studies. Moreover, some studies suggest that some adaptations in the environment and paradigm could be necessary for improving the performance of each specific individual. 



\section{Materials and methods}

3.1. Population under study

3.2. EEG recording

3.3. EEG signal processing

3.3.1. Feature Extraction

3.3.2. Feature Selection

3.3.3. Feature Classification 



\subsection{Population under study}

The population available for this study was composed of 30 subjects with motor impairments. There was no distinction about the type of pathologies that caused the motor disabilities for recruitment of subjects. Therefore, some of them also presented cognitive impairments. All subjects were patients from the Spanish NRCD\&D or National Reference Centre on Disability and Dependence (Centro de Referencia Estatal de Discapacidad y Dependencia), located in San Andrés del Rabanedo (León, Spain). This Centre is supported by the Social Security Administration Body responsible for handling Social Services and which deals with older and/or dependent people (IMSERSO, Instituto de Mayores y Servicios Sociales). All participants in the study were BCI-naives (without any P300-based BCI previous experience). The study was approved by the local ethics committee. All subjects gave their informed consent for participation in the study.

Participants from the NRC-D\&D were recruited in two stages. Firstly, a group of 15 patients (7 males; 8 females; age: $49.40 \pm 9.79$ years; range: 35-67 years). Secondly, a new group of 15 subjects (11 males; 4 females; age: $47.00 \pm 11.38$ years; range: 26-65 years). The whole group of participants is thus composed of 18 males and 12 females. Their range age is 26-67 years and their mean \pm standard deviation age is $48.20 \pm 10.50$ years. The percentage of males was slightly higher in the population of patients (60\%). Demographic and clinical data for the population of this study are summarized in Table 3-1 and Table 3-2. Despite of his severe diagnosis, participant P1 did not show motor impairments at the time of the study.

\subsection{EEG recording}

EEG was recorded for each subject during the online BCI sessions with an approximate duration of one hour or one hour and a half. Eight channels (Fz, Cz, P3, Pz, P4, PO7, PO8 and Oz) were recorded according to the modified international 10-20 system (Jasper 1958, Jurcak et al. 2007). Figure 3-1 shows this EEG recording montage. The proposed electrode distribution covers the central, parietal and occipital cerebral cortex. Therefore, it allows picking up the P300 proper evoked potential, centred around $\mathrm{Cz}$, and additional evoked potentials, which are elicited by the visual stimuli over the 


\begin{tabular}{|c|c|c|c|c|c|c|}
\hline Participant & Sex & Age & Diagnosis & $\begin{array}{c}\text { Motor } \\
\text { Impairment } \\
\text { Degree }\end{array}$ & $\begin{array}{l}\text { Cognitive } \\
\text { Impairment } \\
\text { Degree }\end{array}$ & $\begin{array}{c}\text { Sustained } \\
\text { Attention } \\
\text { Ability }\end{array}$ \\
\hline P1 & $\mathrm{M}$ & 60 & Arnold-Chiari malformation & $\mathrm{A}$ & $\mathrm{A}$ & VG \\
\hline P2 & $\mathrm{F}$ & 43 & $\begin{array}{l}\text { Acquired brain injury, spastic } \\
\text { tetraparesis }\end{array}$ & S & $\mathrm{m}$ & G \\
\hline P3 & $\mathrm{F}$ & 36 & Spastic cerebral palsy & S & $\mathrm{m}$ & M \\
\hline P4 & $\mathrm{F}$ & 51 & $\begin{array}{l}\text { Extrapyramidal syndrome, } \\
\text { dystonia and parkinsonism }\end{array}$ & M & $\mathrm{m}$ & G \\
\hline P5 & $\mathrm{F}$ & 50 & Acquired brain injury, hemiparesis & M & M & G \\
\hline P6 & M & 49 & Spinal cord injury & $\mathrm{S}$ & A & VG \\
\hline P7 & M & 56 & $\begin{array}{l}\text { Neurofibromatosis and severe } \\
\text { kyphoscoliosis }\end{array}$ & S & $\mathrm{m}$ & G \\
\hline P8 & M & 67 & Spastic cerebral palsy & S & $\mathrm{m}$ & G \\
\hline P9 & M & 64 & Spastic cerebral palsy & S & $\mathrm{m}$ & G \\
\hline P10 & M & 40 & $\begin{array}{l}\text { Acquired brain injury, cerebral } \\
\text { infarction }\end{array}$ & M & M & M \\
\hline P11 & $\mathrm{F}$ & 57 & Multiple sclerosis & S & M & $\mathrm{P}$ \\
\hline $\mathrm{P} 12$ & $\mathrm{~F}$ & 35 & Spastic cerebral palsy & $\mathrm{m}$ & $\mathrm{m}$ & VG \\
\hline $\mathrm{P} 13$ & M & 46 & Spastic cerebral palsy & $\mathrm{S}$ & $\mathrm{m}$ & G \\
\hline P14 & $\mathrm{F}$ & 45 & Acquired brain injury & S & M & G \\
\hline $\mathrm{P} 15$ & $\mathrm{~F}$ & 42 & Spastic cerebral palsy & $\mathrm{S}$ & M & $\mathrm{M}$ \\
\hline \multicolumn{3}{|c|}{ Age (years) } & \multicolumn{4}{|c|}{$49.40 \pm 9.79$} \\
\hline \multicolumn{2}{|c|}{ Males (\%) } & & \multicolumn{4}{|c|}{46.67} \\
\hline
\end{tabular}

Table 3-1. Demographic and clinical data of the first group of participants in the study.

Sex: male (M), female (F).

Motor/Cognitive impairment degree: absent (A), mild (m), moderate (M), severe (S), profound (P).

Sustained attention ability: very good (VG), good (G), moderate (M), poor (P), very poor (VP).

visual cortex (Schalk 2007). EEG data was amplified with a g.USBamp biosignal amplifier (Guger Technologies OG, Graz, Austria). Recordings were performed by means of eight g.LADYbird active electrodes (Guger Technologies OG, Graz, Austria). They were referenced to the right earlobe and grounded to channel Fpz through a g.GAMMAearclip electrode and a g.LADYbirdGND electrode (Guger Technologies OG, Graz, Austria), respectively. This equipment is shown in Figure 3-2. Moreover, a full description of the equipment and its technical specifications can be found in the Guger Technologies website (Guger Technologies 2014).

EEG data was sampled at $256 \mathrm{~Hz}$. Then, a bandpass filter between 0.1 and $60 \mathrm{~Hz}$ was applied. Furthermore, in order to remove the power line interference, signals were notch filtered at $50 \mathrm{~Hz}$. Signals were also spatial filtered by means of the Common 


\begin{tabular}{|c|c|c|c|c|c|c|}
\hline Participant & Sex & Age & Diagnosis & $\begin{array}{c}\text { Motor } \\
\text { Impairment } \\
\text { Degree }\end{array}$ & $\begin{array}{l}\text { Cognitive } \\
\text { Impairment } \\
\text { Degree }\end{array}$ & $\begin{array}{c}\text { Sustained } \\
\text { Attention } \\
\text { Ability }\end{array}$ \\
\hline P16 & $\mathrm{M}$ & 48 & Spinal cord injury & $\mathrm{M}$ & $\mathrm{m}$ & VG \\
\hline P17 & M & 38 & Spinal cord injury & $\mathrm{m}$ & A & VG \\
\hline $\mathrm{P} 18$ & M & 60 & Ataxia & M & A & VG \\
\hline P19 & M & 26 & Encephalopathy & $\mathrm{S}$ & A & VG \\
\hline $\mathrm{P} 20$ & M & 42 & Polymalformative syndrome & M & A & G \\
\hline $\mathrm{P} 21$ & $\mathrm{~F}$ & 65 & Spinal cord injury & M & M & G \\
\hline P22 & $\mathrm{F}$ & 46 & Spinal cord injury & M & A & G \\
\hline $\mathrm{P} 23$ & M & 48 & Spinal cord injury & $\mathrm{m}$ & $\mathrm{m}$ & M \\
\hline P24 & $\mathrm{F}$ & 63 & Muscular dystrophy & $\mathrm{m}$ & M & $\mathrm{P}$ \\
\hline P25 & M & 39 & Muscular dystrophy & M & A & VG \\
\hline P26 & M & 46 & Polymalformative syndrome & $\mathrm{m}$ & $\mathrm{m}$ & $\mathrm{VP}$ \\
\hline P27 & M & 57 & Muscular dystrophy & M & A & VG \\
\hline P28 & $\mathrm{F}$ & 56 & Spinal cord injury & M & A & G \\
\hline P29 & M & 37 & Spinal cord injury & M & A & VG \\
\hline P30 & $\mathrm{M}$ & 34 & Spinal cord injury & M & A & G \\
\hline \multicolumn{3}{|c|}{ Age (years) } & \multicolumn{4}{|c|}{$47.00 \pm 11.38$} \\
\hline \multicolumn{3}{|c|}{ Males (\%) } & \multicolumn{4}{|c|}{73.33} \\
\hline
\end{tabular}

Table 3-2. Demographic and clinical data of the second group of participants in the study.

Sex: male (M), female (F).

Motor/Cognitive impairment degree: absent (A), mild (m), moderate (M), severe (S), profound (P).

Sustained attention ability: very good (VG), good (G), moderate (M), poor (P), very poor (VP).

Average Reference (CAR) method. Data collection and design of experiments were controlled by BCI2000, a general-purpose BCI software platform (Schalk et al. 2004, Schalk and Mellinger 2010).

\subsection{EEG signal processing}

As it was described in the previous chapters, infrequent stimuli typically evoke a positive response in the EEG over parietal cortex about $300 \mathrm{~ms}$ after stimulus presentation (Donchin and Smith 1970, Sutton et al. 1965, Walter et al. 1964). This response, which is called the P300 evoked potential, can be used for manage a BCI system (Farwell and Donchin 1988, Schalk et al. 2004, Sellers and Donchin 2006). The user is presented to a matrix consisted of different items: characters, pictures, etc. For 


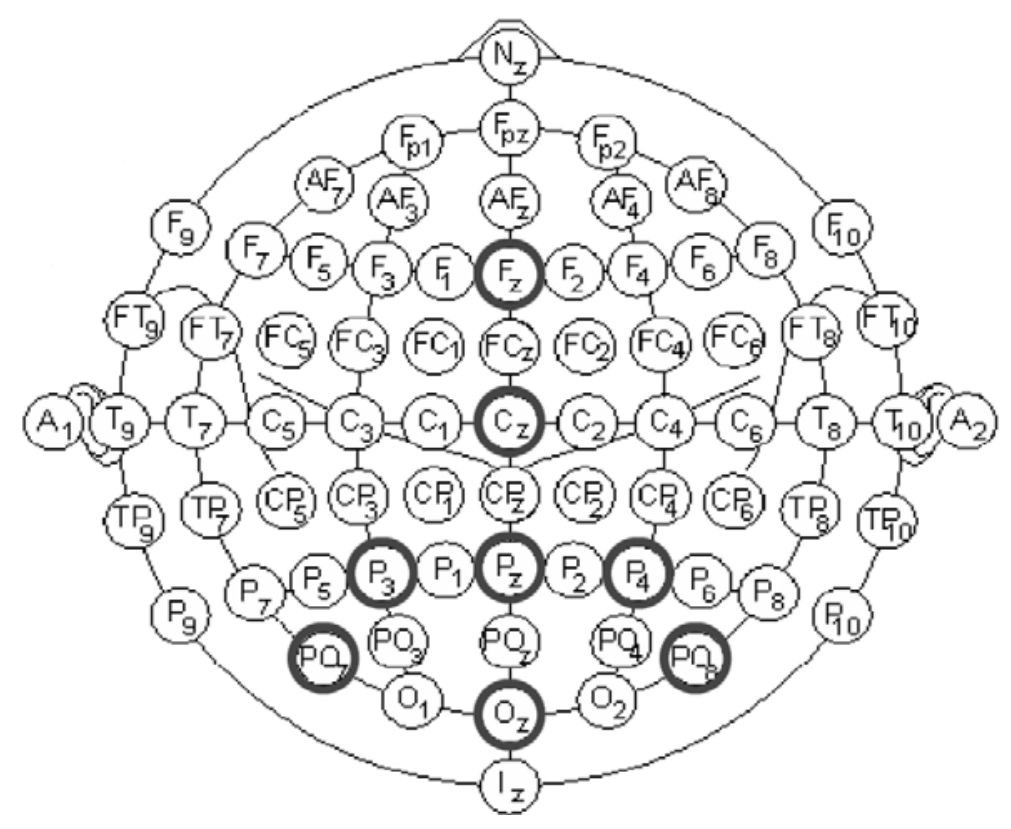

Figure 3-1. EEG recording montage applied in the online sessions.

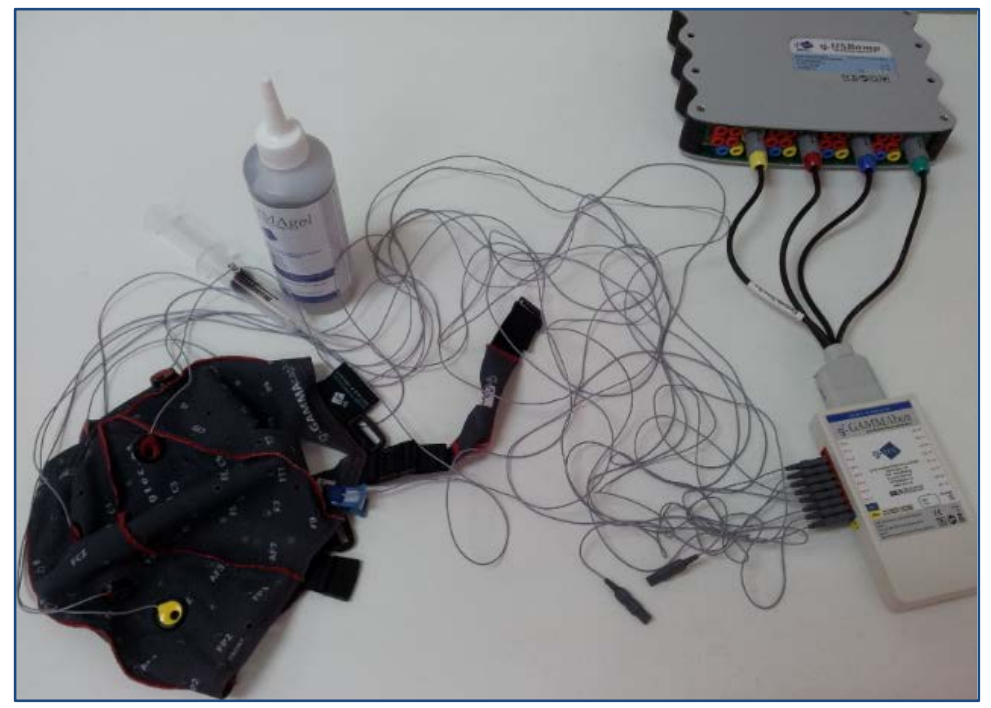

Figure 3-2. EEG recording equipment used in the present study.

the usual RC paradigm, the rows and columns in the matrix flash in turn and randomly at a fast rate, around 4-8 flashes per second. The user can select an item by focusing his attention on it and counting how many times it flashes. The row or column that contains the desired item evokes a P300 response, whereas the others do not, as seen in Figure 33. When all the flashing sequences have finished, the computer can identify the row and column that elicited the evoked potential, and thus the desired item. The involved signal processing is shown in Figure 3-4 and comprises three procedures: feature extraction, offline feature selection and online classification. 


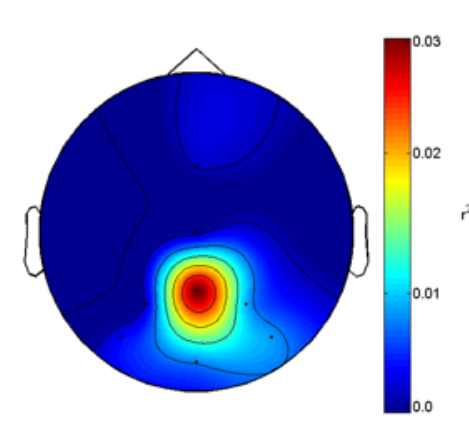

(a)

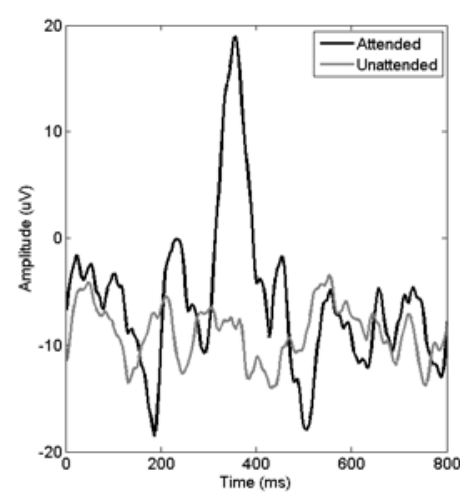

(b)

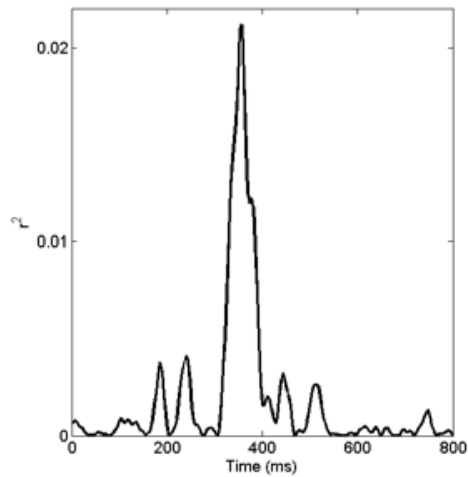

(c)

Figure 3-3. P300 evoked potential. (a) Topographical distribution of the P300 response at 330 ms after stimuli, measured as $\boldsymbol{r}^{2}$ for attended versus unattended items (derived from averaging 15 sequences of stimuli). (b) Time course at $\mathrm{Pz}$ of the voltage for attended (black line) and unattended (grey line) stimuli. (c) Corresponding $\boldsymbol{r}^{2}$ time course that indicates the statistical difference between the attended and unattended items.

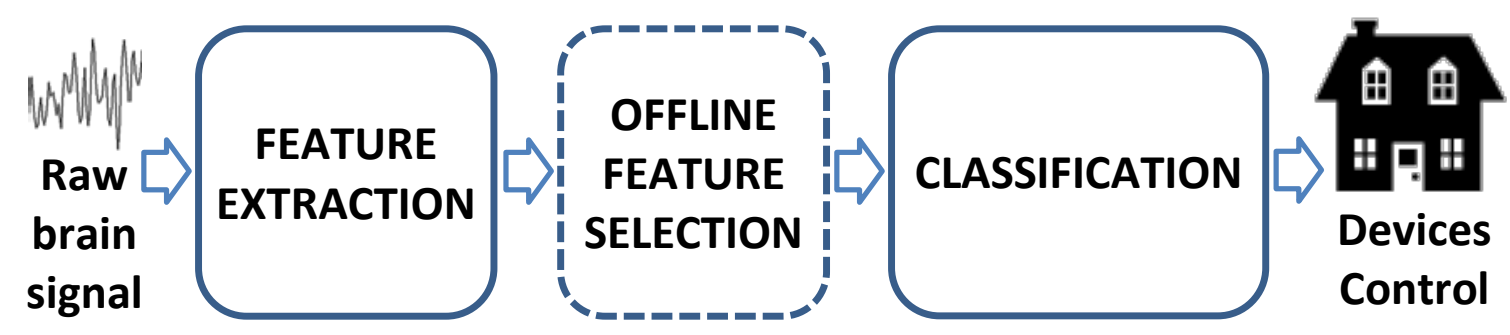

Figure 3-4. EEG signal processing comprises three procedures: feature extraction, offline feature selection and online classification. Once the user's desired option is identified, the selected command is sent to the corresponding device.

Firstly, the feature extraction procedure consists of three stages: signal calibration, spatial filtering and temporal filtering. Secondly, the user performs several runs in copy-spelling mode (Schalk and Mellinger 2010), i.e. the user is told what item has to focus on. Then, these runs are used for the offline feature selection stage: the most suitable features are selected and the custom-built classifier weights are derived for each subject. Finally, an online classifier stage is applied in order to discriminate between the signals that contain the P300 response and the ones that do not. The item in the intersection between the stimuli with the largest probabilities for row and for column is identified as the desired item. The three procedures are exhaustively described in the subsequent sections. 


\subsubsection{Feature Extraction}

The feature extraction procedure consisted of three stages: signal calibration, spatial filtering and temporal filtering. The model is shown in Figure 3-5.

The first stage is the signal calibration. Its purpose is to convert the brain signals that were acquired with the biosignal amplifier into physical units $(\mu \mathrm{V})$. BCI2000 converts raw data into $\mu \mathrm{V}$ using two parameters: a gain factor $g_{c h}$ and an offset factor $o_{c h}$. The last factor has into account any additive signal offset that might be introduced by the acquisition hardware. For a raw signal $r s_{c h}(k)$ acquired at electrode $c h$ and at sample time $k$, the calibrated signal $c s_{c h}(k)$ is derived using a linear equation:

$$
c s_{c h}(k)=\left(r s_{c h}(k)-o_{c h}\right) g_{c h} .
$$

For the present study the recommendations of the g.USBamp amplifier's manufacturer (Guger Technologies 2014) and the BCI2000 developers (Schalk and Mellinger 2010) were taken into account. Therefore, the gain $g_{c h}$ and the offset $o_{c h}$ were set at 1 and 0 , respectively, for the signal calibration stage. Hence, $c s_{c h}(k)=$ $r s_{c h}(k)$.

Once the signal is invariant to changes in the data acquisition hardware, the next stage is the application of a spatial filter. The effect of the distance between the sensors and the signal sources in the brain and the inhomogeneities of the tissues in between causes the spatial noise. Hence, the main purpose of this stage is to sharpen the recorded signals in order to derive a more reliable representation of the sources within the brain, increasing the signal to noise ratio (SNR). Common average reference filters were shown to provide comparable or higher performance than other re-referencing techniques for BCI operation (Alhaddad 2012, Krusienski et al. 2008, McFarland et al. 1997). More specifically, applied to P300 potentials, CAR spatial filtering enhances the

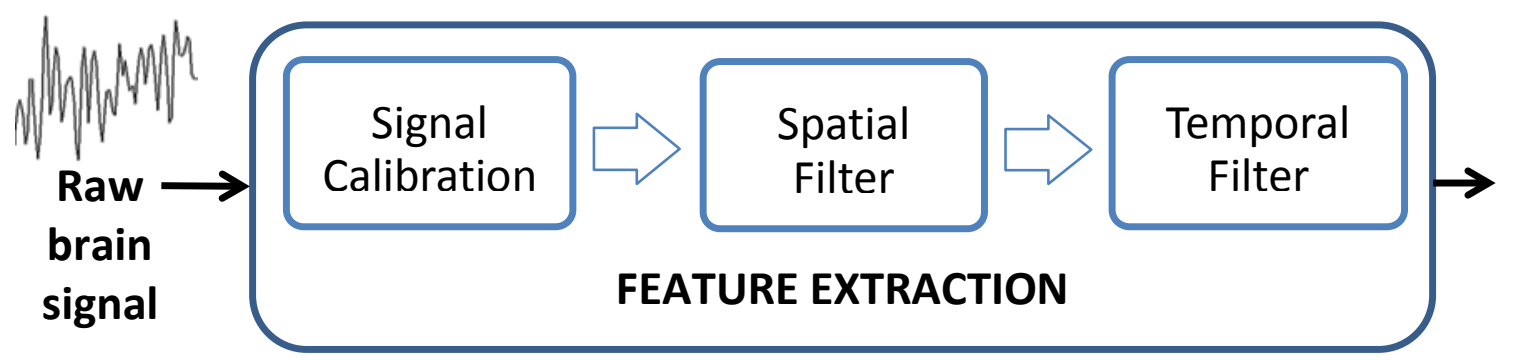

Figure 3-5. Feature extraction procedure consisted of signal calibration, spatial filtering and temporal filtering stages. 
statistical difference $\left(r^{2}\right)$ between the attended and unattended responses, as illustrated in Figure 3-6. For this reason, CAR was applied as spatial filter in the present study. The CAR technique re-references the calibrated signal $c s_{c h}(k)$ of each electrode $c h$ at each sample time $k$ to a reference that is estimated by averaging the signals from all electrodes in the EEG montage $(N)$. Thus, a CAR filter estimates the signal amplitude that is common to all electrodes $\left(\frac{1}{N} \sum_{i=1}^{N} c s_{i}(k)\right)$ and removes it from the signal $c S_{c h}(k)$ at each channel:

$$
s p_{-} c s_{c h}(k)=c s_{c h}(k)-\frac{1}{N} \sum_{i=1}^{N} c s_{i}(k)
$$

The third stage of feature extraction is the application of a temporal filter. In the specific case of P300 evoked potentials the temporal waveforms of the recorded EEG signals are commonly used as signal features (Furdea et al. 2009, Jin et al. 2012, Nijboer et al. 2008, Schalk and Mellinger 2010, Sellers and Donchin 2006). In this study, epochs of data was buffered and averaged as required for online classification of the evoked responses. The epochs of data recorded in response to a number of stimuli are buffered separately for each stimulus. When all the dimming stimuli were done and

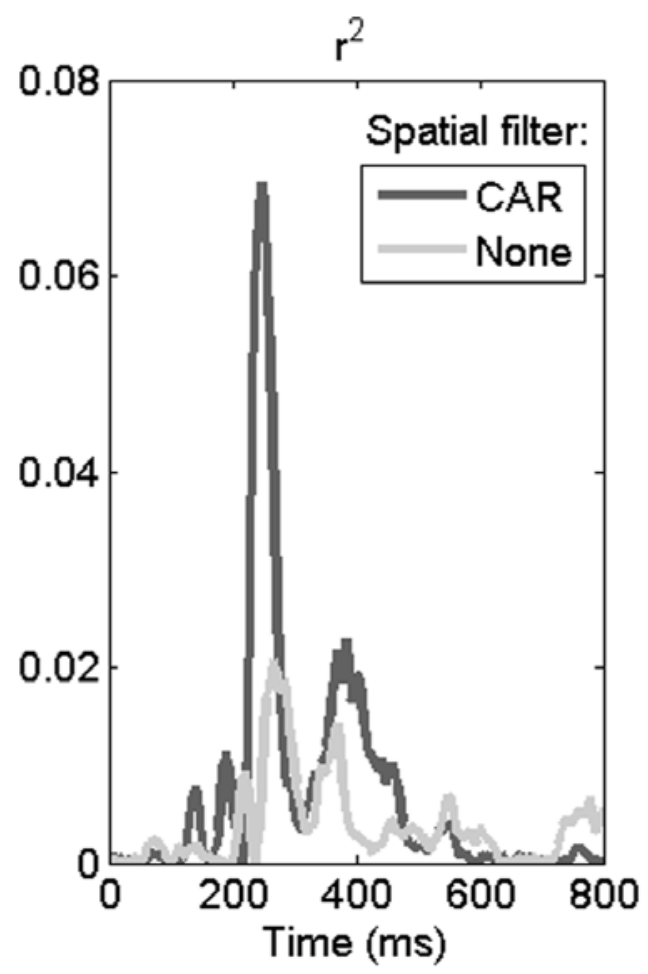

Figure 3-6. Differences in the time course of $\boldsymbol{r}^{\mathbf{2}}$ between not applying any spatial filter and applying CAR to the averaged waveforms. Applied to P300 responses, CAR enhances the statistical difference between the attended and unattended stimuli. 
all the epochs were accumulated for each stimulus, the temporal average is calculated and these averaged waveforms are ready for the online classification procedure (Schalk and Mellinger 2010). In the present study epochs of $800 \mathrm{~ms}$ in length immediately after the beginning of each stimulus were extracted and averaged (Schalk and Mellinger 2010). These epochs should contain the whole evoked responses since P300 potentials appear 300 ms after the stimulus (Wolpaw et al. 2002).

\subsubsection{Feature Selection}

The feature selection procedure is accomplished offline. Each user performed an initial calibration session in copy-spelling mode (Schalk and Mellinger 2010), i.e. the user was told what item (target) had to focus on. Hence, the information about what were the attended and unattended stimuli was available. This initial session was comprised of 10 runs consisted of 5-6 items each. In this study, the feature selection procedure was applied to data from the 5 first runs of the Calibration session in order to determine the classifier weights for each user, similarly to the protocol performed by Mak et al. (2012). Thus, the most suitable features are selected and the custom-built classifier weights for each user are derived.

Stepwise Linear Discriminant Analysis (SWLDA) was applied as feature selection method in the present study. SWLDA selects suitable features to be included in a multiple regression model (Krusienski et al. 2008). For binary classification tasks, such as identifying if a stimulus elicited an ERP or not, the linear discriminant and least-squares regression solutions are equivalent (Schalk and Mellinger 2010). A combination of forward and backward stepwise selection was applied. Sequential forward selection and backward elimination algorithms allow to explore the original space of $p$ features looking for a subset, without calculating all the possible combinations (Sutter and Kalivas 1993). As a result of adding or removing variables sequentially, this methodology provides a group of nested subsets incorporating progressively more and more variables of decreasing relevance (Guyon and Elisseeff 2003, Mao 2002).

The stepwise method decides to add or to remove a feature from the model by means of an iterative process. It selects the strongest features from the feature set and 
removes the features that provide redundant information in terms of statistical significant differences associated with the F-test (Draper and Smith 1981, Schalk and Mellinger 2010). The procedure is described below.

(1) At the first step, the most important feature, i.e.: the one with the smallest $p$-value from the statistical F-test, is added to the model if its associated $p$-value is less than a significance level $p_{\text {in }}$.

(2) At the second step, to determine whether any of the remaining variables are important once this feature is in the model, the algorithm searches for statistical differences among the present model and every new model composed of the current feature and one of the remaining variables. The single feature with the smallest $p$-value is added to the model is its associated $p$-value is less than $p_{\text {in }}$. Now, it is possible that the previously selected feature is no longer important once the current variable has been added to the model. Thus, the next step performs a check for backward elimination (Hosmer and Lemeshow 1989, Sellers and Donchin 2006).

(3) The backward elimination is accomplished by fitting models that delete one of the features added in the previous steps and by assessing the importance of the variable removed. To determine whether a feature should be deleted from the model the algorithm selects that variable which, when removed, yields the maximum $p$-value. If the maximum $p$-value to remove exceeds the significance level $p_{\text {out }}$, then the associated feature is removed from the model. Otherwise, the variable remains in the model. Subsequently, the step 4, a check for forward selection, is performed.

(4) In order to determine whether any of the remaining features are important at this moment, the algorithm searches for statistical differences between the current model and every new model composed of the current variables and one of the remaining features. The single feature with the smallest $p$-value is added to the model is its associated $p$-value is less than $p_{\text {in }}$. Then, the step 3 , a check for backward elimination, is performed again.

The process of backward elimination (step 3) followed by forward selection (step 4) continues in this manner until one of these conditions is fulfilled: (i) all features in the model have $p$-values lower than $p_{\text {out }}$ and the remaining features have $p$-values 
greater than $p_{\text {in }}$ or (ii) the model reaches a maximum predetermined number of features (Hosmer and Lemeshow 1989, Schalk and Mellinger 2010). Therefore, SWLDA performs feature space reduction by selecting suitable spatiotemporal features (i.e., the amplitude value at a particular time sample and channel location) to be included in a linear discriminant function based on the features with the greatest unique variance (Krusienski et al. 2006, Nijboer et al. 2008). In this study, the discriminant function was restricted to contain a maximum of 60 features and it was derived by using $p_{\text {in }}=0.1$ and $p_{\text {out }}=0.15$. It was shown that these are proper values for P300-related studies and, actually, they are commonly applied in P300-based BCIs (Aloise et al. 2011a, Kleih et al. 2010, Krusienski et al. 2006, Krusienski et al. 2008, Nijboer et al. 2008, Schalk and Mellinger 2010, Sellers and Donchin 2006, Sellers et al. 2006). The spatiotemporal features selected in this process compose the specific classifier for each subject, which will be used for managing the assistive BCI tool in the subsequent sessions.

\subsubsection{Feature Classification}

In the previous feature extraction procedure, brain signals were spatially and temporally filtered, resulting in a set of extracted features. Subsequently, the most relevant features were selected. Then, the classifier uses these features to differentiate in real time between signals that contain the P300 response and ones that do not. In BCIs, online P300 detection is typically accomplished by means of traditional classification techniques, such as linear discriminant analysis (Schalk 2006).

In this study, a linear classifier was applied. Other classification methods were also used for offline P300 detection. However, only Krusienski et al. (2006) carried out a comprehensive comparison of different methods applied to the same data set. They showed that stepwise discriminant analysis and a lineal classifier provided the best overall performance and implementation characteristics for practical classification (Fazel-Rezai et al. 2012), when compared to Pearson's correlation method (PCM), a linear support vector machine (LSVM) and a Gaussian kernel support vector machine (GSVM). This indicates that the error, mostly due to lack of P300 in EEG, resulted from factors such as human error, adjacency effect, or mental fatigue (Fazel-Rezai et al. 2012). 
Linear Discriminant Analysis (LDA) finds a linear combination of features which characterizes or separates two or more classes of objects or events. Hence, the linear classifier computes a projection of a high-dimensional signal feature space onto a low-dimensional classification space. Thus, each dimension of classification space is a linear combination of signal features. LDA assumes that the conditional probability density functions are normally distributed for each class. The resulting linear combination may be used as a classifier:

$$
D(k)=\boldsymbol{w}^{T} \boldsymbol{x}(k)+w_{0},
$$

where $\boldsymbol{x}(k)$ is the feature vector at a sample time $k, \boldsymbol{w}$ is the weight vector and $w_{0}$ is a threshold obtained by LDA. In an ERP-based BCI (such as the P300 BCI), the feature vector $\boldsymbol{x}(k)$ is a sequence of averaged EEG time courses obtained in response to a number of stimuli, and the output $D(k)$ is related to the likelihood for the corresponding response to be an ERP (Schalk and Mellinger 2010, Schlögl et al. 1997).

The row or column that contains the target or desired item evokes a P300 response, whereas the others do not, as shown in Figure 3-3. After averaging all stimuli responses, the online classifier takes waveform data from multiple locations and time points to linearly combine them into a single value. Thus, the specific spatiotemporal features that compose the classifier are combined into a single number. Under the assumption that noise is Gaussian distributed with equal covariance for both classes, this value represents the likelihood for each response to be an ERP (Schalk and Mellinger 2010). This study proposes a RC stimuli pattern. Therefore, the item in the intersection of the row and column with the greatest response values will be considered as the desired target. 



\section{4. $\mathrm{BCl}$ tool for managing electronic devices at home}

4.1. Main needs of the potential end-users

4.2. Operation of the application

4.3. Design of the BCI assistive tool

4.3.1. Main menu

4.3.2. TV menu

4.3.3. DVD player menu

4.3.4. Hi-Fi system menu

4.3.5. Multimedia hard drive menu

4.3.6. Lights menu

4.3.7. Heater menu

4.3.8. Fan menu

4.3.9. Phone menu

4.3.10. Phone book menu

4.3.11. BCI2000 and RedRat

4.4. Assessment procedure by means of real end-users

4.4.1. Calibration session

4.4.2. Evaluation 1 session

4.4.3. Evaluation 2 session

4.4.4. Assessment 



\subsection{Main needs of the potential end-users}

In this study, a P300-based BCI tool for managing electronic devices at home was developed. To that purpose, the main needs of the potential end-users of BCI systems were previously analysed.

Many different disorders can disrupt the channels through which the brain normally communicates with and controls its external environment (Wolpaw et al. 2002). ALS, brainstem stroke, brain or spinal cord injury, cerebral palsy, muscular dystrophies, MS and numerous other degenerative diseases impair the neural pathways that control muscles or impair the muscles themselves (Wolpaw et al. 2002 ). BCIs can allow these patients to express their wishes to the outside world (Schalk 2006).

The main needs of potential BCIs end-users were identified with the collaboration of the patients and staff (physiotherapists, neuropsychologists, nurses, assistants, etc.) from the NRC-D\&D. The main requirements were related to comfort, communication and security needs. Table 4-1 summarizes the most common requests of these patients.

The specific requirements were clustered into three main groups: comfort, communication and security related requests. Firstly, patients from the NRC-D\&D identified the comfort-related needs. Their specific requests were divided into three categories: environment, hygiene and mobility. Potential BCIs end-users demanded accessible interfaces for managing the devices usually present at their environment, such as turn on/off lights, turn on/off and adjust the power of heating and air conditioning system, open/close windows, lower/raise roller shutters and activate/deactivate sockets in their own rooms. They also required technical aids for their daily hygiene procedures. Additionally, they indicated mobility needs for managing electric adjustable beds and movement of wheelchairs more easily. Secondly, the patients from the NRC-D\&D identified communication-related needs. These requirements were also related to accessibility and entertainment needs. They demanded simpler interfaces for managing devices associated with communication and entertainment, such as televisions (TV), DVD players, High-Fidelity (Hi-Fi) systems, etc. Moreover, they indicated that easier and more accessible interfaces for computers, Web surfing and e-mailing were needed. Finally, some security-related needs were identified. Telecare systems and fall detectors in the patients' usual environment were 


\begin{tabular}{|c|c|c|}
\hline Needs & \multicolumn{2}{|c|}{ Specific requirements } \\
\hline \multirow{9}{*}{ Comfort } & \multirow{6}{*}{ Environment } & Lights \\
\hline & & Heating \\
\hline & & Air conditioning \\
\hline & & Windows \\
\hline & & Roller shutters \\
\hline & & Sockets \\
\hline & \multicolumn{2}{|l|}{ Hygiene } \\
\hline & \multirow{2}{*}{ Mobility } & Wheelchairs \\
\hline & & Electric beds \\
\hline \multirow{7}{*}{$\begin{array}{l}\text { Communication, } \\
\text { accessibility and } \\
\text { entertainment }\end{array}$} & \multicolumn{2}{|l|}{ Computers } \\
\hline & \multicolumn{2}{|c|}{ Phone and videoconference } \\
\hline & \multicolumn{2}{|c|}{ E-mail } \\
\hline & \multicolumn{2}{|l|}{ TV } \\
\hline & \multicolumn{2}{|l|}{ DVD } \\
\hline & \multicolumn{2}{|l|}{ Hi-Fi systems } \\
\hline & \multicolumn{2}{|c|}{ Internet (Web surfing) } \\
\hline \multirow{3}{*}{ Security } & \multicolumn{2}{|c|}{ Telecare } \\
\hline & \multicolumn{2}{|l|}{ Fall detector } \\
\hline & \multicolumn{2}{|c|}{ Access control } \\
\hline
\end{tabular}

Table 4-1. The most common requirements of potential BCIs end-users from the NRCD\&D.

the main requirements. Also, they included management of access control systems as another security-related need at home.

In the present study, these needs were taken into consideration and the developed assistive BCI tool meets much of the identified needs. Specifically, it is possible to control eight electronic devices usually present at home: TV, DVD player, Hi-Fi system, multimedia drive, lights, heater, fan, and phone. Therefore, the proposed BCI tool satisfies requirements related to comfort, communication, accessibility and entertainment.

\subsection{Operation of the application}

The user's task in the oddball paradigm (Farwell and Donchin 1988, Hillyard and Kutas 1983, Pritchard 1981) requires to determine to which of two possible categories each item in a series belongs when one of these categories occurs 
rarely. These infrequent items elicit an ERP with an enhanced component with a latency of about 300 ms, called the P300 (Farwell and Donchin 1988).

The assistive BCI tool proposed in the present study is based on the oddball paradigm which evokes a P300 response to rare stimuli when they are interspersed with frequent stimuli. The proposed application allows the users to control several devices related to comfort, communication and entertainment needs at home. Instead of the single matrix consisted of characters presented in the typical paradigm (Donchin et al. 2000, Farwell and Donchin 1988, Nijboer et al. 2008), the proposed application shows the user several matrices of pictures. Specifically, the assistive tool consists of a main menu and nine sub-menus. Each menu presents a matrix of arranged images depicting the electronic devices or their specific control commands. The typical P300 paradigm uses a RC pattern approach of flashing stimuli (Donchin et al. 2000, Farwell and Donchin 1988, Nijboer et al. 2008). The developed assistive tool proposes the same RC pattern approach using dimming stimuli. It was experimentally noticed that dimming stimuli were more prominent and users reported that visual annoyance was reduced for these specific pictures (Corralejo et al. 2014).

At the beginning, the main menu of the assistive BCI tool is presented. Figure 4-1(a) shows the main menu, which consists of a 3 x 4 matrix of pictures. Each item in the first two rows of the matrix depicts one device: TV, DVD player, Hi-Fi system, multimedia drive, lights, heater, fan and phone. The last row shows the phone book and three control commands: pause, resume and stop the application. According to the typical P300 paradigm (Donchin et al. 2000), 15 sequences of random stimuli are presented for each item selection. Each sequence contains one dimming stimulus for each row and for each column (i.e., 7 stimuli for the main menu). Figure 4-1(b) shows the main menu while the third column is dimmed. Stimuli occur randomly every 187.5 ms: each stimulus dims for $62.5 \mathrm{~ms}$ and then the screen remains unvarying for $125 \mathrm{~ms}$ (Furdea et al. 2009, Kleih et al. 2010, Spüler et al. 2012). Users are asked to focus their attention to a specific item from the matrix and count how many times it dims. The duration of the stimuli and the pauses were chosen to allow our population of potential end-users to properly note the stimuli and thus to suitably count them. Then, once the matrix finishes flickering, auditory feedback is given and the selected command is performed: accessing a specific sub-menu or pause, resume or stop the application. Thus, users can access several sub-menus for managing a specific device from the main 


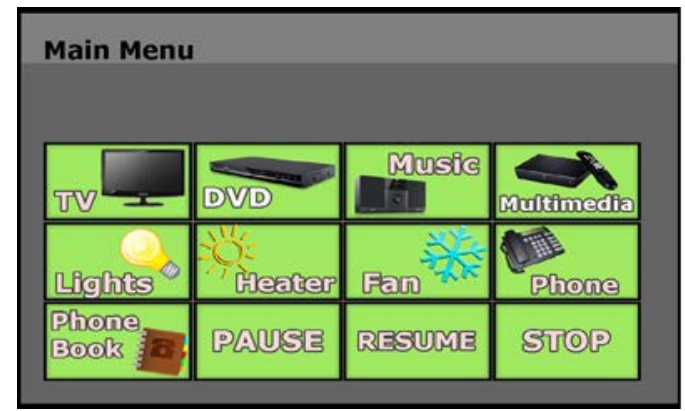

(a)

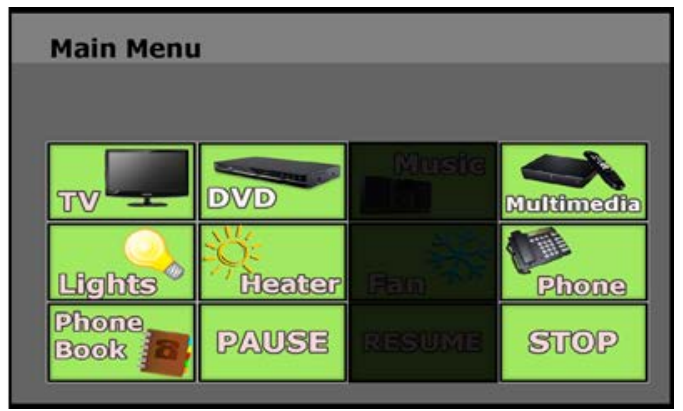

(b)

Figure 4-1. (a) Main menu of the assistive BCI tool. It shows a $3 \times 4$ matrix consisted of images depicting all available devices: TV, DVD player, Hi-Fi system, multimedia drive, lights, heater, fan, and phone. The phone book was included as an independent option in the main menu. Thus, users are able to access from the main menu to a specific sub-menu of each device and to pause, resume or stop the run. (b) Main menu while the third column is dimmed.

menu. Sub-menus are implemented by means of variable size matrices, consisted of images depicting the specific commands of each device and the option of returning to the main menu. Sizes of sub-menus matrices are $3 \times 4,4 \times 4$ or $5 \times 5$, depending on the functionalities of each device. All sub-menus are thoroughly described in the subsequent subsections. Likewise to the main menu, rows and columns of the sub-menu matrix are randomly dimmed. Users are asked again to attend to the item depicting the desired command and silently count how many times it dims. Once the system identifies the desired option, auditory feedback is given and the appropriate control command is performed (e.g., switch off the fan or turn the TV volume up). Then, a pause of $6 \mathrm{~s}$ is set in order to satisfy two requirements: (i) users are able to check if the desired menu was accessed or the desired command was properly performed, and (ii) users can also locate the next required item within the matrix. Therefore, users navigate through the developed tool by means of multiple menus and operate eight electronic devices, which are usually present at home.

\subsection{Design of the BCI assistive tool}

The proposed assistive BCI application allows users to control 8 electronic devices and up to 115 control commands related to comfort, communication and entertainment needs. Specifically, the developed assistive tool manages the following 
devices and their main functionalities: TV, DVD player, Hi-Fi system, multimedia hard drive, lights, heater, fan and phone. The real devices used in the present study are shown in Figure 4-2.

This section describes the assistive BCI tool's graphical design: the main menu and the sub-menus as well as all the available commands. The specific sub-menus are implemented by means of variable size matrices, consisted of several images depicting different commands of each device and the option of returning to the main menu. All images belonged to a specific sub-menu have the same background colour. Thus, it is easier to identify each sub-menu for the end-user. Moreover, items belonged to each sub-menu were distributed in the matrix simulating the distribution of the real remote control for the corresponding device. The control commands included in each matrix were selected for the specific model and functions of each device used in this study.

\subsubsection{Main menu}

Initially, the main menu of the assistive BCI tool is presented. As shown in Figure 4-1, it consists in a 3 x 4 matrix of pictures with light green background. Each item in the first two rows of the matrix depicts one specific device: TV, DVD player,

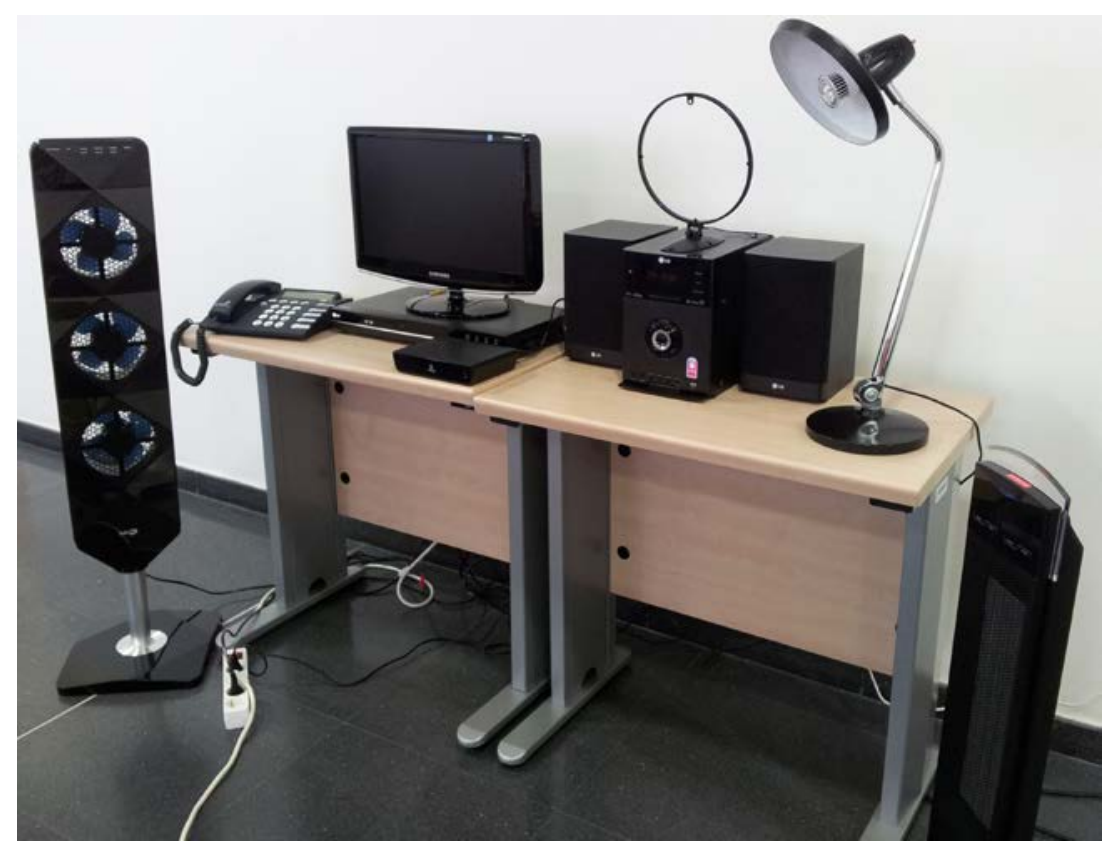

Figure 4-2. Picture of the eight real devices that the user can manage by means of the proposed BCI tool. 
Hi-Fi system, multimedia drive, lights, heater, fan and phone. The last row shows the phone book and three control commands: pause, resume and stop the application. Hence, from the main menu users can access the different sub-menus for managing a specific device.

The items that can be selected from the main menu and their specific functions are described below:

- TV: access the television control menu.

- DVD: access the DVD player control menu.

- Music: access the Hi-Fi system control menu.

- Multimedia: access the multimedia hard drive control menu.

- Lights: access the lights control menu.

- Heater: access the heater control menu.

- Fan: access the fan control menu.

- Phone: access the telephone control menu.

- Phone book: access the phone book menu. This menu is also reachable from the phone control menu.

- Pause: suspend/pause the application execution. Once the application is paused, rows and columns continue dimming. Then, at any time the user can focus on an item and try to select it. However, when the execution is paused only the 'Resume' item can be effectively selected in order to restart the application execution.

- Resume: restart the application execution when it was previously paused.

- Stop: stop and end the application running.

\subsubsection{TV menu}

The TV control menu consists on a 5 x 5 matrix of images (red background) depicting the main commands for a TV. From this menu, users are able to operate the TV likewise using the typical TV remote control. The specific TV model used in this 
study was: SAMSUNG SyncMaster 933HD. The TV menu is shown in Figure 4-3. The commands that can be selected from this menu are described below.

- ON/OFF: switch the TV on (if switched off) or switch it off (if switched on).

- Channel up: skip to the next TV channel.

- Channel down: skip to the previous TV channel.

- 0-9: select a specific channel sending numbers from 0 to 9.

- Volume up: turn the TV volume up.

- Volume down: turn the TV volume down.

- MUTE: mute the TV volume.

- MENU: access the TV menu configuration.

- ENTER: select the current option inside the TV menu configuration.

- EXIT: leave the TV menu configuration.

- Leftwards arrow: move left inside the TV menu configuration.

- Rightwards arrow: move right inside the TV menu configuration.

- Upwards arrow: move up inside the TV menu configuration.

\begin{tabular}{|c|c|c|c|c|}
\hline $\begin{array}{l}\text { ON/ } \\
\text { OFF }\end{array}$ & channel & 1 & 2 & 3 \\
\hline volume & |channel & 4 & 5 & 6 \\
\hline volume & 4 & 7 & 8 & Q) \\
\hline$<$ & MENU & & 0 & $\begin{array}{l}\text { Program } \\
\text { Guide }\end{array}$ \\
\hline ENTER & $Y$ & EXI & प्र & $\begin{array}{c}\text { RETURN } \\
\text { to main } \\
\text { menu }\end{array}$ \\
\hline
\end{tabular}

Figure 4-3. TV menu. It shows a 5 x 5 matrix consisted of images depicting the main TV commands: switch on/off, skip to a specific TV channel, turn up/down or mute the sound, access and navigate TV menu, access the program guide and return to the main menu. From this menu, users are able to operate the TV as using a real remote control. 
- Downwards arrow: move down inside the TV menu configuration.

- Program Guide: access the TV Episode Guide Program (EPG).

- RETURN to main menu: come back to the main menu.

\subsubsection{DVD player menu}

The DVD player control menu consists on a $3 \times 4$ matrix of images (blue background) depicting the main commands for a DVD player. Figure 4-4(a) shows the DVD player control menu. The specific DVD player used in the present study was: LG DR389. The available control commands for the DVD player menu are detailed below.

- ON/OFF: switch the DVD player on (if switched off) or switch it off (if switched on).

- Play: start or resume watching a video.

- Pause: make a pause watching a video.

- Stop: finish/stop watching a video.

- Leftwards paired arrows (backward): skip to the previous video track.

- Rightwards paired arrows (forward): skip to the next video track.

- Menu/List: explore the DVD's content.

- REC: record the current channel from the TV.

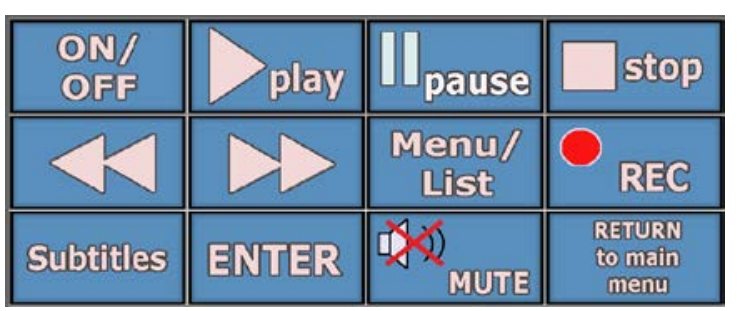

(a)

\begin{tabular}{|c|c|c|c|}
\hline $\begin{array}{l}\text { (0) } \\
\text { (1) } B 53\end{array}$ & volume S & 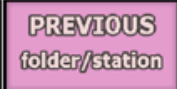 & \begin{tabular}{|c|} 
NESXS \\
colder/startion
\end{tabular} \\
\hline $\begin{array}{l}\text { PLAYO } \\
\text { PAUSES }\end{array}$ & nolume 3 & $\begin{array}{l}</ \\
\text { backwward }\end{array}$ & forward \\
\hline ST(0) & पर X) & Radio / GD & 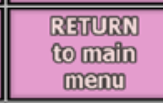 \\
\hline
\end{tabular}

(b)

Figure 4-4. (a) DVD menu. It presents a 3 x 4 matrix consisted of images depicting the main DVD commands: switch on/off, play, pause or stop a video, explore contents, skip to the next or previous file, mute, record the actual channel and return to the main menu. (b) Music menu. It shows a $3 \times 4$ matrix consisted of images depicting the main musicrelated commands: switch on/off, volume control, skip to radio or CD function, play, pause or stop a track, skip to the next or previous track or radio station and return to the main menu. 
- Subtitles: show (if hidden) or hide (if showed) the subtitles for a video.

- ENTER: select a specific file from the menu/list of DVD’s content.

- MUTE: mute the TV volume.

- RETURN to main menu: come back to the main menu.

\subsubsection{Hi-Fi system menu}

The Music menu consists on a 3 x 4 matrix of images (lilac background) depicting the main commands for controlling the Hi-Fi system. The music menu is shown in Figure 4-4(b). The specific Micro Hi-Fi system used in this study was: LG XA63. The commands that can be selected from this menu are described below.

- ON/OFF: switch the Hi-Fi system on (if switched off) or switch it off (if switched on).

- PLAY/PAUSE: start/resume or pause the playing of a CD track.

- STOP: finish/stop playing a CD track.

- Volume up: turn the Hi-Fi system volume up.

- Volume down: turn the Hi-Fi system volume down.

- MUTE: mute the Hi-Fi system volume.

- Next Folder/Station: skip to the next folder or radio station.

- Previous Folder/Station: skip to the previous folder or radio station.

- Leftwards paired arrows (backward): skip to the previous CD track.

- Rightwards paired arrows (forward): skip to the next CD track.

- Radio/CD: skip to the radio function (when CD is actually selected) or CD function (when radio is actually selected).

- RETURN to main menu: come back to the main menu. 


\subsubsection{Multimedia hard drive menu}

The Multimedia menu consists on a $3 \times 4$ matrix of images (purple background) depicting the main commands for a multimedia hard drive. Figure 4-5(a) shows the Multimedia drive menu. The specific multimedia hard drive used in the present study was: Iomega ScreenPlay MX Full HD 1TB HDMI. The available control commands for the multimedia menu are detailed below.

- ON/OFF: switch the multimedia hard drive on (if switched off) or switch it off (if switched on).

- MENU: explore the multimedia drive's content.

- Subtitles: show (if hidden) or hide (if showed) the subtitles for a video.

- STOP: finish/stop watching a video.

- ENTER/PLAY/PAUSE: select a specific file from the menu of multimedia drive's contents or start, resume or pause a video or audio track.

- Leftwards arrow: move left inside the menu of multimedia drive's content.

- Rightwards arrow: move right inside the menu of multimedia drive's content.

- Upwards arrow: move up inside the menu of multimedia drive’s content.

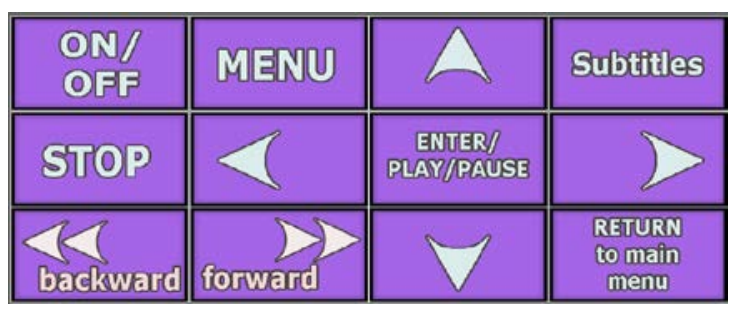

(a)

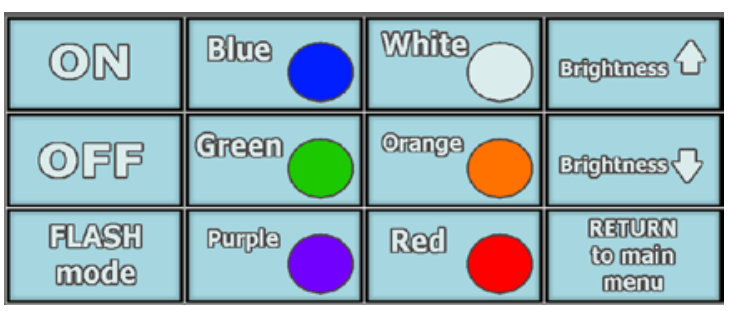

(b)

Figure 4-5. (a) Multimedia menu. It shows a 3 x 4 matrix consisted of images depicting the main DVD commands: switch on/off, explore hard drive's contents, play, pause, stop or skip to the next or previous file, show or hide subtitles and return to the main menu. (b) Lights menu. It presents a $3 \times 4$ matrix consisted of images depicting the main music-related commands: switch on/off, change the light colour, turn up/down the light intensity, enable the flashing mode and return to the main menu. 
- Downwards arrow: move down inside the menu of multimedia drive's content.

- Leftwards paired arrows (backward): skip to the previous file.

- Rightwards paired arrows (forward): skip to the next file.

- RETURN to main menu: come back to the main menu.

\subsubsection{Lights menu}

The Lights control menu consists on a 3 x 4 matrix of images (light blue background) depicting the main commands for managing a LED light bulb at home. The lights menu is shown in Figure 4-5(b). The specific LED lamp used in this study was: Microluz ML-RCLL5W. The commands that can be selected from this menu are described below.

- ON: switch the light on (if switched off).

- OFF: switch the light off (if switched on).

- FLASH mode: activate the flashing mode (i.e., the colour of the light changes every few seconds).

- Blue: set the light colour to blue.

- Green: set the light colour to green.

- Purple: set the light colour to purple.

- White: set the light colour to white.

- Orange: set the light colour to orange.

- Red: set the light colour to red.

- Brightness up: increase the light brightness.

- Brightness down: decrease the light brightness.

- RETURN to main menu: come back to the main menu. 


\subsubsection{Heater menu}

The heater control menu consists on a $3 \times 4$ matrix of images (brown background) depicting the main commands for a heater. Figure 4-6(a) shows the heater control menu. The specific heater used in the present study was: Saivod TW 2500W SLIM. The available control commands for the heater menu are detailed below.

- ON/OFF: switch the heater on (if switched off) or switch it off (if switched on).

- Power up/down: increase or decrease the power intensity of the heater.

- SWING: enable/disable the swing function for the heater.

- TIMER 30 min: program the sleep function for 30 minutes.

- TIMER 1 h: program the sleep function for 1 hour.

- TIMER 1h 30min: program the sleep function for 1 hour and 30 minutes.

- TIMER 2 h: program the sleep function for 2 hours.

- TIMER 2h 30min: program the sleep function for 2 hours and 30 minutes.

- TIMER 3 h: program the sleep function for 3 hours.

- TIMER 3h 30min: program the sleep function for 3 hours and 30 minutes.

\begin{tabular}{|c|c|c|c|}
\hline $\begin{array}{l}\text { ON/ } \\
\text { OFF }\end{array}$ & Power & SWING & $\begin{array}{l}\text { TIMER } \\
30 \mathrm{~min}\end{array}$ \\
\hline $\begin{array}{l}\text { UIMER } \\
\qquad \mathrm{h}\end{array}$ & $\begin{array}{l}\text { UIMER } \\
\text { IT } 30 \mathrm{~min}\end{array}$ & $\begin{array}{l}\text { TIMER } \\
2 \mathrm{~h}\end{array}$ & $\begin{array}{l}\text { TIMER } \\
2 \text { h } 30 \mathrm{~min}\end{array}$ \\
\hline $\begin{array}{l}\text { TIMER } \\
3 \mathrm{~h}\end{array}$ & $\begin{array}{l}\text { TLMER } \\
3 \mathrm{~h} 30 \mathrm{~min}\end{array}$ & $\begin{array}{l}\text { TIMER } \\
4 \mathrm{~h}\end{array}$ & $\begin{array}{l}\text { RETURN } \\
\text { to main } \\
\text { menu }\end{array}$ \\
\hline
\end{tabular}

(a)

\begin{tabular}{|c|c|c|c|}
\hline $\begin{array}{l}\text { OND } \\
\text { POWER }\end{array}$ & (0) 际 & $\begin{array}{c}\text { Infernal Fan } \\
\text { 1. }\end{array}$ & MODE \\
\hline $\begin{array}{l}\text { TUMER } \\
\text { 30minh }\end{array}$ & $\begin{array}{l}\text { UIMERB } \\
\sqrt{16}\end{array}$ & \begin{tabular}{|c|} 
Infernal Fan \\
2
\end{tabular} & SWWA \\
\hline $\begin{array}{l}\text { TEMER } \\
26 \mathrm{~b}\end{array}$ & $\begin{array}{l}\text { TELERR } \\
3 \mathrm{~h}\end{array}$ & \begin{tabular}{|c} 
Infernalfan \\
3
\end{tabular} & $\begin{array}{l}\text { RETUURM } \\
\text { to maflo } \\
\text { meno }\end{array}$ \\
\hline
\end{tabular}

(b)

Figure 4-6. (a) Heater menu. It presents a 3 x 4 matrix consisted of images depicting the main DVD commands: switch on/off, turn up/down power intensity, program the sleep function from 30 min to 4 hours, enable or disable the swing mode and return to the main menu. (b) Fan menu. It shows a $3 \times 4$ matrix consisted of images depicting the main music-related commands: switch on/off, increase or decrease speed, program the sleep function, enable or disable a specific internal fan or the swing mode and return to the main menu. 
- TIMER 4 h: program the sleep function for 4 hours.

- RETURN to main menu: come back to the main menu.

\subsubsection{Fan menu}

The fan control menu consists on a $3 \times 4$ matrix of images (orange background) depicting the main commands for controlling a fan. The fan menu is shown in Figure 4-6(b). The specific fan device used in this study was: Saivod VT-90 Slim. This fan is composed of 3 internal fans, thus some control commands in the menu are specific for controlling these internal fans. The commands that can be selected from this menu are described below.

- ON/POWER: switch the fan on (if switched off) or change the speed of the currently enabled internal fans.

- OFF: switch the fan off (if switched on).

- Internal Fan 1: switch the internal fan 1 on (if switched off) or switch it off (if switched on).

- Internal Fan 2: switch the internal fan 2 on (if switched off) or switch it off (if switched on).

- Internal Fan 3: switch the internal fan 3 on (if switched off) or switch it off (if switched on).

- MODE: skip to normal or quiet mode.

- SWING: enable/disable the swing function for the fan.

- TIMER 30 min: program the sleep function for 30 minutes.

- TIMER 1 h: program the sleep function for 1 hour.

- TIMER 2 h: program the sleep function for 2 hours.

- TIMER 3 h: program the sleep function for 3 hours.

- RETURN to main menu: come back to the main menu. 


\subsubsection{Phone menu}

The phone control menu consists on a 4 x 4 matrix of images (yellow background) depicting the main commands for a usual phone. Figure 4-7 shows the phone control menu. The phone used in the present study can be managed by IR commands, the specific model was: SiCare IR Quickphone. The available control commands for the phone menu are detailed below.

- HANG UP/PICK UP: hang up the phone (if picked up) or pick up the phone (if hung up).

- 0-9: dial a digit from 0 to 9.

- Phone book: access the phone book menu. This menu is also reachable from the main menu.

- \#: dial the number sign or hash symbol, used for special phone's functions.

- 112 Emergency phone: automatically dial the emergency (ER) phone number.

- $\mathrm{R}$ recall: redial the last phone number.

- RETURN to main menu: come back to the main menu.

\begin{tabular}{|c|c|c|c|}
\hline $\begin{array}{l}\text { HANA UP/ } \\
\text { PISBS UPP }\end{array}$ & 5 & 23 & 5 \\
\hline $\begin{array}{l}\text { Phone } \\
\text { Bools as }\end{array}$ & $\Delta$ & 5 & (5) \\
\hline كُ & Z & 8 & (9) \\
\hline 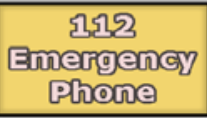 & Rin & (0) & $\begin{array}{c}\text { REVURTN } \\
\text { ç main } \\
\text { mewo }\end{array}$ \\
\hline
\end{tabular}

Figure 4-7. Phone menu. It shows a 4 x 4 matrix consisted of images depicting the main phone control commands: pick up or hang up the phone, dial a phone number, automatically dial the emergency phone number, redial the last phone number, go to the phone book menu for dialling a previously memorized phone number and return to the main menu. 


\subsubsection{Phone book menu}

Finally, the phone book menu consists on a 3 x 4 matrix of images (green background) depicting the main commands related to the phone book. The phone book menu is shown in Figure 4-8. The commands that can be selected from this menu are described below.

- PETER: automatically call Peter (to the phone number for Peter memorized previously).

- JOHN: automatically call John (to the phone number for John memorized previously).

- LISA: automatically call Lisa (to the phone number for Lisa memorized previously).

- DAVID: automatically call David (to the phone number for David memorized previously).

- MARY: automatically call Mary (to the phone number for Mary memorized previously).

- HELEN: automatically call Helen (to the phone number for Helen memorized previously).

- BEN: automatically call Ben (to the phone number for Ben memorized previously).

- WILL: automatically call Will (to the phone number for Will memorized previously).

- SARAH: automatically call Sarah (to the phone number for Sarah

\begin{tabular}{|c||c||c||c|}
\hline PEIER & JOMN & LISA & DAVID \\
\hline \hline MARY & HELEN & BEN & WILL \\
\hline SARAM & $\begin{array}{c}1112 \\
\text { EmergenGy } \\
\text { Phone }\end{array}$ & HANG UP & $\begin{array}{c}\text { REUURN } \\
\text { To main } \\
\text { menu }\end{array}$ \\
\hline
\end{tabular}

Figure 4-8. Phone book menu. It presents a 3 x 4 matrix consisted of images depicting the main phone book commands: nine names of people for automatically dial, which phone numbers were previously memorized, automatically dial the emergency phone number, hang up the phone and return to the main menu. 
memorized previously).

- 112 Emergency Phone: automatically dial the ER phone number.

- HANG UP: hang up the phone for finishing a call.

- RETURN to main menu: come back to the main menu.

\subsubsection{BCI2000 and RedRat}

EEG signal recording and processing stages were detailed in the previous chapter. The BCI2000 configuration parameters for those stages are located in the Filtering and Source configuration tabs of BCI2000 (Schalk and Mellinger 2010). The main parameters applied in this work related to signal recording and processing are shown below.

\begin{tabular}{|c|c|}
\hline$\underline{\text { Parameter Name }}$ & Description and Assigned Value \\
\hline SpatialFilterType & $\begin{array}{l}\text { Type of spatial filter } \\
\text { common average reference CAR }\end{array}$ \\
\hline Epoch length & $\begin{array}{l}\text { Length of data epoch from stimulus onset } \\
800 \mathrm{~ms}\end{array}$ \\
\hline SourceCh & $\begin{array}{l}\text { Number of digitized and stored channels } \\
8\end{array}$ \\
\hline SamplingRate & $\begin{array}{l}\text { Sample rate } \\
256\end{array}$ \\
\hline ChannelNames & $\begin{array}{l}\text { Names of the EEG channels } \\
\text { Fz Cz P3 Pz P4 PO7 PO8 Oz }\end{array}$ \\
\hline SourceChOffset & $\begin{array}{l}\text { Offset for channels in A/D units } \\
00000000\end{array}$ \\
\hline SourceChGain & $\begin{array}{l}\text { Gain for each channel (A/D units -> } \mu \mathrm{V} \text { ) } \\
11111111\end{array}$ \\
\hline FilterEnabled & $\begin{array}{l}\text { Enable band-pass filter ( } 0=\text { no, } 1=\text { yes }) \\
1\end{array}$ \\
\hline FilterHighPass & $\begin{array}{l}\text { High cut-off frequency for band-pass filter } \\
0.1\end{array}$ \\
\hline FilterLowPass & $\begin{array}{l}\text { Low cut-off frequency for band-pass filter } \\
60\end{array}$ \\
\hline AcquisitionMode & $\begin{array}{l}\text { Data acquisition mode (gUSBamp) } \\
\text { analog signal acquisition }\end{array}$ \\
\hline
\end{tabular}


Regarding the development of the assistive BCI tool, it is based on the P3Speller application from the BCI2000 general-purpose system (Schalk et al. 2004, Schalk and Mellinger 2010). As it was previously mentioned, for the present BCI application items were shown as pictures instead of the typical characters or words. Additionally, dimming stimuli were applied since it was larger and less annoying for end-users than flashing stimuli. Therefore, the related BCI2000 parameters TargetDefinitions, IconHighlightMode and IconHighlightFactor were adjusted accordingly (Schalk and Mellinger 2010). Moreover, in order to let users know the specific menu they are watching, a text field was included at the top of the window showing the name of the current menu: "Main Menu”, "TV menu”, "DVD menu”, etc. It can be seen in Figure 4-1 for the main menu of the assistive BCI tool. Thus, three specific parameters (MenuFieldHeight, TextHeightMenuField, and MatrixHeight) were created in order to set the size of this text box, the height of the text inside and the size of the stimuli matrix, respectively. The parameters related to the application appearance and operation are located in the Application configuration tab of BCI2000 (Schalk and Mellinger 2010). The most relevant are presented below.

\begin{tabular}{|c|c|}
\hline Parameter Name & Description and Assigned Value \\
\hline PostSequenceDuration & $\begin{array}{l}\text { Pause following each sequence of intensifications } \\
6 s\end{array}$ \\
\hline StimulusDuration & $\begin{array}{l}\text { Stimulus duration } \\
62.5 \mathrm{~ms}\end{array}$ \\
\hline ISIMinDuration & $\begin{array}{l}\text { Minimum duration of the inter-stimulus interval } \\
125 \mathrm{~ms}\end{array}$ \\
\hline ISIMaxDuration & $\begin{array}{l}\text { Maximum duration of the inter-stimulus interval } \\
125 \mathrm{~ms}\end{array}$ \\
\hline MenuFieldHeight & $\begin{array}{l}\text { Height of the field containing the name of the current menu } \\
0.1\end{array}$ \\
\hline TextHeightMenuField & $\begin{array}{l}\text { Text height for the menu text-field } \\
0.05\end{array}$ \\
\hline MatrixHeight & $\begin{array}{l}\text { Proportional size of the stimuli matrix } \\
1\end{array}$ \\
\hline NumMatrixColumns & $\begin{array}{l}\text { Display matrices' column numbers } \\
4544444444\end{array}$ \\
\hline NumMatrixRows & $\begin{array}{l}\text { Display matrices’ row numbers } \\
3533333343\end{array}$ \\
\hline Language & $\begin{array}{l}\text { Language for user messages } \\
\text { Spanish }\end{array}$ \\
\hline
\end{tabular}


In regard to the management of device's commands, all devices were operated by means of an infrared (IR) emitter device RedRat3-II (RedRat Ltd., Cambridge, UK) connected to the computer. Specifications can be found in the RedRat website (RedRat 2013). The proposed BCI application uses the RedRat Software Development Kit (SDK) for storing and sending the control commands of each device. An IR signals database file, which arranges the IR commands for each device, was created. Figure 4-9 presents the IR signals database structure. Then, the BCI tool takes the command associated with the selected option and sends it to the corresponding device. A specific function for the IR emitter device management was implemented into the application code. This function creates an instance of the class ISignalDB (IR signal database) and opens it. Next, the desired command is identified by means of the variables gDomoMenu (number that identifies the actual menu) and action (number that identifies the selected item inside the actual menu). Then, the RedRat device looks for the corresponding command at the IR signal database and sends it to the specific device.

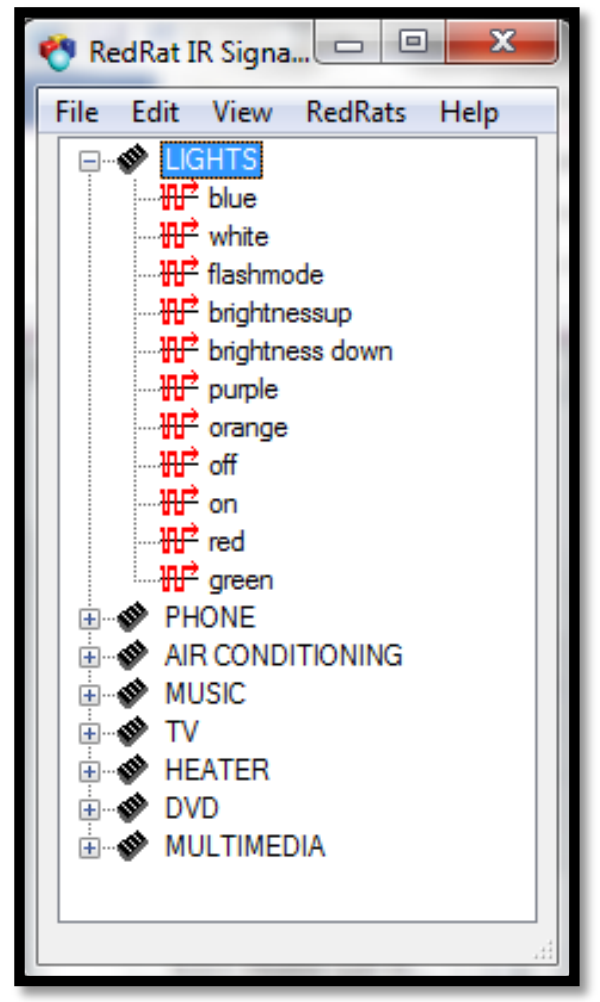

Figure 4-9. IR signal database created for managing electronic devices at home (bd_devices.xml). The database is divided into 8 devices (TV, DVD player, heater, air conditioning, Hi-Fi system, multimedia drive, lights and phone), which contain their specifics commands. In the picture the commands list used for lights control is expanded. 
The assistive BCI tool and the IR control interface were developed using $\mathrm{C}++$ programming language, and the Borland $\mathrm{C}++$ Builder ${ }^{\circledR} 6$ and Microsoft ${ }^{\circledR}$ Visual Studio ${ }^{\circledR} 2010$ Professional development environments.

\subsection{Assessment procedure by means of real end-users}

The assessment procedure was performed by means of real BCI end-users, 30 patients from the NRC-D\&D with disabilities, in a real scenario: real devices in their usual environment. Before the experimental stage, participants were assessed by the neuropsychologists of the NRC-D\&D. Motor and cognitive impairment degrees and sustained attention ability were collected for each subject. Furthermore, participants recruited at the second stage, P16 to P30, also performed the Luria Adult Neuropsychological Diagnosis (AND) test (Christensen 1987). Luria AND includes nine specific sub-tests for assessing nine functions distributed among five different areas: visuospatial (visual perception and spatial orientation), oral language (receptive speech and expressive speech), memory (immediate memory and logical memorization), intelligence (thematic draws and conceptual activity) and attention (attentional control).

The assistive BCI tool was assessed by impaired subjects through three experimental sessions performed at the NRC-D\&D facilities. Participants were seated in their own wheelchair or in a comfortable chair facing a computer flat screen, as Figure 4-10 shows. Each subject was intended to perform three sessions: one session comprised of calibration tasks and two evaluation sessions for assessing their performance with the designed BCI tool (Eval1 and Eval2).

\subsubsection{Calibration session}

The Calibration session approximately lasted one hour. In this initial session, only one matrix was presented to the user: the 5 x 5 TV menu matrix. Data was collected in copy-spelling mode (Krusienski et al. 2008, Sellers et al. 2006). Calibration session was comprised of 10 runs of approximately $4 \mathrm{~min}$. For each run, the user was asked to attend a specific item from a proposed series of 5-6 items. 15 sequences of 


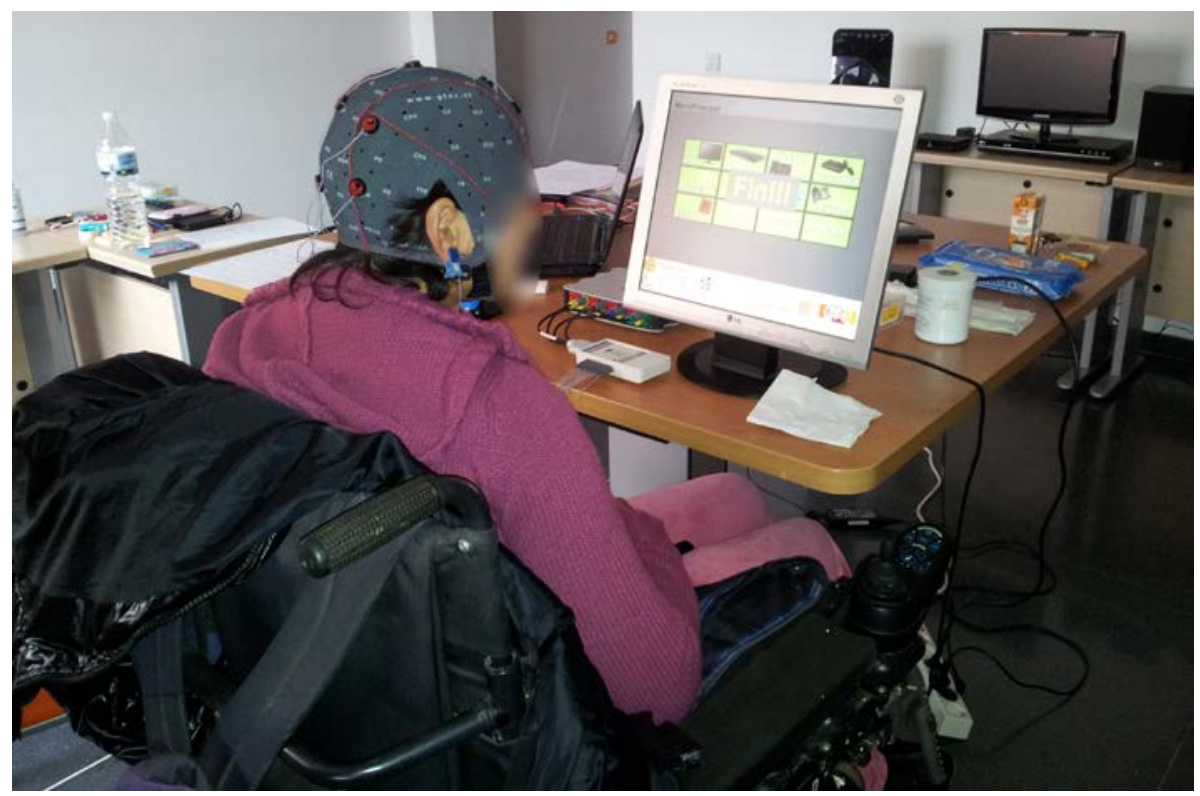

Figure 4-10. Picture of a participant during one of the experimental sessions with the BCI tool.

stimuli were presented for each single item selection. Runs were separated by rest intervals of $60 \mathrm{~s}$. Feedback was not provided to participants during Calibration session. Similarly to the procedure applied by Mak et al. (2012), data from the 5 first runs of this session was used to derive the classifier weights for each participant. The obtained classifier was used during the subsequent 5 runs of Calibration session.

\subsubsection{Evaluation 1 session}

Eval1 started with 2 runs comprised of 6 items in copy-spelling mode, using the TV menu matrix, in order to ensure proper performance (Nijboer et al. 2008). Subsequently, subjects interacted with the assistive BCI tool and all the developed submenus. Eval1 session was comprised of at least 7 assessment runs. For each run, users were asked to focus on items across different menus in order to complete a proposed series of at least 6 items, e.g.: “access the TV menu”, "switch the TV on”, "skip to TV channel 5", "return to the main menu”, "access the DVD menu”, "record the actual channel”. 15 sequences of stimuli were presented during this session for each single item selection. The classifier built for each user in the previous Calibration session was applied for the execution of Eval1 and Eval2, unless an improper performance was identified during the initial copy-spelling items of Eval1. In that case, a new classifier was derived by using these initial copy-spelling runs. 


\subsubsection{Evaluation 2 session}

Eval2 session was analogous to Eval1 session describe above. At the beginning, subjects performed 2 runs comprised of 6 items facing the TV menu matrix in copy-spelling mode for the purpose of ensuring proper performance (Nijboer et al. 2008). Then, participants were asked to attend items across different menus in order to complete several previously proposed tasks. In order to reduce the selection time during Eval2, the number of sequences of stimuli was decreased for each user. Recordings from previous sessions were analysed offline providing accuracy as a function of the number of sequences. Subsequently, the minimum number of sequences that allowed to properly identify all targets was assigned for each user to perform Eval2 tasks. The lowest number of sequences assigned was 2, corresponding to the subjects with the highest offline performance (P25 and P29). The less the number of sequences necessary for suitably identifying the P300 response, the faster the participants can navigate through the assistive BCI tool.

\subsubsection{Assessment}

Participants were required to reach minimum accuracy of 70\% either over the last runs of Calibration session or over the initial copy-spelling runs of the evaluation sessions, since this level is regarded as the minimum level of communication accuracy (Kleih et al. 2010, Kübler et al. 2001). Subjects, who did not satisfy this requirement, performed again Calibration tasks during the subsequent session. Moreover, subjects, who did not achieve online accuracies higher than $70 \%$ during Eval1 session, performed again Eval1 tasks during the following session. Figure 4-11 illustrates the assessment stages.

Performance results were measured in terms of online accuracy (ACC) for each session, selection rate $(S R)$ or speed of selection and information transfer rate (ITR). On one hand, online accuracy was measured as the number of hits divided by the total number of steps of all the proposed sequences to the user:

$$
\text { ACC }=\frac{\text { number of hits }}{\text { number of total selections }} \text {. }
$$

A hit is considered when the application correctly identifies the desired command (target). For the Calibration session, online ACC was measured by means of 


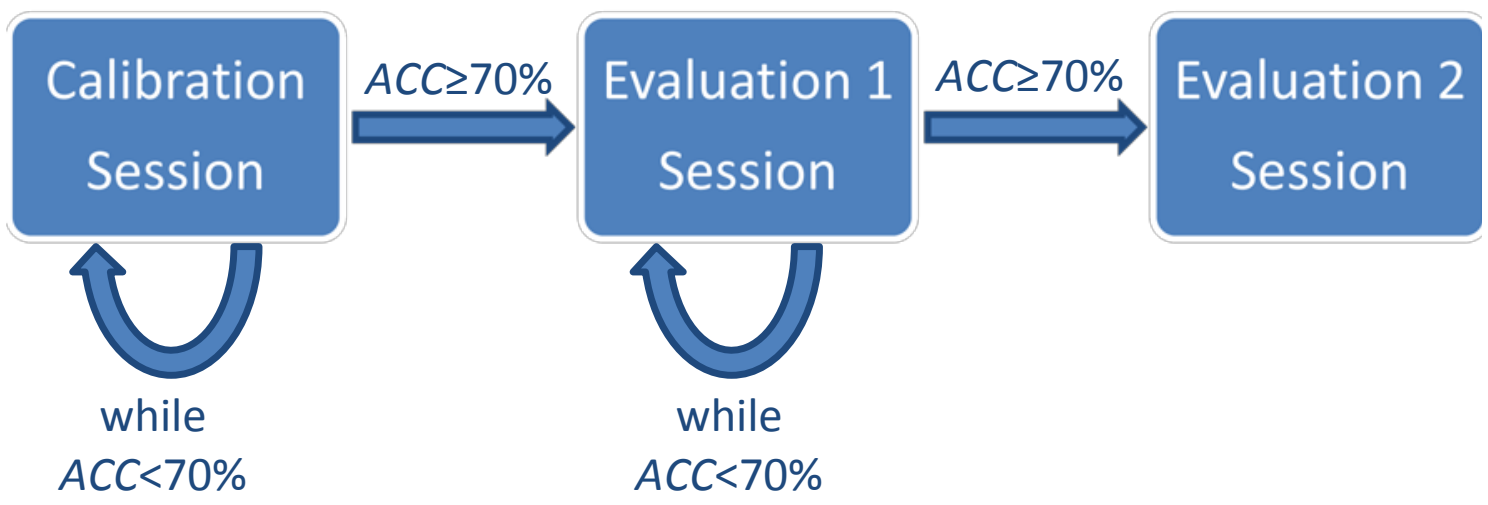

Figure 4-11. Assessment procedure for this study. Each subject was intended to perform three sessions: one session comprised of calibration tasks and two evaluation sessions for assessing their performance with the designed BCI tool (Evaluation1 and Evaluation 2).

the classifier built using the 5 first runs and then applied over the subsequent runs of this session. For the evaluation sessions (Eval1 and Eval2), ACC was measured as the percentage of items properly classified according to a series of proposed tasks. On the other hand, the maximum $S R$ and ITR were also provided, measured in item per minute (item/min) and bit per minute (bit/min), respectively.

$$
\begin{gathered}
S R=\frac{\text { number of total selections }}{\text { time elapsed during these selections }} \text {, and } \\
I T R=\left[\log _{2} N_{S}+A C C \cdot \log _{2} A C C+(1-A P P) \cdot \log _{2}\left(\frac{1-A C C}{N_{S}-1}\right)\right] \cdot S R,
\end{gathered}
$$

where $N_{s}$ is the number of symbols or possible choices presented to the user (Aloise et al. 2011a). Both values are related to the operating speed of the BCI tool regarding the time required to execute a single command, which varies with each user. Hence, the users who achieved higher $S R$ values completed the control sequence commands faster than the users who presented lower $S R$ values. Moreover, the users who achieved higher ITR values completed the control sequence commands faster and with higher accuracy than the users who presented lower ITR values. Since a large interval (5 seconds) was set in order to let users enough time to see the effects of their selections and to focus on the next step of the sequence, ITRs with and without pause between selections were provided in order to compare ITR values with other studies.

Lastly, at the end of the last evaluation session, all participants completed a questionnaire in order to assess the proposed BCI tool. The questionnaire was 
comprised of 11 statements, described in Table 4-2. Participants evaluated different features of the proposed tool such as the paradigm and tasks, the application's design, the degree of difficulty, the usefulness and general aspects about their satisfaction with the experimental phase. They rated each assertion on a 5-point Likert scale (Allison et al. 2010, Münßinger et al. 2010): 0-strongly disagree, 1-disagree, 2-neutral, 3-agree, 4strongly agree. Odd statements were positive assertions related to the assistive BCI tool (i.e. ideal score: 4), whereas even statements were negative assertions (i.e. ideal score: $0)$. 
1. The proposed task (i.e., counting how many times a specific item flickers) is simple

\begin{tabular}{|l|l|l|l|l|}
\hline a. Strongly disagree & b. Disagree & c. Neutral & d. Agree & e. Strongly agree \\
\hline & & & & \\
\hline
\end{tabular}

2. Flickering stimuli are annoying

\begin{tabular}{|l|c|c|c|c|}
\hline a. Strongly disagree & b. Disagree & c. Neutral & d. Agree & e. Strongly agree \\
\hline & & & & \\
\hline
\end{tabular}

3. The assistive BCI tool for operating electronic devices at home is useful to increase the autonomy of disabled people at home

\begin{tabular}{|l|l|l|l|l|}
\hline a. Strongly disagree & b. Disagree & c. Neutral & d. Agree & e. Strongly agree \\
\hline & & & & \\
\hline
\end{tabular}

4. Pictures of each item do not describe suitably the functionality they depict

\begin{tabular}{|c|c|c|c|c|}
\hline a. Strongly disagree & b. Disagree & c. Neutral & d. Agree & e. Strongly agree \\
\hline & & & & \\
\hline
\end{tabular}

5. The assistive BCI tool would be useful to control other devices at home: blinds, doors, bell...

\begin{tabular}{|l|l|l|l|l|}
\hline a. Strongly disagree & b. Disagree & c. Neutral & d. Agree & e. Strongly agree \\
\hline & & & & \\
\hline
\end{tabular}

6. Brain activity acquisition by means of EEG with a cap and active electrodes is not very practical on a daily basis

\begin{tabular}{|l|c|c|c|c|}
\hline a. Strongly disagree & b. Disagree & c. Neutral & d. Agree & e. Strongly agree \\
\hline & & & & \\
\hline 7.
\end{tabular}

7. The assistive BCI tool would be useful out of home for mobility applications

\begin{tabular}{|l|c|c|c|c|}
\hline a. Strongly disagree & b. Disagree & c. Neutral & d. Agree & e. Strongly agree \\
\hline & & & & \\
\hline
\end{tabular}

8. Navigating through different menus is unnecessarily complex

\begin{tabular}{|l|l|l|l|l|}
\hline a. Strongly disagree & b. Disagree & c. Neutral & d. Agree & e. Strongly agree \\
\hline & & & & \\
\hline
\end{tabular}

9. The length of each session was suitable

\begin{tabular}{|l|c|c|c|c|}
\hline a. Strongly disagree & b. Disagree & c. Neutral & d. Agree & e. Strongly agree \\
\hline & \multicolumn{5}{|l|}{} \\
\hline 10.I would not use this assistive BCI tool again \\
\hline a. Strongly disagree & b. Disagree & c. Neutral & d. Agree & e. Strongly agree \\
\hline & \multicolumn{1}{|l}{} \\
\hline \begin{tabular}{l} 
11.I would take part in a similar study again \\
\hline a. Strongly disagree
\end{tabular} b. Disagree & c. Neutral & d. Agree & e. Strongly agree \\
\hline & & & & \\
\hline
\end{tabular}

Table 4-2. Assessment questionnaire of the proposed assistive BCI tool (translated from Spanish). All participants completed the questionnaire after having finished all experimental sessions. 


\section{Results}

5.1. Performance of end users managing the assistive tool

5.2. Assessment of the application by means of the end-users 



\subsection{Performance of end users managing the assistive tool}

The proposed assistive BCI tool was assessed by 30 potential end-users from the NRC-D\&D. Regarding the results, Table 5-1 summarizes the performance reached by users during the assessment sessions. Moreover, online ACC achieved per session by each user are graphically illustrated in Figures 5-1 and 5-2.

In regard to the results of Calibration session, twenty five out of the thirty subjects achieved online ACC above 70\% during their first calibration session. Eighteen out of them even reached accuracies higher than 90\%. The remaining users (P6, P11, P13, P15 and P26) were required to repeat Calibration tasks during the subsequent session in order to obtain a suitable classifier. Participant P11 repeated Calibration session twice, since she did not understand properly the task during the first session. Nevertheless, she was able to achieve $80 \%$ accuracy in the next Calibration session. Subject P13 was not able to interact with the proposed BCI tool: it was not possible to derive a reliable classifier for this participant after performing Calibrations tasks three times. Figure 5-3 illustrates the differences in the P300 response waveforms for participant P2, who only performed Calibration tasks once; subject P26, who carried out Calibration tasks twice; and user P13, who performed Calibration tasks during three sessions.

Regarding the results of both evaluation sessions, twenty three out of the twenty nine subjects that performed evaluations tasks were able to control the assistive BCI tool with accuracies higher than $80 \%$. Nineteen out of them even achieved accuracies above 95\%. The remaining participants (P5, P6, P7, P11, P15 and P26) achieved accuracy lower than 70\% during their first Eval1 session. Five out of them repeated Eval1 tasks during the following session and all of them, except P5 and P26, improved their accuracy results. P7 even reached 77\% accuracy in the repeated Eval1 session.

Finally, in relation to $S R$ and $I T R$, participants who reached high accuracies during the evaluation sessions could operate the assistive tool with fewer number of stimuli sequences and thus faster. SR ranged from 2.23 up to $6.82 \mathrm{item} / \mathrm{min}$ and ITR varied from 2.19 up to $25.91 \mathrm{bit} / \mathrm{min}$ if the pause between selections was taken into account; whereas $S R$ ranged from 2.87 up to 21.49 item/min and ITR varied from 2.82 up to $81.58 \mathrm{bit} / \mathrm{min}$ without the pause between selections. In fact, the subjects that 


\begin{tabular}{|c|c|c|c|c|c|}
\hline Subject & $\begin{array}{c}\text { Calibration session } \\
\text { ACC (\%) }\end{array}$ & $\begin{array}{c}\text { Eval1 session } \\
\text { ACC }(\%)\end{array}$ & $\begin{array}{c}\text { Eval2 session } \\
\text { ACC }(\%)\end{array}$ & $\begin{array}{c}\text { Max } S R \\
\text { (item/min) }\end{array}$ & $\begin{array}{l}\text { Max ITR } \\
\text { (bit/min) }\end{array}$ \\
\hline $\mathrm{P} 1$ & 100.00 & 96.55 & 95.65 & $4.17(7.16)$ & $14.10(24.20)$ \\
\hline $\mathrm{P} 2$ & 100.00 & 100.00 & 91.04 & 5.89 (14.33) & $17.85(43.42)$ \\
\hline P3 & 100.00 & 97.06 & 74.51 & $2.64(3.58)$ & $5.37(7.30)$ \\
\hline P4 & 100.00 & 95.00 & 96.00 & $5.89(14.33)$ & $20.06(48.80)$ \\
\hline $\mathrm{P} 5^{*}$ & 71.43 & $55.56 / 46.15$ & - & $2.23(2.87)$ & $2.19(2.82)$ \\
\hline $\mathrm{P}^{*}$ & 68.86 & $46.51 / 64.71$ & - & $2.23(2.87)$ & $2.60(3.35)$ \\
\hline $\mathrm{P}^{*}$ & 78.57 & $46.67 / 77.78$ & - & $2.23(2.87)$ & $3.22(4.14)$ \\
\hline P8 & 91.67 & 100.00 & 92.86 & $4.17(7.16)$ & $13.20(22.65)$ \\
\hline P9 & 96.67 & 100.00 & 90.48 & $4.17(7.16)$ & $12.48(21.43)$ \\
\hline P10 & 82.35 & 75.76 & 84.38 & $2.64(3.58)$ & $6.84(9.29)$ \\
\hline $\mathrm{P} 11^{* *}$ & 38.89 / 80.00 & 63.33 & - & $2.23(2.87)$ & $3.33(4.29)$ \\
\hline $\mathrm{P} 12$ & 87.50 & 100.00 & 84.62 & $5.89(14.33)$ & 15.37 (37.39) \\
\hline $\mathrm{P} 13^{* * *}$ & 27.78 / 33.33 / 37.50 & - & - & - & - \\
\hline P14 & 95.83 & 100.00 & 78.46 & $4.17(7.16)$ & 9.39 (16.12) \\
\hline $\mathrm{P} 15^{*}$ & 67.86 & $60.00 / 65.00$ & - & $2.23(2.87)$ & $3.25(4.18)$ \\
\hline P16 & 100.00 & 95.24 & 71.83 & $3.23(4.78)$ & $6.14(9.07)$ \\
\hline P17 & 100.00 & 100.00 & 93.55 & 2.81 (3.91) & $9.03(12.56)$ \\
\hline P18 & 90.91 & 89.39 & 96.72 & $4.62(8.60)$ & $16.03(29.80)$ \\
\hline P19 & 86.36 & 95.00 & 68.52 & 2.48 (3.31) & $4.31(5.74)$ \\
\hline P20 & 100.00 & 93.55 & 82.61 & $2.81(3.91)$ & $6.99(9.72)$ \\
\hline P21 & 86.36 & 96.77 & 93.55 & 2.81 (3.91) & $9.03(12.56)$ \\
\hline P22 & 100.00 & 96.83 & 94.20 & $4.62(8.60)$ & $15.08(28.05)$ \\
\hline P23 & 95.45 & 80.65 & 96.67 & $3.50(5.37)$ & $12.10(18.60)$ \\
\hline P24 & 100.00 & 100.00 & 96.72 & 5.89 (14.33) & 20.42 (49.67) \\
\hline P25 & 100.00 & 95.24 & 100.00 & $6.82(21.49)$ & 25.91 (81.58) \\
\hline P26 ${ }^{*}$ & 66.67 & 38.16 / 36.84 & - & - & - \\
\hline P27 & 100.00 & 82.54 & 94.55 & 3.50 (5.37) & 11.50 (17.68) \\
\hline P28 & 90.91 & 88.24 & 87.69 & $3.01(4.30)$ & $8.43(12.05)$ \\
\hline P29 & 100.00 & 96.77 & 97.14 & $6.82(21.49)$ & 23.91 (75.29) \\
\hline P30 & 77.27 & 96.77 & 89.06 & $2.81(3.91)$ & $8.13(11.31)$ \\
\hline
\end{tabular}

Table 5-1. Results for each participant managing the assistive BCI tool. Online accuracy (ACC) for each kind of session, maximum item selection rate (SR) with the pause between selections (and without the pause between selections in parentheses), and maximum information transfer rate (ITR) with the pause between selections (and without the pause between selections in parentheses).

*: During the third session these participants repeated Eval1 tasks, due to accuracy lower than $70 \%$ during the previous Eval1 session.

**: During the second session this participant repeated Calibration tasks, due to accuracy lower than $70 \%$ during the previous Calibration session. During the third session, this participant performed Eval1 tasks.

${ }^{* * *}$ : During the second and third sessions this participant repeated Calibration tasks , due to accuracy lower than $70 \%$ during the previous Calibration sessions. 


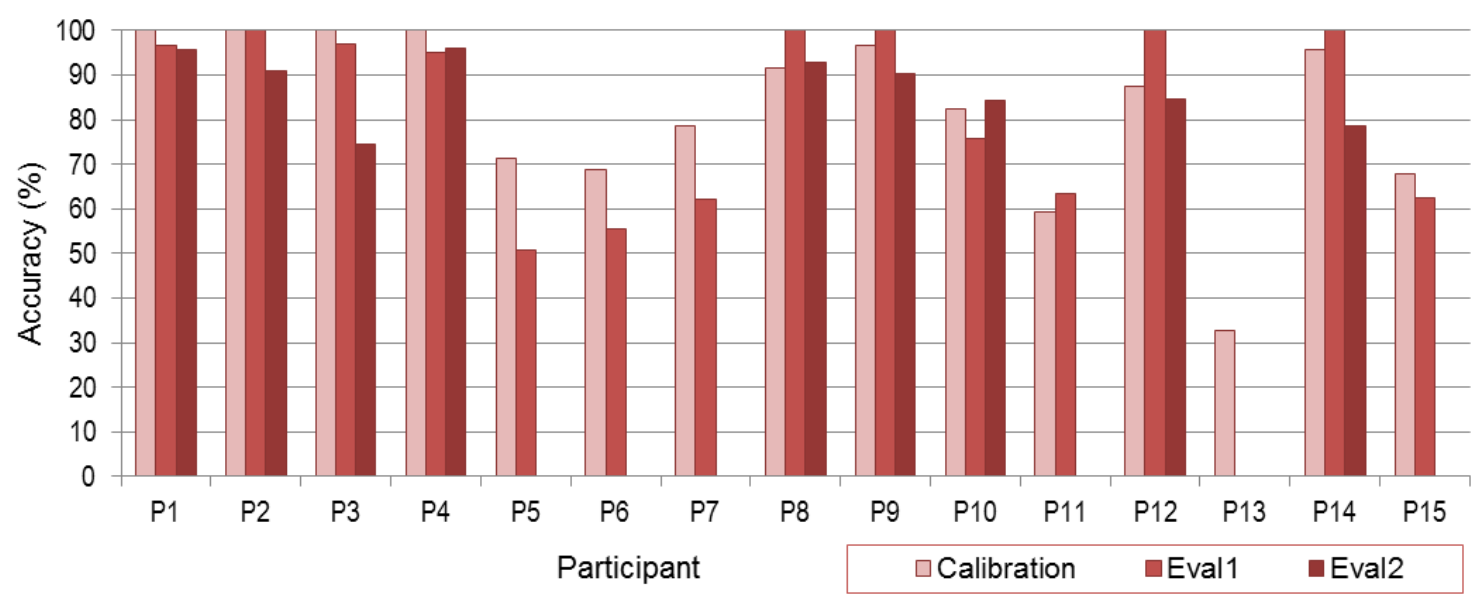

Figure 5-1. Results of the first group of participants managing the assistive BCI tool. Online accuracies (ACC) for Calibration, Eval1 and Eval2 sessions are shown.

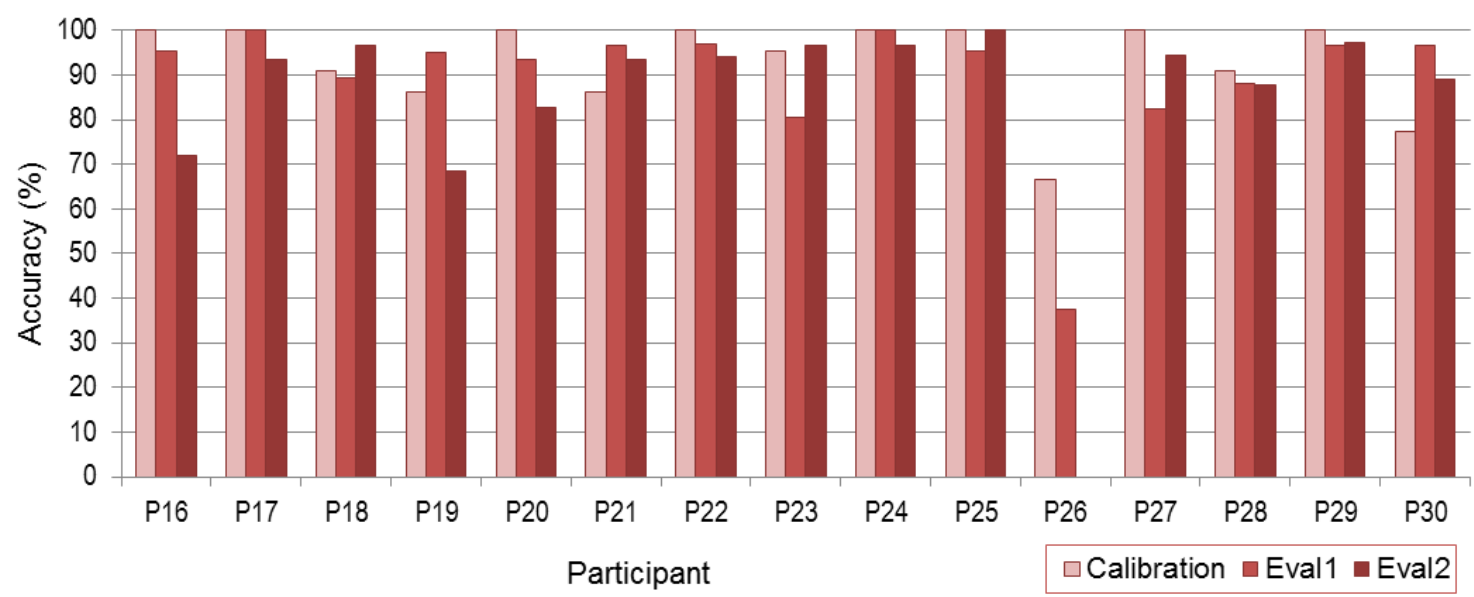

Figure 5-2. Results of the second group of participants managing the assistive BCI tool. Online accuracies (ACC) for Calibration, Eval1 and Eval2 sessions are shown.

achieved the highest performance results (P4, P24, P25 and P29) reached ITR values above $20 \mathrm{bit} / \mathrm{min}$ managing the proposed tool.

\subsection{Assessment of the application by means of the end-users}

Thirty severely impaired peopled evaluated the assistive BCI tool. Figure 54 shows two participants managing different electronic devices with the proposed tool during the experimental stage. All subjects completed an assessment questionnaire immediately after finishing the last evaluation session. Each user had to rate 11 
(a)

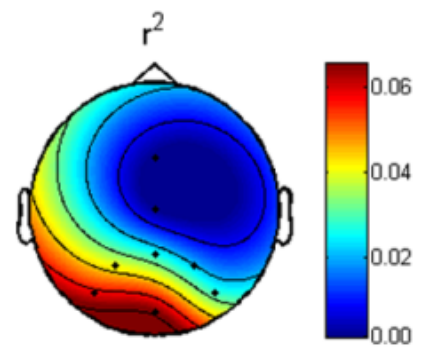

(b)

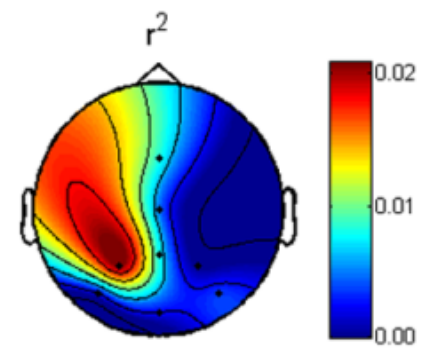

(c)

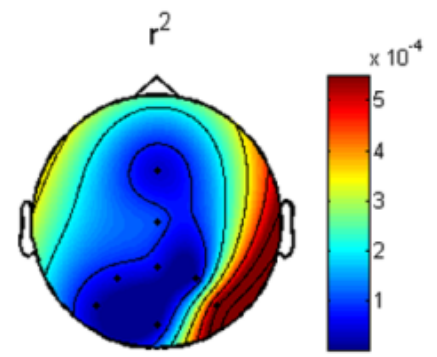

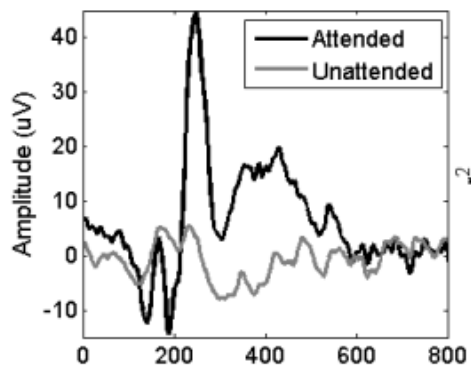
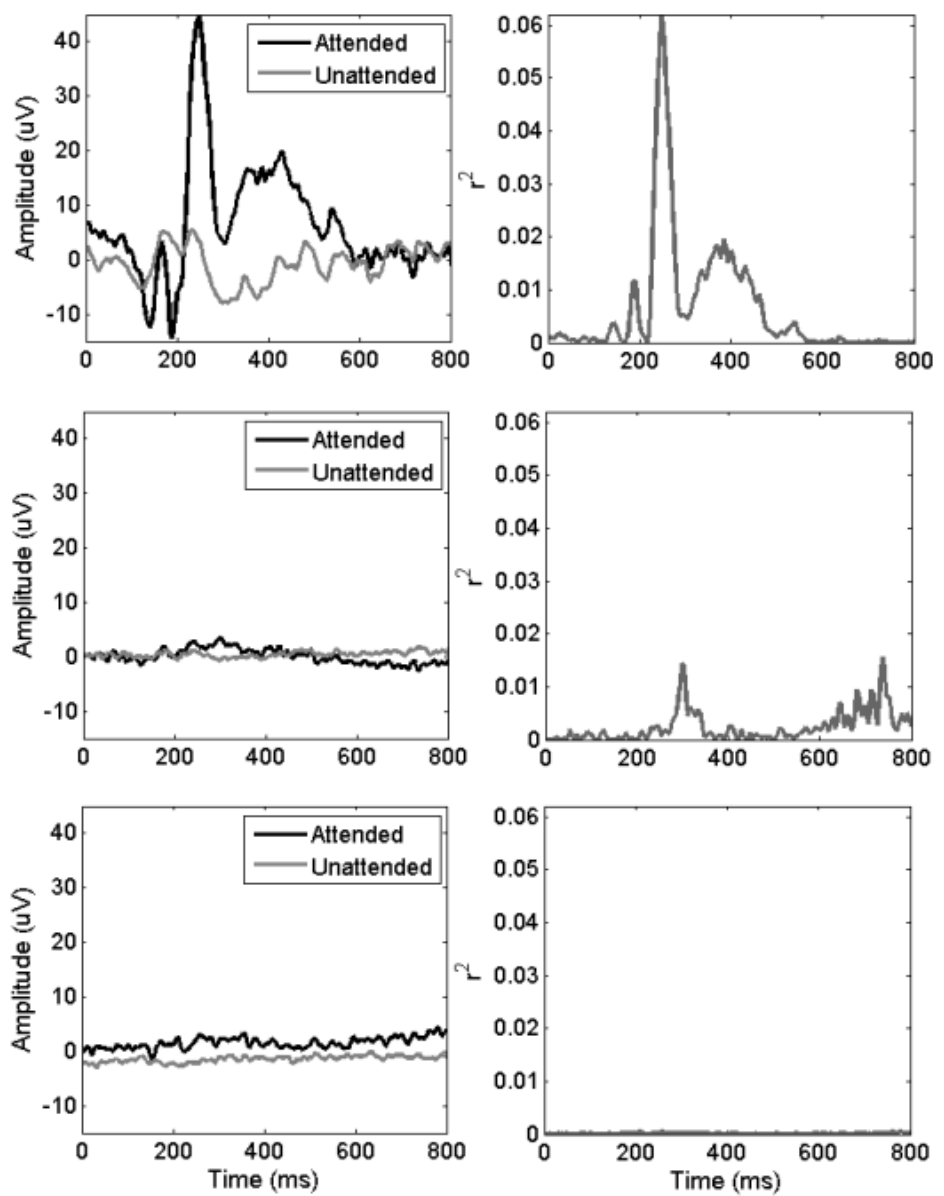

Figure 5-3. Topographical distribution of P300 response (first column), averaged response according to the stimulus type (second column), and $\boldsymbol{r}^{2}$ waveform (third column) for: (a) participant P2, who only performed Calibration tasks once; (b) subject P26, who carried out Calibration tasks twice; (c) and user P13, who performed Calibration tasks during three sessions. Usually, the more prominent the P300 amplitude peak is, the more accuracy is reached by the user.

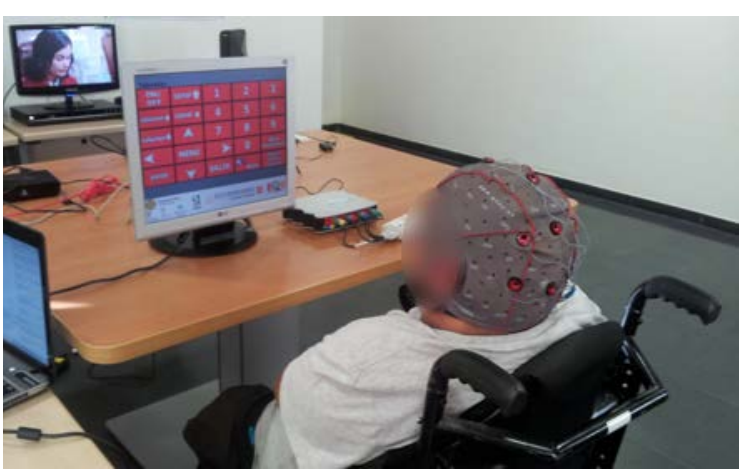

(a)

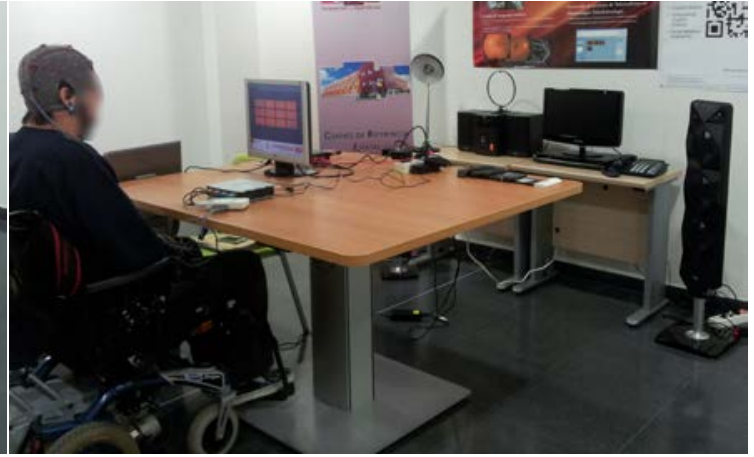

(b)

Figure 5-4. Pictures of two participants using the assistive BCI tool to manage different electronic devices: (a) TV, (b) Fan. 
statements on a 5-point Likert scale. Subjects evaluated the design, interface, usability and usefulness of the BCI tool. The distribution of the responses for each statement is described below. Moreover, Figure 5-5 shows graphically the distribution of responses to the questionnaire.

1. The proposed task (i.e., counting how many times a specific item flickers) is simple
a. Strongly disagree
$0.00 \%$
b. Disagree
$0.00 \%$
c. Neutral
$30.00 \%$
d. Agree
$20.00 \%$
e. Totally agree
$50.00 \%$

\section{Flickering stimuli are annoying}
a. Strongly disagree
$\mathbf{4 0 . 0 0 \%}$
b. Disagree
$13.33 \%$
c. Neutral
$20.00 \%$
d. Agree
$23.33 \%$
e. Totally agree
$3.33 \%$

3. The assistive BCI tool for operating electronic devices at home is useful to increase the autonomy of disabled people at home
a. Strongly disagree
$0.00 \%$
b. Disagree
$6.67 \%$
c. Neutral
$0.00 \%$
d. Agree
$43.33 \%$
e. Totally agree
$50.00 \%$

4. Pictures of each item do not describe suitably the functionality they depict
a. Strongly disagree
$73.33 \%$
b. Disagree
$16.67 \%$
c. Neutral
$6.67 \%$
d. Agree
$0.00 \%$
e. Totally agree
$3.33 \%$

5. The assistive BCI tool would be useful to control other devices at home: blinds, doors, bell...
a. Strongly disagree
$0.00 \%$
b. Disagree
$6.67 \%$
c. Neutral
$0.00 \%$
d. Agree
$30.33 \%$
e. Totally agree
$63.33 \%$ 
6. Brain activity acquisition by means of EEG with a cap and active electrodes is not very practical on a daily basis

a. Strongly disagree $10.00 \%$

$\begin{array}{ll}\text { b. Disagree } & 16.67 \%\end{array}$

$\begin{array}{ll}\text { c. Neutral } & 23.33 \%\end{array}$

$\begin{array}{ll}\text { d. Agree } & \mathbf{3 6 . 6 7 \%}\end{array}$

$\begin{array}{ll}\text { e. Totally agree } & 13.33 \%\end{array}$

7. The assistive BCI tool would be useful out of home for mobility applications
a. Strongly disagree
$0.00 \%$
b. Disagree
$3.33 \%$
c. Neutral
$46.67 \%$
d. Agree
$26.67 \%$
e. Totally agree
$23.33 \%$

8. Navigating through different menus is unnecessarily complex
a. Strongly disagree
$30.00 \%$
b. Disagree
46.67\%
c. Neutral
$13.33 \%$
d. Agree
$6.67 \%$
e. Totally agree
$3.33 \%$

9. The length of each session was suitable
a. Strongly disagree
$0.00 \%$
b. Disagree
$10.00 \%$
c. Neutral
$6.67 \%$
d. Agree
$40.00 \%$
e. Totally agree
$43.33 \%$

10.I would not use this assistive BCI tool again
a. Strongly disagree
$53.33 \%$
b. Disagree
$40.00 \%$
c. Neutral
$3.33 \%$
d. Agree
$3.33 \%$
e. Totally agree
$0.00 \%$

11.I would take part in a similar study again
a. Strongly disagree
b. Disagree
c. Neutral
d. Agree
$0.00 \%$
e. Totally agree
$\mathbf{9 3 . 3 3 \%}$ 


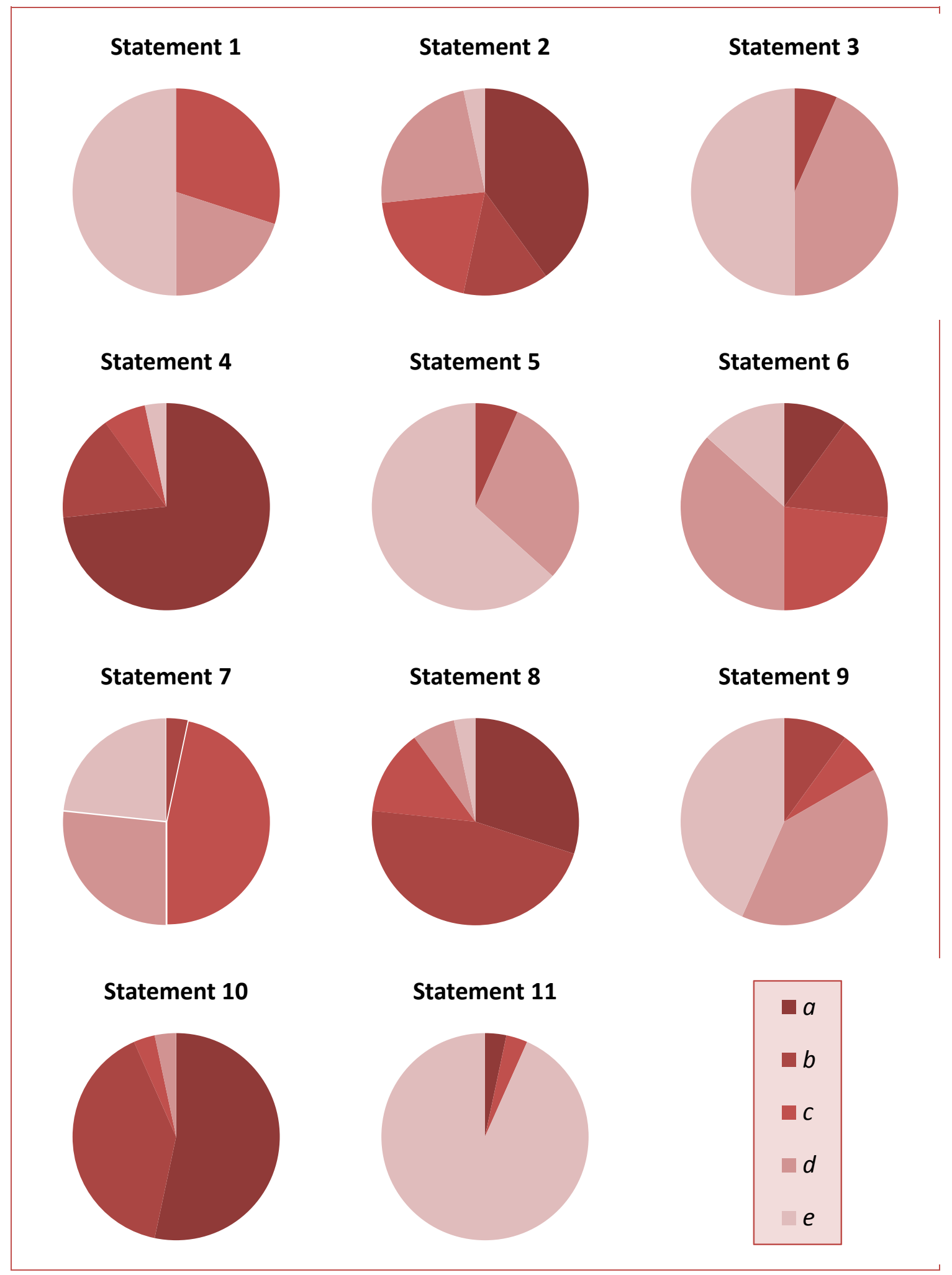

Figure 5-5. Graphical distribution of responses given by the participants to each statement of the assessment questionnaire. Odd statements were positive assertions about the proposed BCI tool (ideal response: $e$ ), whereas even statements were negative assertions (ideal response: $a$ ). 
Table 5-2 and Figure 5-6 illustrates the averages and standard deviations of the scores assigned by the participants to each statement of the questionnaire. All odd statements were rated by participants with average scores higher than 2 (ideal score: 4), whereas four out of the five even negative statements were evaluated with average scores lower than 2 (ideal score: 0 ). Therefore, the distribution of the responses and the average scores show that most of the participants assessed favourably the proposed assistive BCI tool. Only the statement 6, about the signal acquisition on a daily basis by means of EEG with a standard cap, was rated unfavourably.

\begin{tabular}{llcc}
\hline \multirow{2}{*}{ Statement } & \multicolumn{2}{c}{ Score } \\
\cline { 3 - 4 } & Mean & SD \\
\hline 1 & $\begin{array}{l}\text { The proposed task (i.e., counting how many times a specific item flickers) is } \\
\text { simple }\end{array}$ & 3.20 & 0.89 \\
2 & Flickering stimuli are annoying & 1.37 & 1.33 \\
3 & $\begin{array}{l}\text { The assistive BCI tool for operating electronic devices at home is useful to } \\
\text { increase the autonomy of disabled people at home }\end{array}$ & 3.37 & 0.81 \\
4 & Pictures of each item do not describe suitably the functionality they depict & 0.43 & 0.90 \\
5 & $\begin{array}{l}\text { The assistive BCI tool would be useful to control other devices at home: } \\
\text { blinds, doors, bell... }\end{array}$ & 3.50 & 0.82 \\
6 & Brain activity acquisition by means of EEG with a cap and active electrodes & 2.27 & 1.20 \\
7 & is not very practical on a daily basis & & \\
8 & Nhe assistive BCI tool would be useful out of home for mobility applications & 2.70 & 0.88 \\
9 & The length of each session was suitable & 1.07 & 1.01 \\
10 & I would not use this assistive BCI tool again & 3.17 & 0.95 \\
11 & I would take part in a similar study again & 0.57 & 0.73 \\
\hline
\end{tabular}

Table 5-2. Questionnaire results for the assessment of the assistive BCI tool.

Each statement was rated on a 5-point Likert scale (0-strongly disagree, 1-disagree, 2-neutral, 3agree, 4-strongly agree). Odd statements were positive assertions about the assistive BCI tool (ideal score: 4) while even statements were negative assertions (ideal score: 0 ). 


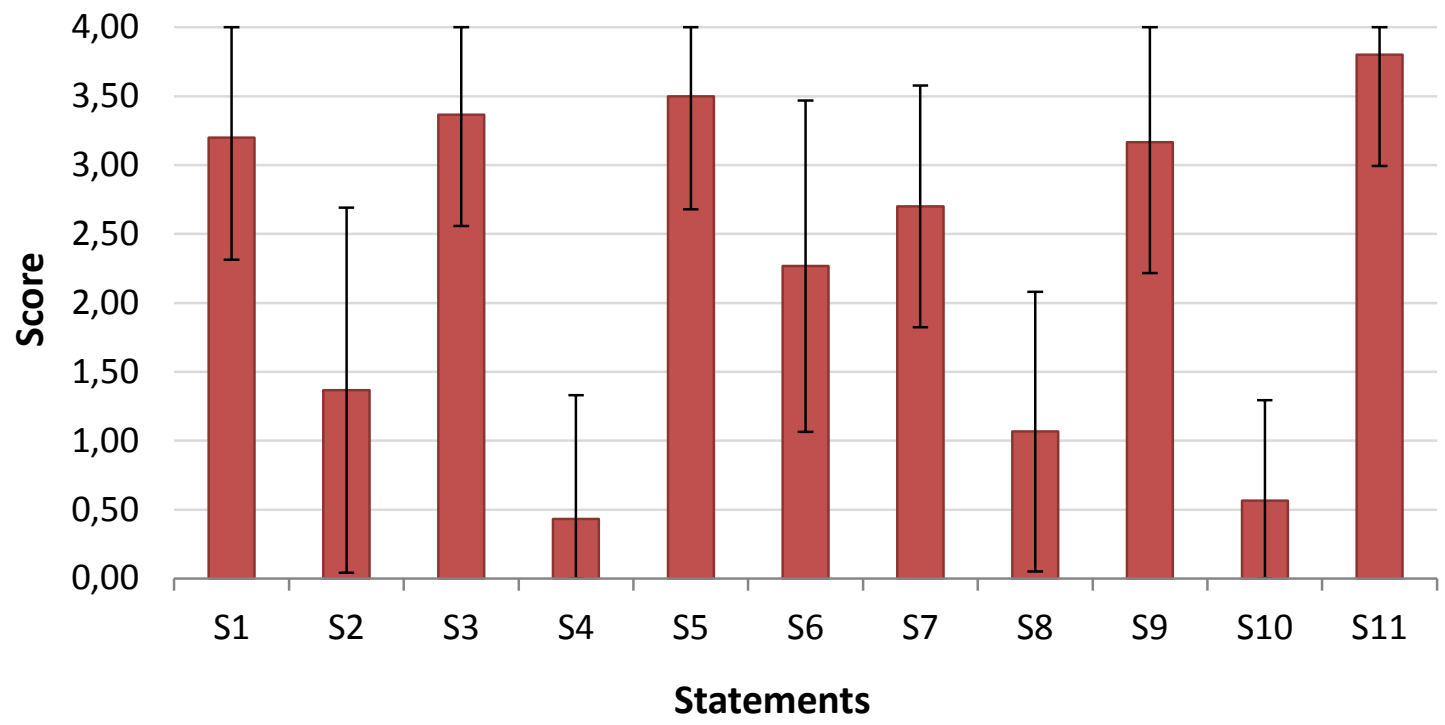

Figure 5-6. Averages and standard deviations of the scores assigned by the participants to each statement of the questionnaire. Odd statements were positive assertions about the proposed BCI tool (ideal score: 4), whereas even statements were negative assertions (ideal score: 0 ). 



\section{Discussion}

\subsection{Introduction}

6.2. Cognitive vs motor impairments

6.3. Luria test

6.4. P300-based studies involving potential end-users

6.4.1. Speller

6.4.2. Home automation control

6.5. Previous approaches

6.6. Real end-users assessment

6.7. Limitations 



\subsection{Introduction}

In the present study an assistive P300-based BCI tool was researched in order to assess its usefulness at home for people with severe impairments. Thirty severely disabled users interacted with the BCI tool for assessing its performance and usefulness for their daily life. Twenty three out of the thirty participants were able to properly manage the assistive BCI tool, with accuracy above $80 \%$. Nineteen out of them reached accuracies higher than 95\%. These results are noteworthy since the participants were real end-users of BCIs because of motor disabilities. Three subjects (P6, P11 and P15) managed the proposed tool during the assessment session with accuracy ranging from $60 \%$ to 65\%; whereas the remaining three users (P5, P13 and P26) could not properly operate the assistive BCI tool. In the particular case of subject P13, he was not able to perform assessment sessions with the proposed application. After three repetitions of the Calibration session, it was not possible to identify reliable changes in his EEG between the desired target and all other items. This could be due to subject P13 presented numerous sudden muscle spasms affecting the quality of EEG recordings.

\subsection{Cognitive versus motor impairments}

In regard to motor impairment, twenty four subjects in the study presented severe or moderate motor disabilities. Despite their high degree of motor impairment, sixteen out of them (P2, P3, P4, P8, P9, P14, P16, P18, P19, P20, P21, P22, P25, P27, P29 and P30) reached outstanding accuracy managing the proposed tool, above $90 \%$. The remaining users with severe or moderate motor impairments achieved accuracies higher than 75\% (P7, P10 and P28) or moderate accuracies (P6, P11 and P15). Finally, only two participants with high degree of motor disability (P5 and P13) were not able to suitably operate the assistive BCI tool.

In terms of cognitive impairment, twelve subjects did not have any cognitive disabilities. Eleven out of them (P1, P17, P18, P19, P20, P22, P25, P27, P28, P29 and P30) achieved accuracies above 88\% managing the BCI tool, whereas P6 reached 64\% accuracy after having performed Eval1 session twice. In the particular case of subject P6, he appeared to be barely motivated during the experimental sessions. Carrillo de la Peña and Cadaveira (2000) showed that the P300 response is affected by 
non-specific factors, such as the motivation to perform a task properly. This could be the reason for the poor performance of participant P6 and his unsteady results across sessions. Regarding the eleven participants who presented mild cognitive impairments, eight out of them (P2, P3, P4, P8, P9, P12, P16 and P23) achieved accuracy higher than 95\%; one out of them (P1) reached accuracy of 77\%; whereas two out of them (P13 and P26) were not able to suitably operated the proposed tool. Lastly, attending to the seven participants with the highest degree of cognitive impairment, four out of them (P10, P14, P21 and P24) reached accuracies higher than 84\%; two out of them (P11 and P15) achieved accuracies ranging from $63 \%$ to $65 \%$; and one out of them (P5) was not able to properly manage the BCI tool.

Concerning the sustained attention ability, participants P11, P24 and P26 presented the worst assessment, poor or very poor ability. P11 had to repeat Calibration tasks twice since she did not properly understand the task the first time. Then, she achieved 80\% accuracy in Calibration tasks but only 63\% during the Eval1 assessment session. P24 achieved accuracy of 100\% managing the proposed BCI tool, despite her poor sustained attention ability, and, finally, P26 who was the participant with the worst attention ability, was not able to suitably operate the assistive tool.

Respect to the most severe participants (P5, P10, P11, P14, P15 and P21), with both severe/moderate motor disability and moderate cognitive impairments, five out of them reached results ranging from modest (63\%) to excellent (100\%) during at least one of the assessment sessions. Only P5 was not able to properly manage the assistive BCI tool. These results are encouraging since most of the population of the study presented not only motor impairment but also cognitive disabilities. Piccione et al. (2006) proved that severely disabled subjects could use a P300-based BCI although their performance was influenced by the degree of impairment. In contrast, Nijboer et al. (2008) showed that the correlation between the degree of disability and the online performance with the BCI system did not reach statistical significance. The latter study agree with the present results, since statistical significance was not achieved in the correlation between the online BCI performance and the degree of motor impairment ( $r=0.1961, p=0.2991)$, cognitive impairment $(r=0.2229, p=0.2364)$ or sustained attention ability $(r=0.3337, p=0.0715)$ of the thirty participants. 


\subsection{Luria test}

The participants recruited at the second stage, P16 to P30, performed the Luria AND test (Christensen 1987). It included nine specific sub-tests for assessing nine functions distributed among five different areas: visuospatial (visual perception and spatial orientation), oral language (receptive speech and expressive speech), memory (immediate memory and logical memorization), intelligence (thematic draws and conceptual activity) and attention (attentional control). The correlation between the online BCI performance and the scores of Luria sub-tests for these participants was analysed. Statistical significance $(r=7986, p=0.0004)$ was found only for the correlation between the online accuracy in the evaluation sessions and the attentional control function's score. This analysis suggests that the performance could be influenced by the users' attentional control function. It also concords with Piccione et al. (2006), since this function usually depends on each specific pathology and its stage. Nevertheless, Luria scores were only available for the second group of patients, composed of 15 individuals; whereas the sustained attention ability degree, which was provided for both groups of patients (30 subjects), did not show significant relation with the users' performance. Therefore, the attention ability of patients would probably be the only requirement for using a P300-based BCI, independently of the specific pathology and the degree of impairment.

\subsection{P300-based studies involving potential end-users}

The studies related to BCIs typically only involve healthy participants. However, severely impaired people are the real end-users of these systems because their personal autonomy can be really improved. Table 6-1 compares seven studies that assessed P300-based BCIs with severely disabled subjects. The last row summarizes the present study.

\subsubsection{Speller}

Firstly, Sellers and Donchin (2006) found that both healthy subjects and subjects with advanced ALS could use a P300-based BCI using auditory or visual 
stimuli. Second, Nijboer et al. (2008) extended these studies involving severely paralysed people caused by ALS. They were able to use a P300-based BCI that employed a variable-sized matrix of characters for cued and spontaneous text production. Four out of the participants completed the experiment. Their mean accuracy ranged from $46 \%$ to $92 \%$. Moreover, Nijboer et al. (2008) performed an exhaustive study over 40 weeks and showed that the amplitude and latency of the P300 potential remained steady over this period of time. Recently, Ortner et al. (2011) analysed the BCI performance of a young healthy population and a group of 15 people with motor disability. They observed that their impairments affected the performance of the latter group. Therefore, the development of automatic algorithms for removing artefacts is required. Furthermore, properly adjust the parameters of the environment and the paradigm for each patient is essential. Mak et al. (2012) identified EEG features that correlate with P300-based BCI performance assessed on twenty patients with advanced ALS. Eleven out of the twenty participants reached accuracy of $100 \%$ during at least one copy-spelling run using a 6 x 6 speller matrix. Finally, the study of Spüler et al. (2012) included processing of error-related potentials in order to identify when the classifier did not select the user's desired result. Error-related potentials information allowed to increase accuracy performance in both healthy and severely impaired people. A population of six severely disabled subjects reached a mean accuracy of $74 \%$ applying this methodology.

\subsubsection{Home automation control}

In regard to previous studies related to BCI applications for home automation control, Hoffman et al. (2008) evaluated a P300-based BCI with five disabled subjects due to different pathologies and four healthy subjects simulating an environment control paradigm. Four out of the five disabled participants achieved 100\% offline accuracy, as well as two out of the healthy subjects. Nevertheless, in terms of ITR, healthy subjects achieved higher maximum bitrates than impaired subjects. However, this study was performed using a quite different P300 paradigm. One matrix, consisted of six images that flashed one by one, was used as stimuli matrix. Furthermore, only four sessions comprised of 6 single item selections each were carried out. In the present study, the BCI application comprises 137 items from 10 different menus or matrices, stimuli are presented over rows and columns (RC pattern approach) 


\begin{tabular}{|c|c|c|c|c|c|c|c|}
\hline Author & Subjects & $\begin{array}{l}\text { Paradigm } \\
\text { description }\end{array}$ & Application & $\begin{array}{l}\text { Sessions / } \\
\text { Total item } \\
\text { selections }\end{array}$ & Sequences & $\begin{array}{c}\text { Mean } \\
\text { ACC (\%) }\end{array}$ & $\begin{array}{c}I T R \\
\text { (bit/min) }\end{array}$ \\
\hline $\begin{array}{l}\text { Sellers and } \\
\text { Donchin } \\
\text { (2006) }\end{array}$ & 3 & $\begin{array}{l}4 \text { separately words } \\
\text { Visual and/or } \\
\text { auditive stimuli }\end{array}$ & $\begin{array}{l}\text { 4-choice } \\
\text { communicator } \\
\text { (YES, NO, } \\
\text { PASS, END) }\end{array}$ & $10 / 120$ & 25 & 64.8 & 0.7 \\
\hline $\begin{array}{l}\text { Nijboer et } \\
\text { al. (2008) }\end{array}$ & $4^{*}$ & $\begin{array}{l}1 \text { matrix } \\
6 x 6 \text { or } 7 x 7 \\
\text { Characters } \\
\text { Row/column }\end{array}$ & Speller & 20 / 660 & $4-20$ & 71.9 & 8.4 \\
\hline $\begin{array}{l}\text { Ortner et } \\
\text { al. (2011) }\end{array}$ & 15 & $\begin{array}{l}1 \text { matrix } \\
10 x 5 \text { or } 6 x 6 \\
\text { Characters } \\
\text { Row/Column }\end{array}$ & Speller & $1 / 10$ & 15 & 70.7 & - \\
\hline $\begin{array}{l}\text { Mak et al. } \\
\text { (2012) }\end{array}$ & 20 & $\begin{array}{l}1 \text { matrix 6x6 } \\
\text { Characters } \\
\text { Checkerboard }\end{array}$ & Speller & $1 / 35$ & - & 58.0 & - \\
\hline $\begin{array}{l}\text { Spüler et } \\
\text { al. (2012) }\end{array}$ & 6 & $\begin{array}{l}1 \text { matrix 6x6 } \\
\text { Characters } \\
\text { Row/column }\end{array}$ & $\begin{array}{l}\text { Speller with } \\
\text { error potentials } \\
\text { detection }\end{array}$ & $2 / 100$ & $2-8$ & 74.0 & 2.1 \\
\hline $\begin{array}{l}\text { Hoffman et } \\
\text { al. (2008) }\end{array}$ & $4^{* *}$ & $\begin{array}{l}1 \text { matrix } 3 \times 2 \\
\text { Images } \\
\text { Individual flashes }\end{array}$ & $\begin{array}{l}\text { Environment } \\
\text { control at home } \\
\text { - Simulated }\end{array}$ & $2 / 24$ & $20-25$ & $100.0^{* * * *}$ & $15.9^{* * *}$ \\
\hline $\begin{array}{l}\text { Aloise et } \\
\text { al. (2011b) }\end{array}$ & 7 & $\begin{array}{l}1 \text { matrix } \\
4 \times 4 \\
\text { Images } \\
\text { Row/Column }\end{array}$ & $\begin{array}{l}\text { Environment } \\
\text { control at home } \\
\text { - Virtual } \\
\text { Asynchronus } \\
\text { operation }\end{array}$ & $2 / 75$ & $1-12$ & 72.7 & - \\
\hline $\begin{array}{l}\text { The } \\
\text { present } \\
\text { study }\end{array}$ & 30 & $\begin{array}{l}10 \text { matrices } \\
3 \times 4,4 \times 4 \text { and } 5 \times 5 \\
\text { Images } \\
\text { Row/column } \\
\end{array}$ & $\begin{array}{l}\text { Environment } \\
\text { control at } \\
\text { home - Real }\end{array}$ & 3 / 134 & $2-15$ & 80.5 & 20.8 \\
\hline
\end{tabular}

Table 6-1. Comparison of different studies involving P300-based BCIs and severely disabled participants. Number of impaired subjects, main details of each paradigm and application studied, number of performed sessions and number of total item selections (trials) across all sessions, number of sequences of stimuli, mean online accuracy (ACC) and maximum information transfer rate (ITR) without pause between selections are provided.

* Four additional subjects were not included since they did not complete the study or algorithms failed to detect reliably the evoked potentials.

** One additional subject was not included since classification accuracies above chance level could not be obtained.

${ }^{* * *}$ Only offline classification results are provided. Four-fold cross-validation was used to estimate the accuracy. 
and two assessment sessions comprised of at least 42 single item selections were performed. More recently, Aloise et al. (2011b) evaluated an asynchronous P300-based BCI for virtual environmental control with seven potential end-users. On average, they achieved $72.7 \%$ accuracy and $1 \%$ error rate in the asynchronous mode. Therefore, the system revealed strong reliability in avoiding false positives when the subjects were engaged in other tasks. As shown in Table 6-1, the present study involved a larger population than previous studies. Although Sellers and Donchin (2006) and Nijboer et al. (2008) carried out long and thorough studies, our study is also one of the most exhaustive since comprised three experimental sessions, consisted of 134 selections. Moreover, the mean online accuracy and maximum ITR were higher than the values reached in previous studies with impaired populations.

\subsection{Previous approaches}

Our initial approach to BCIs for home automation control was by means of motor imagery (Corralejo et al. 2011). MI-based BCIs depend on the user's ability to control his internal electrophysiological activity in order to move a cursor in the screen (Wolpaw et al. 2000). Due to the nature of MI-based BCIs, the number of devices and functionalities controlled by that application were limited: 4 electronic devices (TV, DVD player, Hi-Fi system and lights) and up to 19 control commands. One aim of the present study was to increase the number of devices and commands that the users could manage. In fact, using a P300-based BCI, the assistive tool proposed in this work allows to suitably operate 8 electronic devices (TV, DVD player, Hi-Fi system, multimedia drive, lights, heater, fan, and phone) and up to 137 commands (115 IR control commands and 22 navigation commands). Furthermore, our initial experiments showed high performance results with healthy users, but they also proved difficulties for disabled people. MI-based BCIs require a high degree of concentration ability and an extensive training period to properly move a cursor in 2D. It was shown that cognitive impairments affected the attention ability and thus the MI-based BCI performance (Corralejo et al. 2011). The present study proposes a P300-based assistive BCI tool, which does not require extensive training, since the brain spontaneously responds to 
external stimuli. Actually, this study shows that potential BCI end-users, i.e., people with severe disabilities, could properly manage this kind of BCIs.

Our first experiments also raised the electrodes issue. The set-up of EEG passive electrodes took too much time and this typically had a negative influence on the end-user's motivation. In the present study this issue was avoided by using EEG active electrodes instead of passive ones. Thus, the set-up time was significantly reduced and we observed that users' motivation was not reduced due to the EEG recording set-up.

In summary, the assistive BCI tool proposed in the present study overcomes the main limitations found in our previous BCI approaches for people with severe impairments.

\subsection{Real end-users assessment}

Regarding the end-users assessment of the proposed BCI tool by means of a questionnaire, most of the participants rated favourably the design and usefulness of the assistive tool. More specifically, users considered that the proposed tool was easy to manage. Both the images depicting specific devices and commands, and the navigation system through menus were generally well accepted and understood. Furthermore, participants considered that the proposed assistive tool was really useful to increase their autonomy at home. They were even interested in including more home devices such as blinds, doors or bells; whereas they were not especially interested in BCIs for mobility applications. Nevertheless, participants rated unfavourably the statement 6, i.e., subjects stated that EEG-based BCIs are not very practical on a daily basis. The main issue is the use of gel for the EEG recording. Some participants suggested the use of wireless caps and dry electrodes in order to make the EEG recording more comfortable and practical for its regular use at home. Future BCI developments for severely impaired people should take these suggestions from real BCI end-users into consideration. Finally, most of participants felt pleased taking part in the study. Actually, they positively rated the ideas of using the proposed BCI system again and participating in new studies. 


\subsection{Limitations}

The results of the present study show that severely disabled people could properly manage assistive P300-based BCI tools improving thus their autonomy and quality of life. However, certain limitations must be noted. First, although the population of this study is large when compared to other BCI studies, especially to studies that involve impaired people, future efforts should be addressed to increase the population under study. It would be desirable that more potential BCI end-users, such as people paralyzed by ALS, MS and/or tetraplegia, took part in future studies. Second, the scope of electronic devices that the proposed BCI tool can operate is quite large since it includes typical devices used in the domestic environment. Therefore, further work will attempt to include new devices and functionalities into the assistive BCI tool. Other remote control protocols such as Bluetooth, Wi-Fi or home automation standards could be explored as new control interfaces. Hence, more demands of end-users at home could be satisfied. Third, recent algorithmic improvements, such as dynamic stopping of stimulation or asynchronous approaches, could enhance regular use of P300-based BCIs. Stopping methods, both fixed and dynamic, would be useful in making P300based BCIs more efficient (Schreuder et al. 2013). Additionally, asynchronous operation allows a more autonomous use of BCI systems by severely impaired people. Aloise et al. (2011a, 2011b) introduced a threshold-based classification approach that allowed eleven healthy volunteers and seven elderly people to divert their attention from the control interface at any time and to increase the accuracy of the system during control periods. Thus, self-paced or asynchronous operation would be more practical for future assistive P300-based BCIs in real life scenarios. Finally, although impaired users might not be able to set up our system without aid of others, the present study took a significant step to assess the degree to which such populations could eventually manage a stand-alone system. 


\section{Conclusions}

7.1. Original contributions

7.2. Conclusions

7.3. Future work 



\subsection{Original contributions}

The main contribution of the Thesis is the research and assessment of an assistive BCI tool for managing electronic devices at home. This is the first research where a large population of potential end-users, individuals with severe disabilities, interact and assess an assistive tool over real devices and inside their own environment. A population of 30 severely impaired subjects took part in this research. The study reports that patients could manage P300-based BCIs independently of their degree of disability, although the ability of attentional control could affect their performance.

The publications related to this Thesis are detailed in Appendix B of this document. The original contributions of the Thesis as well as the advantages of the proposed methodology are given in the following points:

1. This is the first study where a large population of severely impaired subjects was recruited. Previous BCI studies, especially those that involve real endusers, used smaller populations. However, a large population allows to obtain more applicable results. To the best of our knowledge, this is the first study over a large population consisted of people with different degree of both motor and cognitive impairments caused by different pathologies.

2. The usual needs of the disabled population were identified. The research was performed by taking into consideration the potential BCI end-users' needs and wishes. Thus, the proposed assistive BCI tool meets the main comfort, communication and entertainment requirements.

3. In this Thesis, an assistive P300-based BCI tool for operating electronic devices at home was studied. This novel application allows to manage 8 devices usually present at home: TV, DVD player, Hi-Fi system, multimedia drive, lights, heater, fan and phone. Devices were reached by means of IR control commands, using an IR emitter device. The application consisted of a total of 137 commands (115 IR control commands and 22 navigation commands) arranged into 10 matrices of images (the main menu and nine sub-menus). Moreover, the developed assistive tool uses the typical RC stimuli pattern approach using dimming stimuli instead of flashing ones, since it was experimentally showed that dimming stimuli 
were more prominent and patients reported that visual annoyance was decreased.

4. The methodology applied in this Thesis included an initial evaluation of all participants. The degree of motor impairment, cognitive disability and sustained attention ability were assessed by the neuropsychologists of the NRC-D\&D for the entire population (30 subjects). Furthermore, the second group of patients (15 individuals) additionally performed the Luria AND test, and scores for nine cognitive functions were stored: visual perception, spatial orientation, receptive speech, expressive speech, immediate memory, logical memorization, thematic draws, conceptual activity and attentional control. To the best of our knowledge, this is the first study where an exhaustive evaluation of the patients' cognitive functions was carried out.

5. This Thesis explores the online BCI performance of a disabled population in a real environment as strategy to find out whether people with severe disabilities could eventually manage a P300-based BCI at home. The methodology consisted of three experimental sessions: the first one for calibrating and adjusting the classifier for each individual; and two more sessions for assessing their performance with the proposed tool. This methodology allowed to verify that most of patients were able to operate the assistive BCI tool with online accuracy higher $80 \%$.

6. The comparison between the online BCI performance and the degree of impairment and Luria scores of the population under study reinforces the results of previous studies carried out with smaller populations. The results of this Thesis confirm the results of Nijboer et al. (2008) since statistical significance between the users' performance and their degree of motor or cognitive impairment was not reached. Nevertheless, statistical significance was found between the Luria score for the cognitive function of attentional control and the accuracy achieved during the evaluation session. The attention ability usually depends on the specific pathology and stage of each patient. Thus, these results also agree with the suspicion of Piccione et al. (2006) that observed the users' performance could be affected by the severity of the impairment. 
7. In this Thesis not only the design but also the experimental procedure and the evaluation were carried out through real end-users. An assessment questionnaire was included after having finished the experimental stage in order to each individual would rate the proposed BCI tool as an assistive daily tool at home. This Doctoral Thesis takes a noteworthy step considering the opinion and feelings of end-users as well as their degree of satisfaction, since the point of view of real end-users lacked in previous studies involving disabled populations.

\subsection{Conclusions}

A novel assistive tool was investigated for assessing the ability of severely impaired individuals managing a P300-based BCI system at home. The proposed tool for environment control allows to operate 8 real devices, present in the usual environment, by means of 115 IR control commands and 22 navigation commands. It was assessed by a population of 30 potential BCI end-users with different pathologies and degree of both motor and cognitive impairments. These individuals interacted during three sessions managing real devices with the assistive tool in their usual environment. Twenty three out of the thirty subjects were able to properly control the proposed tool with accuracy above $80 \%$. Nineteen out of them even reached accuracy above 95\%. Furthermore, maximum rates up to 6.8 item/min and $25.9 \mathrm{bit} / \mathrm{min}$ were achieved. The analysis of the results suggests the degree of impairment is not a relevant issue in order to properly manage the P300-based BCI tool. Therefore, it could be suitable to assist severely disabled individuals, increasing their personal autonomy at home. Moreover, the proposed tool could straightforwardly increase and adapt the scope of needs that can be satisfied. Hence, the dependence from caregivers, relatives and nurses could be reduced.

In addition to this global conclusion, the results of this research lead to other specific conclusions:

1. The needs identified for the population under study were related to three main categories: comfort, communication and security. Firstly, comfort requirements such as environment control, hygiene and mobility needs were 
identified. Secondly, communication requirements were related to accessibility and entertainment. Thirdly, telecare services, fall detectors at home and management of systems for access control were demanded as security requirements.

2. P300-based BCI are the most proper type of BCI for severely disabled populations since it does not require training period and its typical paradigm allows to select in a fast manner the desired item among a large number of items only by focusing on it. Specifically, the assistive BCI tool proposed in this study provide up to 115 IR commands for managing electronic devices at home and 22 navigation commands. These commands were distributed among ten matrices (the main menu and nine sub-menus) in order to simplify the interface.

3. P300-based BCIs are suitable for assistive applications since disabled populations seem to be able to properly manage them. In this study, twenty three out of thirty individuals were able to operate the proposed tool with accuracy above 80\%; six out of the remaining users reached accuracy lower than 70\%; and for the remaining participant it was not possible to derive a reliable classifier. These results show that most of end-users are able to manage typical P300-based BCIs without special considerations.

4. The degree of impairment (motor or cognitive) does not to have an influence on the performance of individuals managing the proposed assistive tool. Statistical significance was not reached $(p>0.05)$ in the correlation between the online BCI performance and the degree of motor impairment, cognitive impairment and sustained attention ability of the thirty participants. Therefore, these applications are appropriate for assisting and increasing the personal autonomy of severely impaired subjects at home.

5. The analysis of the results showed a significant relation between the users' online BCI performance operating the assistive tool and their Luria AND score for the attentional control function. Nevertheless, Luria AND scores were only available for the second group of patients composed of 15 individuals, whereas the sustained attention ability degree that was provided for both groups of patients (30 subjects) did not show significant relation 
with the users' performance. This result shows that the attention ability of patients is probably the only requirement for using a P300-based BCI, independently of the specific pathology and the degree of impairment.

6. The end-users' assessment carried out by means of a questionnaire showed that BCI potential users are willing to take part in this kind of studies, providing their requirements and their point of view. This should lead us to focus on end-users for designing, developing and assessing future assistive BCI applications.

7. Disabled population showed its satisfaction with the proposed assistive BCI tool through the assessment questionnaires. Most of the participants rated favourably the design and usefulness of the assistive tool for daily use at home.

8. Considering the assessment questionnaire responses, individuals stated that EEG-based BCIs are not practical on a daily basis. It was essentially because of the need of using gel for the EEG recordings. Participants suggested the use of wireless caps and dry electrodes in order to make EEG recordings more comfortable and practical for its regular use at home. Future BCI developments for disabled populations should take these suggestions from real BCI end-users into consideration.

\subsection{Future work}

In this Thesis, an assistive BCI application for operating home devices was developed and then assessed by means of a large population of real end-users. The aim of the research was to prove whether severely impaired people could manage P300based BCIs in their daily life. The results show that most patients could properly operate the proposed assistive tool increasing their personal autonomy at home. Despite of these promising results, there are some points that can be addressed in future work.

Increasing the size of the population should be considered. The developed BCI tool and the proposed methodology should be validated in a larger database to confirm the results shown in this Thesis. Although the population in this study is larger 
than populations in previous studies involving disabled people, further work should be addressed to increase the population under study. Moreover, it would be desirable to include potential end-users with other pathologies, such as individuals paralyzed by ALS, MS and/or tetraplegia, in future studies.

The proposed tool meets the main identified requirements related to comfort, communication and entertainment. Since the scope of possible electronic devices to be managed at home is quite large, future efforts will attempt to include new devices and functionalities into the assistive tool. Hence, more needs of end-users at home could be fulfilled. Moreover, other remote control protocols such as Bluetooth, Wi-Fi or home automation standards should be explored as new control interfaces increasing the possibilities of the BCI tool for assisting at home.

The large database created in this Thesis could be useful to investigate new real-time classification algorithms and the most effective channels for recording EEG signals in P300-based BCIs. Furthermore, it could be suitable to analyse what specific potentials take part in the custom classifiers derived for each individual and whether N2PC potentials could provide complementary information to the P300 potentials for some users. Finally, in order to improve the regular use of P300-based BCIs further efforts should be addressed to include novel signal processing algorithms, such as dynamic stopping of stimulation or asynchronous approaches. On one hand, dynamic stopping methods would make P300-based BCIs more efficient (Schreuder et al. 2013). On the other hand, asynchronous operation would allow a more autonomous use of BCI systems by severely disabled individuals. Threshold-based classification approaches proposed by Aloise et al. (2011a, 2011b) allowed healthy volunteers and elderly subjects to divert their attention from the control interface at any time and to increase the accuracy of the system during control periods. Therefore, self-paced or asynchronous approaches would be recommendable for future assistive P300-based BCIs in real life scenarios. 


\section{Appendix A. Glossary of terms}

\begin{tabular}{|c|c|}
\hline $2 \mathrm{D}$ & 2 dimensions \\
\hline ACC & Accuracy \\
\hline ALS & Amyotrophic lateral sclerosis \\
\hline AND & Adult neuropsychological diagnosis \\
\hline BCI & Brain computer interface \\
\hline CAR & Common averaged reference \\
\hline $\mathrm{CB}$ & Checkerboard stimuli pattern \\
\hline ECoG & Electrocorticogram \\
\hline EEG & Electroencephalogram \\
\hline ER & Emergency \\
\hline ERD & Event-related desynchronization \\
\hline ERP & Event-related potential \\
\hline ERS & Event-related synchronization \\
\hline Eval1 & Evaluation session 1 \\
\hline Eval2 & Evaluation session 2 \\
\hline fMRI & Functional magnetic resonance imaging \\
\hline fNIRS & Functional near-infrared resonance spectroscopy \\
\hline FT & Finished tasks \\
\hline GND & Ground \\
\hline GSVM & Gaussian kernel support vector machine \\
\hline $\mathrm{Hi}-\mathrm{Fi}$ & High-fidelity \\
\hline IMSERSO & Instituto de mayores y servicios sociales \\
\hline
\end{tabular}


Social security administration body responsible for handling social services which deals with older and dependent people

IR

ITR

LDA

LSVM

MI

MEG

MRS

MS

NRC-D\&D

PCM

PET

RC

SCPs

SNR

SPECT

SQUIDs

SR

SSVEPS

SWLDA

TV

VEPs
Infrared

Information transfer rate

Linear discriminant analysis

Linear support vector machine

Motor imagery

Magnetoencephalogram

Magnetic resonance spectroscopy

Multiple sclerosis

National reference centre on disability and dependence

Pearson's correlation method

Positron emission tomography

Row/column stimuli pattern

Slow cortical potentials

Signal to noise ratio

Single photon emission computed tomography

Superconducting quantum interference devices

Selection rate

Steady-state visual evoked potentials

Stepwise linear discriminant analysis

Television

Visual evoked potentials 


\section{Appendix B. Publications}

B.1. Peer-Reviewed International Articles in Journals Indexed in the Journal Citations Report

B.2. Peer-Reviewed Articles in not indexed journals

B.3. Contributions to International Conferences

B.4. Contributions to National Conferences 



\section{B.1. Peer-Reviewed International Articles in Journals Indexed in the Journal Citations Report}

- Luis Fernando Nicolás-Alonso, Rebeca Corralejo, Javier Gómez-Pilar, Daniel Álvarez, Roberto Hornero, "Adaptive Stacked Generalization for Multiclass Motor Imagery-based Brain Computer Interfaces", IEEE Transactions on Neural Systems and Rehabilitation Engineering, vol. 23, 4, pp. 702-712, 2015.

- Luis Fernando Nicolás-Alonso, Rebeca Corralejo, Javier Gómez-Pilar, Daniel Álvarez, Roberto Hornero, "Adaptive semi-supervised classification to reduce intersession non-stationarity in multiclass motor imagery-based brain computer interfaces", Neurocomputing, vol. 159, 2, pp. 186-196, 2015.

- Rebeca Corralejo, Luis F. Nicolás-Alonso, Daniel Álvarez, Roberto Hornero, "A P300-based brain-computer interface aimed at operating electronic devices at home for severely disabled people", Medical and Biological Engineering and Computing, vol. 52, 10, pp. 861-872, 2014.

- Jesús Poza, Carlos Gómez, María García, Rebeca Corralejo, Alberto Fernández, Roberto Hornero, "Analysis of neural dynamics in mild cognitive impairment and Alzheimer's disease using the wavelet turbulence", Journal of Neural Engineering, vol. 11, 2, pp. 026010, 2014.

\section{B.2. Peer-Reviewed Articles in not indexed journals}

- Roberto Hornero, Rebeca Corralejo, Daniel Álvarez, Laura Martín, "Diseño, desarrollo y evaluación de un sistema Brain Computer Interface (BCI) aplicado al control de dispositivos domóticos para mejorar la calidad de vida de las personas con grave discapacidad", Trauma, vol. 24, 2, pp. 117-125, 2013.

- Rebeca Corralejo, Roberto Hornero, Daniel Álvarez, Laura Martín, "Improving the accessibility at home: implementation of a domotic 
application using a P300-based brain computer interface system", Journal of Accessibility and Design for All, vol. 2, 1, pp. 1-14, 2012.

- Roberto Hornero, Rebeca Corralejo, Daniel Álvarez, "Brain Computer Interface (BCI) aplicado al entrenamiento cognitivo y control domótico para prevenir los efectos del envejecimiento", LYCHNOS - Cuadernos de la Fundación General CSIC, vol. 8, pp. 29-34, 2012.

\section{B.3. Contributions to International Conferences}

- Javier Gómez-Pilar, Rebeca Corralejo, Luis Fernando Nicolas-Alonso, Daniel Álvarez, Roberto Hornero, "Assessment of Neurofeedback Training by Means of Motor Imagery Based-BCI for Cognitive Rehabilitation", 36th Annual International Conference of the IEEE Engineering in Medicine and Biology Society Conference, Proceedings of the 36th Annual International Conference of the IEEE Engineering in Medicine and Biology Society Conference, pp. 3630-3633, Chicago (EE.UU.), 2014.

- Luis F. Nicolas-Alonso, Rebeca Corralejo, Javier Gómez-Pilar, Daniel Álvarez, Roberto Hornero, "Ensemble learning for classification of motor imagery tasks in multiclass brain computer interfaces", 6th Computer Science and Electronic Engineering Conference (CEEC), 6th Computer Science and Electronic Engineering Conference (CEEC), pp. 79, Colchester (Reino Unido), 2014.

- Rebeca Corralejo, Luis Fernando Nicolás-Alonso, Daniel Álvarez, Roberto Hornero, "Assessment of an Assistive P300-based Brain Computer Interface by Users with Severe Disabilities", XIII Mediterranean Conference on Medical and Biological Engineering and Computing (MEDICON 2013), IFMBE Proceedings of the XIII Mediterranean Conference on Medical and Biological Engineering and Computing, vol. 41, pp. 1647-1650, Sevilla (España), 2013.

- Luis F. Nicolas-Alonso, Rebeca Corralejo, Daniel Álvarez, Roberto Hornero, "Adaptive Classification Framework for Multiclass Imagery-based 
BCI", XIII Mediterranean Conference on Medical and Biological Engineering and Computing (MEDICON 2013), IFMBE Proceedings of the XIII Mediterranean Conference on Medical and Biological Engineering and Computing, vol. 41, pp. 762-765, Sevilla (España), 2013.

- Luis .F. Nicolas-Alonso, Rebeca Corralejo, Daniel Álvarez Member, Roberto Hornero, "Analytic Common Spatial Pattern and Adaptive Classification for Multiclass Motor Imagery-based BCI", 6th Internantional IEEE EMBS Conference on Neural Engineering (NER), Conference on Neural Engineering (NER), 2013 6th International IEEE/EMBS, pp. 10841087, San Diego (EEUU), 2013.

- Rebeca Corralejo, Luis Fernando Nicolás-Alonso, Daniel Álvarez, Roberto Hornero, "Development of a P300-based Brain Computer Interface to Assist Severe Disabled People", V Congreso Internacional de Diseño, Redes de Investigación y Tecnología para todos (DRT4All 2013), Libro de Actas del V Congreso Internacional de Diseño, Redes de Investigación y Tecnología para todos, pp. 160-165, Madrid (España), 2013.

- Rebeca Corralejo, Daniel Álvarez, Roberto Hornero, "A P300-based BCI Aimed at Managing Electronic Devices for People with Severe Disabilities", International Conference on NeuroRehabilitation (ICNR 2012), Part I, Biosystems \& Biorobotics, Converging Clinical and Engineering Research on Neurorehabilitation, pp. 641-645, Toledo (España), 2012.

- Rebeca Corralejo, Roberto Hornero, Daniel Álvarez, "Feature Selection Using a Genetic Algorithm in a Motor Imagery-Based Brain Computer Interface", 33rd Annual International Conference of the IEEE Engineering in Medicine and Biology Society, Proceedings of the 33rd Annual International Conference of the IEEE Engineering in Medicine and Biology Society, pp. 7703-7706, Boston (EE.UU.), 2011.

- Rebeca Corralejo, Daniel Álvarez, Roberto Hornero, "Aplicación domótica mediante un sistema Brain Computer Interface basado en potenciales P300", VI Congreso Iberoamericano de Tecnologías de Apoyo a la Discapacidad, Libro de Actas del VI Congreso Iberoamericano de Tecnologías de Apoyo a 
la Discapacidad, Volumen II, pp. 111-117, Palma de Mallorca (Spain), 2011.

- Rebeca Corralejo, Roberto Hornero, Daniel Álvarez, "A Domotic Control System Using Brain-Computer Interface (BCI)", 11th International WorkConference on Artificial Neural Networks (IWANN 2011), Proceedings, Part I, Lecture Notes in Computer Science vol. 6691, pp. 345-352, Torremolinos (Spain), 2011.

- Rebeca Corralejo, Roberto Hornero, Daniel Álvarez, Laura Martín, "Improving the Accesibility at Home: Implementation of a Domotic Application using a P300-based Brain Computer Interface System", IV Congreso Internacional de Diseño, Redes de Investigación y Tecnología para todos (DRT4ALL 2011), Libro de Actas del IV Congreso Internacional de Diseño, Redes de Investigación y Tecnología para todos, pp. 75-84, Madrid (Spain), 2011.

\section{B.4. Contributions to National Conferences}

- Javier Gómez-Pilar, Rebeca Corralejo, Víctor Martínez-Cagigal, Daniel Álvarez, Roberto Hornero, "Análisis de los cambios espectrales del EEG producidos por el entrenamiento neurocognitivo mediante una interfaz cerebro-ordenador", $7^{\circ}$ Simposio CEA de Bioingeniería 2015, Interfaces Cerebro-Computadora (BCI) Tecnologías Asistenciales, Libro de Actas del Simposio CEA de Bioingeniería 2015, pp. 15-21, Málaga (España), 2015.

- Javier Gómez-Pilar, Rebeca Corralejo, Luis Fernando Nicolás-Alonso, Daniel Álvarez, Roberto Hornero, "Diseño y desarrollo de una plataforma de entrenamiento cognitivo basada en BCI para prevenir los efectos del envejecimiento", $6^{\circ}$ Simposio CEA de Bioingeniería 2014, Interfaces Mente Computador y Neurotecnologías, Libro de Actas del Simposio CEA de Bioingeniería 2014, pp. 25-31, Granada (España), 2014.

- Rebeca Corralejo, Daniel Álvarez, Roberto Hornero, "Evaluación de un sistema BCI de control domótico basado en potenciales P300 aplicado a 


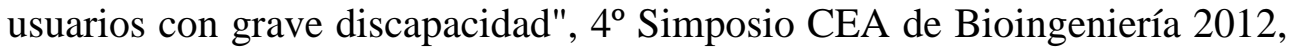
BCI (Brain Computer Interface) y Tecnologías de la rehabilitación, Libro de Actas del Simposio CEA de Bioingeniería 2012, pp. 11-18, Valladolid (España), 2012.

- Laura Martín, Rebeca Corralejo, Roberto Hornero, "Aplicación de las Máquinas de Vector Soporte en Sistemas Brain Computer Interface basados en potenciales P300", XXX Congreso Anual de la Sociedad Española de Ingeniería Biomédica (CASEIB 2012), Libro de Actas del XXX Congreso Anual de la Sociedad Española de Ingeniería Biomédica (CASEIB 2012), San Sebastián (España), 2012.

- Rebeca Corralejo, Daniel Álvarez, Roberto Hornero, "Análisis de electroencefalogramas en ritmos sensoriomotores. Control domótico mediante Brain Computer Interface", 3er Simposio CEA de Bioingeniería 2011, Técnicas de BCI y Neurotecnologías, Libro de Actas del Simposio CEA de Bioingeniería 2011, pp. 11-17, Palma de Mallorca (España), 2011.

- Rebeca Corralejo, Daniel Álvarez, Roberto Hornero, "Brain Computer Interface aplicado al control de una aplicación domótica", Simposio CEA de Bioingeniería 2010, Libro de Actas del Simposio CEA de Bioingeniería 2010, pp. 28-36, Málaga (España), 2010.

- Rebeca Corralejo, Roberto Hornero, Daniel Álvarez, Luis Miguel San José, "Diseño de un algoritmo genético como método de selección de características en un sistema BCI basado en ritmos sensoriomotores", XXVIII Congreso Anual de la Sociedad Española de Ingeniería Biomédica (CASEIB 2010), Libro de Actas del XXVIII Congreso Anual de la Sociedad Española de Ingeniería Biomédica (CASEIB 2010), pp. 247, Madrid (España), 2010.

- Rebeca Corralejo, Roberto Hornero, Daniel Álvarez, "Desarrollo de una aplicación domótica controlada por Brain Computer Interface (BCI)", XXVII Congreso Anual de la Sociedad Española de Ingeniería Biomédica (CASEIB 2009), Libro de Actas del XXVII Congreso Anual de la Sociedad Española de Ingeniería Biomédica (CASEIB-2009), pp. 577-580, Cádiz (España), 2009. 
- Javier Temprano, Roberto Hornero, Daniel Abásolo, Rebeca Corralejo, "Clasificación de características extraídas del electroencefalograma en sistemas Brain Computer Interface", XXVI Congreso Anual de la Sociedad Española de Ingeniería Biomédica (CASEIB 2008), Libro de Actas del XXVI Congreso Anual de la Sociedad Española de Ingeniería Biomédica (CASEIB 2008), pp. 178-181, Valladolid (España), 2008.

- Rebeca Corralejo, Roberto Hornero, Daniel Abásolo, Javier Temprano, "Comparación de métodos de extracción de características para su aplicación a Brain Computer Interface", XXVI Congreso Anual de la Sociedad Española de Ingeniería Biomédica (CASEIB 2008), Libro de Actas del XXVI Congreso Anual de la Sociedad Española de Ingeniería Biomédica (CASEIB 2008), pp. 174-177, Valladolid (España), 2008. 


\section{Appendix C. Síntesis de la tesis doctoral en castellano}

C.1. Introducción

C.2. Estado del arte

C.3. Metodología

C.4. Aplicación BCI para el control de dispositivos electrónicos presentes en el hogar

C.5. Resultados

C.6. Discusión

C.7. Conclusiones

C.7.1. Contribuciones originales

C.7.2. Conclusiones

C.7.3. Líneas futuras 



\section{C.1. Introducción}

El estudio presentado en la Tesis Doctoral pertenece al ámbito de la Ingeniería Biomédica. Esta disciplina emplea métodos y principios de la ingeniería para abordar problemas propios de la medicina o la biología (Nebeker, 2002). Concretamente, la Tesis se centra en el procesado de señales biomédicas y en aplicaciones de neuroingeniería.

La Tesis propone el estudio de una nueva aplicación de las interfaces cerebro-ordenador o Brain Computer Interface (BCI) para personas con grave discapacidad. Un BCI es un sistema de comunicación que proporciona un canal alternativo a los procesos de comunicación y control naturales, ya que no emplea las conexiones neuromusculares, que son los canales de salida normales del cuerpo humano (Graimann et al. 2010, Wolpaw et al. 2000). Por tanto, un sistema BCI monitoriza la actividad cerebral y traduce características específicas de la misma, relacionadas con la intención del usuario, en comandos que permiten controlar un dispositivo (Wolpaw et al. 2002). Los sistemas BCI son especialmente interesantes en el caso de personas con grave discapacidad que les impide la realización de movimientos. Las tecnologías de comunicación aumentativas y alternativas convencionales necesitan de algún tipo de control muscular y, precisamente por ello, pueden no resultar útiles para aquellas personas con grave discapacidad motora, como los afectados por esclerosis lateral amiotrófica (ELA) en estadio avanzado, por apoplejía o por parálisis cerebral grave (Wolpaw et al. 2000).

Existen varios métodos para registrar la actividad eléctrica cerebral. El método más empleado en los sistemas BCI es el electroencefalograma (EEG). A pesar de que tiene una baja resolución espacial, presenta ventajas importantes frente a otras técnicas de registro puesto que se trata de un método no invasivo, de bajo coste, portátil y sencillo. Estas cualidades hacen que sea más práctica para la mayoría de las aplicaciones BCI que otras técnicas de registro con mejor resolución espacial.

El Grupo de Ingeniería Biomédica inició hace unos años una nueva línea de investigación centrada en sistemas BCI con el objetivo de aplicar sus conocimientos en procesado avanzado de señales biomédicas. El objetivo principal fue el estudio de aplicaciones para usuarios finales potenciales de los sistemas BCI, es decir, personas con grave discapacidad. Para ello, el grupo se puso en contacto con el Centro de 
Referencia Estatal de Discapacidad y Dependencia (National Reference Centre on Disability and Dependence, NRC-D\&D) y a partir de esta colaboración surgió una aplicación BCI orientada a control del entorno. Esta primera aplicación empleaba un sistema BCI basado en imágenes motoras (motor imagery, MI). Este tipo de sistemas se fundamentan en que tanto la preparación para ejecutar un movimiento como la imaginación del mismo, sin llegar a realizarlo, producen un patrón de actividad cerebral similar, reconocible y capaz de ser generado voluntariamente (Graimann et al. 2010, Pineda et al. 2000). Por lo tanto, los sistemas BCI basados en MI se controlan a partir de la capacidad del usuario de modificar su propia actividad cerebral (Wolpaw et al. 2000). A pesar de que los resultados en el laboratorio con sujetos sanos fueron prometedores, las sesiones realizadas con usuarios del centro en su entorno habitual mostraron que estos usuarios presentaban dificultades para controlar el movimiento del cursor en 1D. Los sistemas BCI basados en MI requieren de una alta capacidad de concentración para controlar el cursor y en el caso de los usuarios finales este requisito supuso un problema, ya que habitualmente además de discapacidad motora, los usuarios también presentan algún grado de discapacidad cognitiva. Además, se observó que el registro de la actividad cerebral empleando la técnica del EEG con electrodos pasivos requería de mucho tiempo de preparación y esto influía negativamente en la motivación de los usuarios y en su rendimiento posterior con la aplicación BCI.

La presente Tesis propone el uso de una aplicación BCI de control del entorno basada en potenciales evocados P300. El objetivo consiste en investigar si los sistemas BCI basados en P300 son adecuados para que las personas con grave discapacidad puedan controlar apropiadamente los dispositivos presentes en su entorno habitual. Para ello, se ha empleado una metodología centrada en el usuario final de forma que tanto el diseño, como la etapa experimental y la evaluación se han llevado a cabo considerando la opinión de los usuarios finales. Por ello, la población bajo estudio está formada por potenciales usuarios finales de los sistemas BCI, es decir, personas con grave discapacidad. Además, los experimentos se han realizado empleando dispositivos reales y han tenido lugar en el entorno habitual del usuario. Por último, han sido los propios participantes quienes han evaluado la aplicación propuesta. A diferencia de los sistemas basados en MI, este tipo de sistemas no requieren de una etapa de entrenamiento ni de un alto nivel de concentración por lo que se espera que sean más apropiado para las personas con discapacidad. Además, el paradigma P300 permite 
presentar al usuario un número mayor de dispositivos a controlar así como de funcionalidades de los mismos.

\section{C.2. Estado del arte}

La técnica del EEG se emplea habitualmente para registrar la actividad cerebral en los sistemas BCI, ya que es sencilla y de bajo coste (Corralejo et al. 2014, Wolpaw et al. 2000). Los sistemas BCI detectan y clasifican patrones específicos de actividad en las señales cerebrales relacionadas con tareas o eventos concretos (Graimann et al. 2010). Según la naturaleza de las señales de entrada, los sistemas BCI basados en EEG se dividen en dos tipos. Por un lado, los sistemas BCI endógenos, que dependen de la capacidad del usuario para controlar su propia actividad electrofisiológica, como la amplitud en una banda de frecuencia específica del EEG sobre una zona concreta de la corteza cerebral (Wolpaw et al. 2000). Los sistemas BCI basados en MI o en potenciales corticales lentos (slow cortical potentials, SCP) son sistemas endógenos y, a menudo, requieren de una etapa de entrenamiento prolongada. Por otro lado, los sistemas BCI exógenos dependen de actividad electrofisiológica originada externamente, es decir, evocada por estímulos externos concretos. Estos sistemas no necesitan un entrenamiento intensivo pero su funcionamiento requiere de un entorno más estructurado (Wolpaw et al. 2000). Los sistemas BCI basados en potenciales evocados P300 o en potenciales evocados visuales de estado estable (steadystate visual evoked potentials, SSVEP) son sistemas exógenos.

Los sistemas BCI basados en potenciales evocados P300 emplean habitualmente estímulos visuales. Un BCI basado en P300 consiste en elementos dispuestos en una matriz que se iluminan (estímulos) en una secuencia aleatoria. Típicamente estos elementos suelen ser letras pero también podrían ser símbolos o imágenes representando otras opciones (Bell et al. 2008, Citi et al. 2008). La atención selectiva al símbolo deseado, hace que cuando se ilumina dicho símbolo, aparezca un potencial evocado en la actividad cerebral. Dicho potencial se conoce como P300 y se origina en el área centro-parietal de la corteza cerebral, aproximadamente unos $300 \mathrm{~ms}$ después de haberse producido el estímulo. La principal ventaja de los sistemas basados en P300 es que no necesitan entrenamiento y, además, son bastante robustos (Wolpaw 
et al. 2002). Esta Tesis propone el uso de sistemas BCI basados en P300 para obtener aplicaciones asistivas que resulten prácticas en el hogar para las personas con grave discapacidad, ya que no necesitan etapa de entrenamiento y permiten seleccionar de forma rápida la opción deseada frente a un amplio número de opciones presentadas.

La investigación en sistemas BCI basados en P300 se ha llevado a cabo desde diferentes ámbitos. Algunos estudios se han centrado en mejorar los algoritmos de procesado de señal (Aloise et al. 2011a, Khan et al. 2012, Krusienski et al. 2006, Rakotomamonjy and Guigue 2008, Rivet et al. 2009, Serby et al. 2005, Xu et al. 2004). Otros trabajos han analizado el efecto de diferentes paradigmas de presentación de estímulos visuales (Jin et al. 2011, Jin et al. 2012, Mak et al. 2012, Salvaris and Sepulveda 2009, Sellers et al. 2006). Sin embargo, otros estudios han investigado otro tipo de estímulos, como los auditivos (Klobassa et al. 2009) o táctiles (Brouwer and van Erp 2010). Finalmente, algunos trabajos se han enfocado al desarrollo de nuevas aplicaciones de los sistemas BCI basados en P300 (Citi et al. 2008, Corralejo et al. 2014, Finke et al. 2009, Iturrate et al. 2009, Mugler et al. 2010, Yu et al. 2012).

Las aplicaciones BCI basadas en P300 desarrolladas hasta la fecha se han orientado a la comunicación (deletreadores o spellers), a la navegación por Internet, al control de sillas de ruedas, al entretenimiento o al control de entorno. A pesar de que los potenciales usuarios finales de estos sistemas son las personas con grave discapacidad, la mayoría de los estudios de sistemas BCI se evalúan con sujetos sanos. No obstante, algunos autores sí que han contado con los usuarios finales a la hora de profundizar en los sistemas BCI basados en P300. En concreto, se han realizado estudios enfocados a poblaciones de usuarios finales de deletreadores o spellers (Mak et al. 2012, Nijboer et al. 2008, Ortner et al. 2011, Sellers and Donchin 2006, Spüler et al. 2012); y de aplicaciones de control del entorno virtual o simulado (Aloise et al. 2011b, Hoffman et al. 2008).

\section{C.3. Metodología}

La población bajo estudio estuvo formada por 30 sujetos con discapacidad motora, causada por diferentes patologías. Algunos participantes, presentaban también discapacidad cognitiva. Todos los sujetos eran usuarios del NRC-D\&D y no tenían 
experiencia previa en sistemas BCI basados en P300. El estudio fue aprobado por el comité ético del centro y todos los sujetos dieron su consentimiento informado para participar en el estudio. Los participantes fueron reclutados en dos etapas. Primero, un grupo de 15 sujetos y, después, un segundo grupo de otros 15 sujetos. Así, la población bajo estudio consistió en 30 sujetos: 18 hombres y 12 mujeres, con una edad media de $48.20 \pm 10.50$ años y con un rango de edad comprendido entre 26 y 67 años. Los terapeutas del NRC-D\&D valoraron el grado discapacidad motora, de discapacidad cognitiva y la capacidad de atención sostenida de cada participante. Además, los 15 sujetos del segundo grupo realizaron el test de Luria, que valora 9 funciones distribuidas en 5 áreas: visoespacial (percepción visual y orientación espacial), lenguaje oral (habla receptiva y habla expresiva), memoria (memoria inmediata y memorización lógica), inteligencia (imágenes temáticas y actividad conceptual) y atención (control atencional).

La señal de EEG de cada participante fue registrada durante las sesiones experimentales. Se registraron 8 canales (Fz, Cz, P3, Pz, P4, PO7, PO8 y Oz) de acuerdo al sistema internacional 10-20 modificado (Jasper 1958, Jurcak et al. 2007). La distribución de electrodos propuesta cubre la corteza central, parietal y occipital del cerebro, permitiendo el registro de los potenciales P300 así como de potenciales adicionales evocados por los estímulos visuales (Schalk 2007). Para el registro de la señal EEG se empleó un equipo formado por un amplificador g.USBamp y electrodos activos g.LADYbird de g.tec (Guger Technologies OG, Graz, Austria). Se tomó como referencia el lóbulo de la oreja derecha y como tierra el canal Fpz. La frecuencia de muestreo empleada fue de $256 \mathrm{~Hz}$. Se aplicó un filtrado paso banda entre 0.1 y $60 \mathrm{~Hz}$, así como un filtro de ranura a $50 \mathrm{~Hz}$ para eliminar la componente de la red eléctrica. El registro de las señales y el diseño de los experimentos se controló mediante la herramienta de propósito general BCI2000 (Schalk et al. 2004, Schalk and Mellinger 2010).

El paradigma P300 presenta al usuario una matriz con diferentes elementos. Típicamente, los elementos de la matriz van iluminándose, agrupados por filas y columnas, aleatoriamente. El usuario ha de fijarse en el símbolo que desea seleccionar y contar cuántas veces se ilumina éste. Cada vez que se ilumine la fila o la columna que contienen el símbolo deseado (estímulos atendidos) aparecerá en la actividad cerebral del usuario un potencial evocado P300. De esta forma, es posible detectar la fila y la columna que evocan dicho potencial y, por tanto, en la intersección de ambas se 
encontrará el símbolo deseado. El procesado de señal necesario para detectar los estímulos atendidos incluye 3 etapas: extracción, selección y clasificación de características. En primer lugar, durante la fase de extracción de características típicamente se aplica un filtrado espacial y temporal. En el presente estudio, se ha empleado un referencia de media común (common average reference, CAR) como filtro espacial. El filtrado temporal se ha realizado almacenando y promediando separadamente para cada estímulo concreto las épocas de 800 ms registradas inmediatamente después de dicho estímulo. En segundo lugar, a partir de los datos obtenidos durante la primera sesión experimental, se aplica un análisis discriminante lineal paso a paso (stepwise linear discriminant analysis, SWLDA). Este método permite seleccionar las características (valor de amplitud para un canal y un instante de tiempo específico) que permiten discriminar mejor entre las épocas que contienen una respuesta evocada (y, por tanto, se corresponden con un estímulo atendido) y las que no. Este proceso se aplica de forma independiente para cada usuario. Así, se obtiene una función discriminante que se aplica, posteriormente, en la etapa de clasificación. La salida del clasificador refleja la probabilidad de cada fila y columna de ser estímulos atendidos (Schalk and Mellinger 2010). De esta forma, es posible determinar el símbolo deseado por el usuario identificando el elemento situado en la intersección de la fila y la columna que obtienen el valor de probabilidad más alto.

\section{C.4. Aplicación BCI para el control de dispositivos electrónicos presentes en el hogar}

En el presente estudio, para evaluar la adecuación de los sistemas BCI basados en P300 a las personas con grave discapacidad, se desarrolló una aplicación BCI asistiva de control de dispositivos electrónicos en el hogar. En la etapa de diseño de la aplicación participaron tanto usuarios finales de los sistemas BCI: usuarios del NRCD\&D, como el personal de dicho centro. Todas las necesidades detectadas se agruparon en tres grandes grupos: confort (entorno, higiene, movilidad); comunicación (relaciones, accesibilidad, entretenimiento); y seguridad (asistencia, control de accesos). Estas necesidades se tuvieron en consideración a la hora de desarrollar la aplicación BCI propuesta en el estudio. Así, ésta satisface necesidades de confort, comunicación, 
accesibilidad y entretenimiento, a partir del control de 8 dispositivos electrónicos: TV, reproductor de DVD, equipo de música, disco multimedia, luces, calefactor, ventilador y teléfono.

La aplicación BCI asistiva propuesta en este estudio se basa en el paradigma odball, en el que estímulos atendidos pero infrecuentes mezclados con estímulos frecuentes no atendidos provocan la aparición de potenciales P300 en la actividad cerebral del usuario. La aplicación propuesta emplea matrices de imágenes, en lugar de la matriz típica de caracteres (Donchin et al. 2000, Farwell and Donchin 1988, Nijboer et al. 2008). Además, en lugar de producir los estímulos iluminando los elementos éstos se atenúan, ya que se comprobó experimentalmente que para estas imágenes el contraste se apreciaba mejor y resultaban menos molestos para los usuarios (Corralejo et al. 2014). La aplicación está compuesta por un menú principal y nueve sub-menús. Al comienzo, se muestra al usuario el menú principal de la aplicación. Éste consiste en una matriz de 3 x 4 elementos cuyas dos primeras filas contienen imágenes que representan los dispositivos que el usuario puede controlar. En la última fila se muestra el acceso a la agenda telefónica, así como tres comandos de control: pausar, reanudar y parar la ejecución de la aplicación. Al igual que en el paradigma típico de P300 (Donchin et al. 2000), para realizar una selección se muestran al usuario 15 secuencias aleatorias de estímulos. Cada secuencia engloba la atenuación de cada fila y columna de la matriz. Los estímulos se suceden aleatoriamente cada 187.5 ms: cada fila/columna se atenúa durante $62.5 \mathrm{~ms}$ y a continuación la matriz se mantiene invariante durante $125 \mathrm{~ms}$ (Furdea et al. 2009, Kleih et al. 2010, Spüler et al. 2012). Los usuarios tienen que fijarse en el elemento que desean seleccionar y contar cuántas veces se atenúa. Una vez que todas las secuencias de estímulos han terminado y se ha determinado qué elemento del menú principal es el deseado, se ejecuta la acción correspondiente a dicho elemento: acceder al sub-menú de un dispositivo específico o pausar, reanudar o parar la ejecución de la aplicación. De esta forma, desde el menú principal los usuarios pueden acceder a los sub-menús, que contienen tanto las funcionalidades específicas de cada dispositivo como la opción de volver al menú principal. Los sub-menús presentan matrices de imágenes de tamaño variable ( 3 x 4, 4 x 4 ó 5 x 5), en función del número de funcionalidades disponibles para cada dispositivo, y con un color de fondo diferente. El Capítulo 4 del presente documento presenta varias figuras que muestran la apariencia de los menús de la aplicación. Al igual que para el menú principal, las filas y columnas de 
la matriz de cada sub-menú se atenúan aleatoriamente. El usuario se tiene que fijar en la nueva opción deseada y contar mentalmente cuántas veces se atenúa. Una vez identificado el elemento deseado, se ejecuta el comando seleccionado (ej. encender el ventilador, subir el volumen, llamar a un contacto de la agenda telefónica o volver al menú principal). Así, los usuarios pueden navegar a través de la aplicación BCI y controlar estos dispositivos presentes comúnmente en el hogar. Los comandos de control de cada dispositivo se encuentran almacenados en una base de datos de señales de infrarrojos (IR). Para ejecutar estos comandos se emplea un dispositivo emisor de IR RedRat3-II (RedRat Ltd., Cambridge, UK) que se conecta al ordenador a través de un puerto USB.

Los participantes realizaron tres sesiones experimentales con la aplicación en su entorno habitual y empleando dispositivos reales. El procedimiento consistió en la realización de una sesión de calibración y dos sesiones de evaluación de la aplicación BCI. Durante la sesión de calibración cada usuario realizó 10 rondas guiadas de 5-6 selecciones, con 15 secuencias de estímulos y empleando para ello el sub-menú de la TV (matriz de tamaño $5 \times$ 5). Durante las rondas guiadas se le indica al usuario previamente qué elemento de la matriz ha de seleccionar. La información de las 5 primeras rondas se empleó para generar la función discriminante específica para cada usuario; mientras que las 5 rondas finales se emplearon para validar dicho clasificador. En las sesiones de evaluación (Eval1 y Eval2), los usuarios realizaron inicialmente 2 rondas guiadas de 6 selecciones, para confirmar el correcto funcionamiento de la aplicación (Nijboer et al. 2008). A continuación, los usuarios realizaron al menos 7 rondas navegando libremente a través de todos los menús de la aplicación con el objetivo de completar una serie de al menos 6 selecciones propuesta previamente (ej. acceder al menú TV, encender la TV, cambiar al canal 5, volver al menú principal, acceder al menú DVD, y grabar la emisión actual). Para ello, se empleó el clasificador obtenido durante la sesión de calibración. En la sesión Eval1 se emplearon 15 secuencias de estímulos, mientras que en la sesión Eval2 se redujo el número de secuencias en función de la precisión obtenida por cada usuario. Cuanto menor número de secuencias se necesite, se presentan menos estímulos al usuario, se emplea menos tiempo y, por lo tanto, la selección se realiza más rápido. El nivel de precisión exigido a los participantes fue del 70\% sobre las últimas rondas de la sesión de calibración o sobre las dos primeras rondas de las sesiones de evaluación. Se considera que un 70\% 
es la precisión mínima que garantiza una comunicación efectiva en un sistema BCI (Kleih et al. 2010, Kübler et al. 2001). Por ello, los participantes que no cumplieron este criterio tras la primera sesión, repitieron las tareas de calibración durante la siguiente sesión. Igualmente, los participantes que no alcanzaron el 70\% de precisión durante la primera sesión de evaluación, repitieron las tareas de Eval1 durante la siguiente sesión. Los resultados se midieron en términos de precisión online ACC (porcentaje de elementos deseados identificados correctamente frente al número total de selecciones); tasa o velocidad de selección $S R$ (número de selecciones por minuto); y tasa de transferencia de información ITR (bits transmitidos por minuto).

Finalmente, tras realizar la última sesión experimental, los usuarios completaron un cuestionario de satisfacción para evaluar al aplicación BCI propuesta. Los participantes valoraron 11 sentencias relacionadas con la aplicación en una escala Likert (Allison et al. 2010, Münßinger et al. 2010) de 5 puntos (0-totalmente en desacuerdo, 1-en desacuerdo, 2-neutral, 3-de acuerdo, 4-totalmente de acuerdo). Las sentencias impares eran afirmaciones positivas sobre la aplicación BCI (puntuación ideal: 4), mientras que las pares eran afirmaciones negativas (puntuación ideal: 0).

\section{C.5. Resultados}

La aplicación BCI basada en P300 propuesta en el presente estudio fue evaluada por 30 personas con grave discapacidad. Los participantes llevaron a cabo tres sesiones experimentales con la aplicación, una sesión de calibración y dos de evaluación. Una vez finalizada la etapa experimental completaron un cuestionario de evaluación de la aplicación BCI propuesta.

En primer lugar, respecto a la sesión de calibración, 25 de los 30 participantes obtuvieron valores de precisión ACC superiores al 70\%. El resto de usuarios (P6, P11, P13, P15 and P26) repitieron las tareas de calibración durante la siguiente sesión. En el caso del sujeto P13, no fue posible detectar adecuadamente los potenciales evocados P300 tras haber realizado tareas de calibración durante las tres sesiones experimentales. En segundo lugar, en cuanto a las sesiones de evaluación de la aplicación, 23 de los 29 sujetos que realizaron estas sesiones obtuvieron precisiones de manejo superiores al 80\%. Incluso, 19 de ellos obtuvieron precisiones superiores al 
95\%. El resto de participantes (P5, P6, P7, P11, P15 and P26) no alcanzaron un 70\% de precisión durante la primera sesión de evaluación. Cinco de ellos repitieron las tareas de Eval1 durante la siguiente sesión y todos ellos, excepto P5 y P26, consiguieron valores de precisión más altos. El participante P7 incluso alcanzó un 77\% de precisión tras repetir la sesión Eval1. Por último, respecto a la velocidad de selección $S R$ y la tasa de transferencia ITR, se observó que los participantes que alcanzaron los valores más altos de precisión pudieron manejar la aplicación durante la sesión Eval2 empleando un menor número de secuencias. Por tanto, estos sujetos navegaron más rápido y obtuvieron valores de $S R$ e ITR más altos. En concreto, los valores de velocidad de selección oscilaron entre 2.23 y 6.82 selecciones/min y los de ITR entre 2.19 y 25.91 bit/min, teniendo en cuenta la pausa existente entre selecciones. Mientras que los valores de $S R$ variaron entre 2.87 y 21.49 selecciones/min y los de ITR entre 2.82 y $81.58 \mathrm{bit} / \mathrm{min}$, si no se tiene en cuenta la pausa existente entre una selección y la siguiente.

Finalmente, los participantes evaluaron el diseño, funcionamiento y utilidad de la aplicación mediante un cuestionario de satisfacción. Todas las sentencias impares, afirmaciones positivas, obtuvieron una puntuación media superior a 2 (puntuación ideal. 4); mientras que las sentencias pares, afirmaciones negativas, se valoraron con puntuaciones medias inferiores a 2 (puntuación ideal: 0). Por lo tanto, las puntuaciones indican que los participantes evaluaron favorablemente tanto el diseño como la utilidad de la aplicación BCI propuesta. La única excepción se dio para la afirmación 6, respecto a la comodidad de los registros EEG en el caso de un uso diario de la aplicación, que obtuvo una puntuación media superior a 2.

\section{C.6. Discusión}

En el presente trabajo se estudió una nueva aplicación BCI asistiva basada en P300 que fue evaluada por un población de 30 personas con grave discapacidad. 23 de los 30 participantes controlaron la aplicación con una precisión superior al 80\%. Incluso, 19 de ellos alcanzaron precisiones superiores al 95\%. Estos resultados son notables puesto que se trata de potenciales usuarios finales de las aplicaciones BCI. 3 de los 6 usuarios restantes (P6, P11 y P15) obtuvieron precisiones entre 60 y 65\%, 
mientras que los otros 3 participantes (P5, P13 y P26) no consiguieron un control aceptable de la aplicación. En el caso del usuario P13, tras haber realizado tareas de calibración durante tres sesiones, no fue posible discriminar los estímulos atendidos de los no atendidos. Esto fue debido a la pobre calidad de las señales EEG registradas, que presentaban múltiples artefactos causados por espasmos musculares. Además, si se tiene en cuenta a los participantes con mayor grado de discapacidad (P5, P10, P11, P14, P15 and P21), los que presentan discapacidad motora grave o moderada junto con algún grado de discapacidad cognitiva, se observa que estos alcanzaron resultados durante las sesiones de evaluación que variaron de modestos (63\%) a excelentes (100\%). El usuario P5 fue el único de ellos que no pudo controlar adecuadamente la aplicación BCI.

Para identificar si el tipo o el grado de discapacidad puede influir en el manejo de estos sistemas, se analizó la correlación entre el rendimiento de los usuarios durante las sesiones experimentales y las valoraciones realizadas a los participantes. No se encontró relación entre los resultados obtenidos por los usuarios y su grado de discapacidad motora $(r=0.1961, p=0.2991)$, de discapacidad cognitiva $(r=$ $0.2229, p=0.2364)$ y de capacidad de atención sostenida $(r=0.3337, p=0.0715)$. Estos resultados coinciden con los obtenidos por Nijboer et al. (2008) en estudios previos, puesto que no encontró relacion entre el grado de discapacidad y el rendimiento de los usuarios controlando el sistema BCI. Sin embargo, contrastan con el estudio de Piccione et al. (2006) que observó que el rendimiento se veía influido por el grado de discapacidad. Por otro lado, se estudió la correlación entre las puntuaciones de los tests de Luria (obtenidas para los usuarios del segundo grupo) y los resultados alcanzados por los usuarios controlando la aplicación. Únicamente se encontró relación entre la precisión conseguida en las sesiones de evaluación y la puntuación del test para la función de control atencional. Por lo tanto, los resultados sugieren que el grado de discapacidad no afectaría a la capacidad para controlar un sistema BCI basado en P300. No obstante, una capacidad de atención mínima podría ser el único requisito necesario para manejar adecuadamente estos sistemas, independientemente de la patología y el grado de discapacidad del usuario.

Existen estudios previos que analizaron sistemas BCI basados en P300 en poblaciones con discapacidad. En el caso de los estudios con deletreadores, Sellers and Donchin (2006) confirmaron que los sujetos con ELA en estadio avanzado podrían controlar sistemas BCI basados en P300. Más tarde, Nijboer et al. (2008) llevó a cabo 
un estudio exhaustivo de 40 semanas que demostró que la amplitud y latencia del potencial P300 permanecía estable durante ese periodo de tiempo. Más recientemente, los estudios de Ortner et al. (2011), Mak et al. (2012) y Spüler et al. (2012) mostraron que tanto los pacientes de ELA como las personas con discapacidad motora debida a otras patologías, podían llegar a controlar sistemas BCI basados en P300. Sin embargo, estos trabajos fueron llevados a cabo en poblaciones de pequeño tamaño comparado con el presente estudio y, además, se obtuvieron valores de precisión online y de ITR inferiores. En el caso de aplicaciones BCI basadas en P300 para control del entorno, existen dos estudios previos: Hoffman et al. (2008) y Aloise et al. (2011b). El primero propone un entorno simulado de control en el hogar, pero le analiza mediante una población de 4 sujetos que realizaron una única sesión experimental. En el segundo caso, se propone una aplicación de control del entorno virtual en la que participaron 7 sujetos y se llevaron a cabo dos sesiones experimentales. La novedad del estudio de Aloise et al. (2011b), es que propone un sistema BCI basado en P300 asíncrono. De esta forma, es capaz de detectar cuándo el usuario está ocupado en tareas mentales ajenas al sistema BCI, evitando así la ejecución de falsos positivos. Ambos trabajos propusieron entornos simulados o virtuales, a diferencia del presente estudio que se lleva a cabo con dispositivos reales y, además, en el entorno habitual de los usuarios. Además, las poblaciones bajo estudio de ambos trabajos son bastante más pequeñas y los valores de precisión online e ITR alcanzados son inferiores.

Si se compara la aplicación propuesta en el presente estudio con la aplicación BCI de control del entorno basada en MI (Corralejo et al. 2011), se observa que cuando se emplean potenciales P300 es posible presentar al usuario más dispositivos y opciones de control (8 dispositivos frente a 4 y 137 comandos de control frente a 19). Además, a diferencia de los sistemas basados en MI, las sesiones experimentales muestran que la mayoría de los usuarios alcanzan altos niveles de precisión con sistemas basados en P300. Por lo tanto, los resultados indican que los sistemas basados en P300 resultan mucho más adecuados para el desarrollo de aplicaciones BCI destinadas a personas con grave discapacidad. Finalmente, se observó también que el empleo de electrodos activos reduce mucho el tiempo de preparación evitando así la desmotivación que se producía en muchos usuarios durante el montaje de electrodos pasivos. 
Las puntuaciones otorgadas por los participantes en los cuestionarios indican que los usuarios valoraron favorablemente el diseño y la utilidad de la aplicación propuesta. Los participantes manifestaron que la herramienta era fácil de controlar y que las imágenes y el sistema de navegación a través de menús resultaba sencillo y fácil de entender. Además, consideraron que esta aplicación resulta realmente útil para incrementar su autonomía en el hogar. Incluso propusieron la inclusión de nuevos elementos como persianas, puertas y timbres para ser controlados mediante el sistema BCI. Sin embargo, muchos usuarios manifestaron que el registro de las señales EEG mediante un gorro y electrodos pasivos no resultaría práctico para el día a día. Como alternativa sugirieron el empleo de gorros inalámbricos y electrodos secos. Finalmente, la mayoría de los usuarios se sintieron satisfechos con su participación en el estudio. De hecho, se mostraron dispuestos a participar en futuros estudios.

Los resultados del presente estudio indican que las personas con grave discapacidad pueden controlar de forma adecuada los sistemas BCI basados en P300, incrementando así su autonomía personal. Sin embargo, el estudio presenta ciertas limitaciones. En primer lugar, aunque se emplea una población grande comparada con estudios previos de naturaleza similar, sería deseable incrementar el número de sujetos e incluir pacientes de ELA y personas con tetraplejia en futuros estudios. En segundo lugar, puesto que el rango de dispositivos existentes en nuestro entorno es muy amplio, se debería tratar de incorporar más dispositivos y funcionalidades explorando nuevas interfaces de control mediante Bluetooth, Wi-Fi o estándares de control domótico. De esta forma, sería posible satisfacer más necesidades del usuario final. Por otro lado, el desarrollo de mejoras algorítmicas favorecería un uso más frecuente de los sistemas BCI basados en P300: métodos fijos o dinámicos para detener las estimulaciones una vez identificado con precisión el elemento deseado; o paradigmas asíncronos que permitan al usuario decidir cuándo interactúa con la aplicación de forma autónoma. Así, se conseguirían sistemas mucho más prácticos para un uso diario en el entorno habitual. Finalmente, los usuarios finales no podrían poner en marcha el sistema BCI propuesto de forma autónoma. Aun así, este estudio da un paso importante al evaluar cómo una población grande de usuarios finales reales podría controlar un sistema autónomo basado en P300 en un momento dado. 


\section{C.7. Conclusiones}

\section{C.7.1. Contribuciones originales}

Las principales contribuciones de la Tesis recaen en el estudio y evaluación de una nueva aplicación BCI asistiva para control de dispositivos electrónicos en el hogar. Se trata del primer estudio en el que una amplia población de usuarios finales potenciales, personas con grave discapacidad, interactúan y evalúan una aplicación asistiva manejando dispositivos reales en su propio entorno. En el estudio participaron 30 sujetos con grave discapacidad. El estudio demuestra que estos sujetos pueden controlar sistemas BCI basados en potenciales P300 independientemente de su grado de discapacidad, aunque la capacidad de control atencional podría afectar a su rendimiento.

Las publicaciones a las que ha dado lugar la presente Tesis se indican en el Apéndice B de este documento. Las contribuciones originales de esta Tesis así como las ventajas de la metodología propuesta se indican a continuación:

1. Este es el primer estudio en el que participa un amplio grupo de sujetos con discapacidad. Estudios previos, especialmente los que se centraban en usuarios finales, emplearon poblaciones de menor tamaño. Sin embargo, un tamaño de población mayor permite obtener resultados más generalizables. Hasta donde conocemos, este es el primer estudio en el que participa una amplia población de personas con diferente grado de discapacidad, tanto motora como cognitiva, debido a diferentes patologías.

2. Se identificaron las principales necesidades de las personas con discapacidad. A continuación, la investigación se llevó a cabo considerando estas necesidades y deseos de los usuarios finales. Así, la aplicación BCI asistiva cubre las principales necesidades de confort, comunicación y entretenimiento.

3. En esta Tesis, se estudió una aplicación BCI asistiva basada en P300 para controlar dispositivos electrónicos en el hogar. Esta novedosa aplicación permite controlar 8 dispositivos: TV, reproductor de DVD, equipo de música, disco multimedia, luces, calefactor, ventilador y teléfono. Los dispositivos se controlan mediante comandos de control IR, empleando un dispositivo emisor de IR. La aplicación permite ejecutar 137 comandos (115 comandos de control IR y 22 comandos de navegación) distribuidos en 10 
matrices de imágenes (el menú principal y nueve sub-menús). Además, la aplicación propuesta emplea el patrón de estímulos por filas y columnas típico, pero atenuando los símbolos en lugar de iluminarlos, ya que experimentalmente se observó que el contraste se apreciaba mejor y resultaba menos molesto para los usuarios.

4. La metodología aplicada en esta Tesis incluye una evaluación inicial de todos los participantes. Los neuropsicólogos del NRC-D\&D valoraron el grado de discapacidad motora, de discapacidad cognitiva y la capacidad de atención sostenida de los 30 participantes. Además, el segundo grupo de usuarios (15 sujetos) realizó el test de Luria que valora nueve funciones cognitivas: percepción visual, orientación espacial, habla receptiva, habla expresiva, memoria inmediata, memorización lógica, imágenes temáticas, actividad conceptual y control atencional. Hasta donde conocemos, éste es el primer estudio en el que se ha llevado a cabo una valoración tan exhaustiva de los participantes.

5. Esta Tesis explora la posibilidad de que las personas con grave discapacidad puedan controlar un sistema BCI basado en P300 en su entorno habitual. La metodología consiste en la realización de tres sesiones experimentales: una primera sesión para calibrar y ajustar el clasificador para cada individuo; y dos sesiones adicionales para evaluar la capacidad de manejo de la aplicación por parte de los usuarios. Esta metodología permitió verificar que la mayoría de los participantes fue capaz de manejar la aplicación alcanzando precisiones superiores al 80\%.

6. La comparación entre los resultados alcanzados al manejar la aplicación y el grado de discapacidad y puntuaciones del test de Luria reafirman los resultados obtenidos en estudios previos con poblaciones de menor tamaño. Los resultados de esta Tesis confirman los resultados de Nijboer et al. (2008) ya que no se encontró correlación entre el rendimiento alcanzado y el grado de discapacidad. No obstante, sí que se encontró que la función de control atencional, medida por el test de Luria, tenía influencia sobre la precisión alcanzada durante las sesiones de evaluación. Esta capacidad de atención depende en algunos casos de la propia patología y de su estadio, por lo que coincide en parte con la conclusión obtenida por Piccione et al. 
(2006), que encontró relación entre el rendimiento y el grado de discapacidad.

7. En esta Tesis no solo el diseño sino también el procedimiento experimental y la evaluación se llevaron a cabo considerando a los usuarios finales. Los participantes completaron un cuestionario de evaluación tras terminar la etapa experimental para valorar diferentes aspectos de la aplicación BCI propuesta para incrementar la autonomía personal en el hogar. Esta Tesis da un paso importante al considerar las opiniones y sentimientos de los usuarios finales, así como su grado de satisfacción, ya que los estudios previos, en muchos casos, carecen del punto de vista de los usuarios.

\section{C.7.2. Conclusiones}

En la presente Tesis se investigó una nueva aplicación BCI asistiva basada en potenciales P300 para estudiar si podría resultar útil para control del entorno en personas con grave discapacidad. La aplicación propuesta permite controlar 8 dispositivos electrónicos reales, presentes habitualmente en el hogar, mediante 115 comandos de control IR y 22 comandos de navegación. La aplicación fue evaluada por una población formada por 30 usuarios finales potenciales de los sistemas BCI, personas con grave discapacidad a causa de diferentes patologías. Los usuarios interactuaron con el sistema BCI y los dispositivos de su entorno durante tres sesiones experimentales. 23 de los 30 usuarios fueron capaces de controlar la aplicación con una precisión superior al 80\% y 19 de ellos alcanzaron precisiones superiores al 95\%. Además, se obtuvieron tasas máximas de transferencia de información de hasta 25.9 bit/min. El análisis de los resultados sugiere que el grado de discapacidad no supone un problema a la hora de manejar apropiadamente la aplicación BCI basada en P300. Por lo tanto, estos sistemas podrían ser útiles para las personas con grave discapacidad, incrementando su autonomía personal en su entorno habitual. Además, resultaría bastante sencillo modificar la aplicación desarrollada para adaptarla a cada usuario e incrementar el número de necesidades que puede cubrir. Así, se podría reducir su dependencia respecto a cuidadores, familiares y enfermeros. 
Además de a esta conclusión global, los resultados de la investigación llevan a otras conclusiones específicas:

1. Las necesidades identificadas para la población bajo estudio se pueden agrupar en tres categorías principales: confort, comunicación y seguridad. Las necesidades de confort incluyen control del entorno, higiene y movilidad. Las necesidades relacionadas con accesibilidad y entretenimiento se engloban dentro de las necesidades de comunicación. Por último, las necesidades identificadas en el área de seguridad son las relacionadas con servicios de teleasistencia, detectores de caídas y sistemas de control de accesos.

2. Los sistemas BCI basados en potenciales P300 son los más adecuados para las personas con grave discapacidad ya que no necesitan período de entrenamiento y permiten seleccionar entre multitud de comandos de forma rápida. En concreto, la aplicación propuesta en este estudio permite ejecutar hasta 115 comandos de control IR de 8 dispositivos diferentes y 22 comandos de navegación. Los comandos se distribuyeron en diez matrices, un menú principal y nueve sub-menús, para simplificar la interfaz.

3. Los sistemas BCI basados en P300 resultan adecuados para aplicaciones asistivas ya que las personas con discapacidad podrían controlarlos adecuadamente. En el presente estudio, veintitrés de los treinta participantes alcanzaron precisiones superiores al 80\%. Estos resultados sugieren que la mayoría de los usuarios finales podrían llegar a manejar apropiadamente este tipo de sistemas sin consideraciones adicionales.

4. El grado de discapacidad, tanto motora como cognitiva, no tiene influencia sobre la capacidad de los sujetos a la hora de controlar la aplicación propuesta. No se encontró relación entre el rendimiento de los usuarios y su grado de discapacidad motora, de discapacidad cognitiva o de su capacidad de atención sostenida. Por lo tanto, la aplicación resulta apropiada para asistir a estas personas en el hogar incrementando su autonomía personal.

5. El análisis de los resultados mostró que existe una correlación entre la precisión alcanzada por los sujetos y la función de control atencional medida por el test de Luria. No obstante, los tests de Luria únicamente los 
realizaron los participantes del segundo grupo (15 sujetos), mientras que las valoraciones de la capacidad de atención sostenida estaban disponibles para la población total (30 sujetos) y para ésta no se encontró ninguna correlación. Por lo tanto, los resultados sugieren que el único requisito para controlar adecuadamente un sistema BCI basado en P300 sería la capacidad de atención de los sujetos, independientemente de su patología concreta y su grado de discapacidad.

6. La evaluación mediante cuestionarios llevada a cabo por los usuarios finales mostró que éstos están dispuestos a participar en este tipo de estudios, aportando sus necesidades y opiniones. Por ello, es conveniente centrarse en el usuario final a la hora de diseñar, desarrollar y evaluar futuros sistemas BCI.

7. La población bajo estudio se mostró satisfecha con la aplicación BCI diseñada y lo reflejó a través del cuestionario de evaluación. La mayoría de los participantes valoraron favorablemente el diseño y utilidad de la aplicación asistiva para su empleo en el entorno habitual.

8. A través del cuestionario de evaluación, la mayoría de los participantes coincidieron en la opinión de que la técnica de registro de las señales EEG, aun empleando electrodos activos, no resulta práctica para un uso diario de la aplicación. Los propios participantes sugirieron el empleo de gorros inalámbricos y electrodos secos para evitar este inconveniente. Estas opiniones han de ser consideradas en futuros desarrollos de aplicaciones BCI para usuarios finales.

\section{C.7.3. Líneas futuras}

En esta Tesis, se evaluó una novedosa aplicación BCI asistiva, que permite controlar dispositivos electrónicos, mediante una amplia población de usuarios finales reales. El objetivo era analizar si las personas con grave discapacidad podrían controlar adecuadamente este tipo de sistemas en su vida diaria. Los resultados muestran que la mayoría de los sujetos podrían controlar esta aplicación apropiadamente incrementando su autonomía en el hogar. A pesar de estos resultados prometedores, hay algunos aspectos que podrían ser abordados en futuros estudios. 
En primer lugar, debería considerarse la posibilidad de aumentar la población bajo estudio. La aplicación BCI propuesta y la metodología aplicada debería validarse en una base de datos de mayor tamaño para confirmar los resultados obtenidos en la Tesis. Aunque el tamaño de la población que ha participado en del presente estudio es mayor que la de estudios previos, debería tratar de incrementarse. Además, sería deseable que en futuros estudios participaran usuarios finales con otras patologías, como personas con ELA o tetraplejia.

La aplicación propuesta satisface diferentes necesidades relacionadas con el confort, la comunicación y el entretenimiento. Puesto que el rango de dispositivos existentes en nuestro entorno es muy amplio, se debería tratar de incorporar más dispositivos y funcionalidades mediante la inclusión de nuevos protocolos de control como Bluetooth, Wi-Fi o estándares de control domótico. De esta forma, se podrían cubrir más necesidades de los usuarios finales y adaptar la aplicación a cada usuario concreto.

Finalmente, para favorecer el uso frecuente de las aplicaciones BCI basadas en P300, sería interesante trabajar en nuevos algoritmos de procesado. Por un lado, se podrían investigar métodos para detener los estímulos una vez que se haya identificado con precisión el símbolo deseado por el usuario, permitiendo sistemas más eficientes (Schreuder et al. 2013). Por otro lado, resultaría verdaderamente interesante investigar paradigmas P300 asíncronos (Aloise et al. 2011a, 2011b) que permitan al usuario decidir de forma autónoma cuándo desea interactuar con la aplicación. Así, se podrían obtener sistemas BCI basados en P300 mucho más prácticos y eficientes para las personas con discapacidad en su entorno real. 



\section{Bibliography}

Alhaddad, M.J. (2012) Common Average Reference (CAR) Improves P300 Speller. International Journal of Engineering and Technology, vol. 2, no. 3, pp. 451-465.

Allison, B.Z., McFarland, D.J., Schalk, G., Zheng, S.D., Jackson, M.M. and Wolpaw, J.R. (2008) Towards an independent brain-computer interface using steady state visual evoked potentials. Clinical Neurophysiology, vol. 119, pp. 339408.

Allison, B.Z., Brunner, C., Kaiser, V., Müller-Putz, G.R., Neuper, C. and Pfurtscheller, G. (2010) Toward a hybrid brain-computer interface based on imagined movement and visual attention. Journal of Neural Engineering, vol. 7, 026007.

Aloise, F., Schettini, F., Aricò, P., Leotta, F., Salinari, S., Mattia, D., Babiloni, F. and Cincotti, F. (2011a) P300-based brain-computer interface for environmental control: an asynchronous approach. Journal of Neural Engineering, vol. 8, 025025.

Aloise, F., Schettini, F., Aricò, P., Salinari, S., Guger, C., Rinsma, J., Aiello, M., Mattia, D. and Cincotti, F. (2011b) Asynchronous P300-based brain-computer interface to control a virtual environment: initial tests on end users. Clinical EEG and Neuroscience, vol. 42, no. 4, pp. 219-224.

Bauernfeind, G., Leeb, R., Wriessnegger, S.C. and Pfurtscheller, G. (2008) Development, set-up and first results for a one-channel near-infrared spectroscopy system. Biomedizinische Technik / Biomedical Engineering, vol. 53, pp. 36-43. 
Bell, C.J., Shenoy, P., Chalodhom, R. and Rao, R.P.N. (2008) Control of a humanoid robot by a noninvasive brain-computer interface in humans. Journal of Neural Engineering, vol. 5, pp. 241-220.

Berger, H. (1929) Uber das Electrenkephalogramm des Menchen. Archiv für Psychiatrie und Nervenkrankheiten, vol. 87, pp. 527-570.

Birbaumer, N. (1997) Slow cortical potentials: their origin, meaning and clinical use. In: van Boxtel, G.J.M. and Böcker K.B.E. (eds.) Brain and behaviour past, present, and future (pp.25-39). Tilburg: Tilburg University Press.

Birbaumer, N., Kübler, A., Ghanayim, N., Hinterberger, T., Perelmouter, J., Kaiser, J., Iversen, I., Kotchoubey, B., Neumann, N. and Flor, H. (2000) The thought translation device (TTD) for completely paralized patients. IEEE Transactions on Rehabilitation Engineering, vol. 8, pp. 190-192.

Birbaumer, N. and Sauseng, P. (2010) Brain-Computer Interface in Neurorehabilitation. In: Graimann, B., Allison, B.Z. and Pfurtscheller, G. (eds.) Brain-Computer Interfaces. Revolutionizing Human-Computer Interaction (pp. 155-169). Berlin Heidelberg: Springer-Verlag.

Blankertz, B., Tangermann, M., Vidaurre, C., Dickhaus, T., Sannelli, C., Popescu, F., Fazli, S., Danóczy, M., Curio, G. and Müller, K.R. (2010) Detecting Mental States by Machine Learning Techniques: The Berlin Brain-Computer Interface. In: Graimann, B., Allison, B.Z. and Pfurtscheller, G. (eds.) BrainComputer Interfaces. Revolutionizing Human-Computer Interaction (pp. 113-135). Berlin Heidelberg: Springer-Verlag.

Bronzino, J.D. (2006) The Biomedical Engineering Handbook. 3rd ed. Boca Raton: Taylor \& Francis.

Brouwer, A.M. and van Erp, J.B.F. (2010) A tactile P300 brain-computer interface. Frontiers in Neuroscience, vol. 4, art. 19. 
Carrillo de la Peña, M.T. and Cadaveira, F. (2000) The effect of motivational instructions on P300 amplitude. Clinical Neurophysiology, vol. 30, no. 4, pp. 232-239.

Christensen, A.L. (1987) El diagnóstico neuropsicológico de Luria. 2nd ed. Madrid: Visor.

Citi, L., Poli, R., Cinel, C. and Sepulveda, F. (2008) P300-based BCI mouse with genetically-optimized analogue control. IEEE Transactions on Neural Systems and Rehabilitation Engineering, vol. 16, pp. 51-61.

Cohen, A. (2006) Biomedical signals: origin and dynamic characteristics; frequency domain analysis. In: Bronzino, J.D. (ed.) The Biomedical Engineering Handbook. Medical devices and systems. 3rd ed. Boca Raton: Taylor \& Francis.

Corralejo, R., Hornero, R. and Álvarez, D. (2011) A Domotic Control System Using Brain-Computer Interface (BCI), In: Proceedings of the $11^{\text {th }}$ International Work-Conference on Artificial Neural Networks (IWANN 2011), Part I, Lecture Notes in Computer Science, Torremolinos (Spain), vol. 6691, pp. 345-352.

Corralejo, R., Nicolás-Alonso, L.F., Álvarez, D. and Hornero, R. (2014) A P300-based brain-computer interface aimed at operating electronic devices at home for severely disabled people. Medical and Biological Engineering and Computing, vol. 52, no. 10, pp. 861-872.

Cui, X., Bray, S., Bryant, D.M., Glover, G.H. and Reiss A.L. (2011) A quantitative comparison of NIRS and fMRI across multiple cognitive tasks. Neuroimage, vol. 54, no. 4, pp. 2808-2821.

Dennett, D.C. (1992) Consciousness explained. Back Bay Books, Washington D.C.: Lippincott Williams \& Wilkins. 
Donchin, E. and Smith, D.B. (1970) The contingent negative variation and the late positive wave of the average evoked potential. Electroencephalography Clinical Neurophysiology, vol. 29, pp. 201-203.

Donchin, E., Spencer, K.M. and Wijesinghe, R. (2000) The mental prosthesis: assessing the speed of a P300-based brain-computer interface. IEEE Transactions on Rehabilitation Engineering, vol. 8, no. 2, pp. 174-179.

Donoghue, J.P. (2002) Connecting cortex to machines: recent advances in brain interfaces. Nature Neuroscience, vol. 5, 1085-1088.

Dornhege, G., Millan, J.D.R., Hinterberger, T., McFarland, D.J., Müller, K. and Sejnowski, T.J. (2007) Toward Brain-Computer Interfacing. Cambridge: The MIT Press.

Draper, N. and Smith, H. (1981) Applied Regression Analysis. New York: John Wiley and Sons.

Eliassen, J.C., Boespflug, E.L., Lamy, M., Allendorfer, J., Chu, W.J. and Szaflarski, J.P. (2008) Brain-Mapping Techniques for Evaluating Poststroke Recovery and Rehabilitation: A Review. Topics in Stroke Rehabilitation, vol. 15, no. 5, pp. 427-450.

Enderle, J.D., Bronzino, J.D. and Blanchard, S.M. (2005) Introduction to biomedical engineering. Burlington: Elsevier Academic Press.

Fabiani, G.E., McFarland, D.J., Wolpaw, J.R. and Pfurtscheller, G. (2004) Conversion of EEG activity into cursor movement by a brain-computer interface (BCI). IEEE Transactions on Neural Systems and Rehabilitation Engineering, vol. 12, pp. 331-338.

Farwell, L.A. and Donchin, E. (1988) Talking off the top of your head: toward a mental prosthesis utilizing event-related brain potentials. Electroencephalography and Clinical Neurophysiology, vol. 70, pp. 510-523. 
Fazel-Rezai, R. (2011) Recent Advances in Brain-Computer Interface System. InTech.

Fazel-Rezai, R., Allison, B.Z., Guguer, C., Sellers, E.W., Kleih, S.C. and Kübler, A. (2012) P300 brain computer interface: current challenges and emerging trends. Frontiers in Neuroscience, vol. 5, art. 14.

Fernández, J.M., Dauwalder, S., Torrellas, S., Faller, J., Scherer, R., Omedas, P., Verschure, P., Espinosa, A., Guger, C., Carmichael, C., Costa, U., Opisso, E., Tormos, J.M., Miralles, F. (2013) Connecting the disabled to their physical and social world: The BrainAble experience. In: TOBI Workshop IV Practical Brain-Computer Interfaces for End-Users: Progress and Challenges. Switzerland.

Finke, A., Lenhardt, A. and Ritter, H. (2009) The MindGame: A P300-based braincomputer interface game. Neural Networks, vol. 22, pp. 1329-1333.

Furdea, A., Halder, S., Krusienski, D.J., Bross, D., Nijboer, F., Birbaumer, N. and Kübler, A. (2009) An auditory oddball (P300) spelling system for braincomputer interfaces. Psychophysiology, vol. 46, pp. 617-625.

Graimann, B., Allison, B. and Pfurtscheller, G. (2010) Brain-Computer Interfaces: A Gentle Introduction. In: Graimann, B., Allison, B.Z. and Pfurtscheller, G. (eds.) Brain-Computer Interfaces. Revolutionizing Human-Computer Interaction (pp. 1-27). Berlin Heidelberg: Springer-Verlag.

Guger, C., Daban, S., Sellers, E., Holzner, C., Krausz, G., Carabalona, R., Gramatica, F. and Edlinger, G. (2009) How many people are able to control a P300-based brain-computer interface (BCI)? Neuroscience Letters, vol. 462, pp. 94-98.

Guger, C., Allison, B.Z. and Edlinger, G. (2013) Brain-Computer Interface Research: A State-of-the-Art Summary. Springer-Verlag.

Guger, C., Allison, B.Z. and Leuthardt, E.C. (2014a) Brain-Computer Interface Research: A State-of-the-Art Summary 2. Springer-Verlag. 
Guger, C., Vaughan, T. and Allison, B.Z. (2014b) Brain-Computer Interface Research: A State-of-the-Art Summary 3. Springer-Verlag.

Guger Technologies (2014) g.GAMMAsys, Guger Technologies [WWW] Available from: http://www.gtec.at/Products/Electrodes-and-Sensors/g.GAMMAsysSpecs-Features [Accessed 02/09/2014]. g.USBamp, Guger Technologies [WWW] Available from: http://www.gtec.at/Products/Hardware-andAccessories/g.USBamp-Specs-Features [Accessed 02/09/2014].

Guyon, I. and Elisseeff, A. (2003) An Introduction to Variable and Feature Selection. Journal of Machine Learning Research, vol. 3, pp. 1157-1182.

Hillyard, S.A. and Kutas, M. (1983) Electrophysiology of cognitive processing. Annual Review of Psychology, vol. 34, pp. 33-61.

Hoffman, U., Vesin, J.M., Ebrahimi, T. and Diserens, K. (2008) An efficient P300based brain-computer interface for disabled subjects. Journal of Neuroscience Methods, vol. 167, pp. 115-125.

Holz, E.M., Höhne, J., Staiger-Sälzer, P., Tangermann, M., Kübler, A. (2013) Braincomputer interface controlled gaming: evaluation of usability by severely motor restricted end-users. Artificial Intelligence in Medicine, vol 59, no. 2, pp. 111-120.

Hosmer, D.W. and Lemeshow, S. (1989) Applied Logistic Regression. New York: John Wiley and Sons.

Iturrate, I., Antelis, J.M., Kübler, A. and Mínguez, J. (2009) A Noninvasive brainactuated wheelchair based on a P300 neurophysiological protocol and automated navigation. IEEE Transactions on Robotics, vol. 25, pp. 614627.

Jasper, H.H. (1958) The ten twenty electrode system of the International Federation. Electroencephalography Clinical Neurophysiology, 10, pp. 371-375. 
Jin, J., Allison, B.Z., Sellers, E.W., Brunner, C., Horki, P., Wang, X. and Neuper, C. (2011) Optimized stimulus presentation patterns for an event-related potential EEG-based brain-computer interface. Medical and Biological Engineering and Computing, vol. 49 pp. 181-191.

Jin, J., Sellers, E.W. and Wang, X. (2012) Targeting an efficient target-to-target interval for P300 speller brain-computer interfaces. Medical and Biological Engineering and Computing, vol. 50, pp. 289-296.

Jurcak, V., Tsuzuki, D. and Dan, I. (2007) 10/20, 10/10, and 10/5 systems revisited: Their validity as relative head-surface-based positioning systems. NeuroImage, vol. 34, pp. 1600-1611.

Käthner, I., Daly, J., Halder, S., Räderscheidt, J., Armstrong, E., Dauwalder, S., Hintermüller, C., Espinosa, A., Vargiu, E., Pinegger, A., Faller, J., Wriessnegger, S.C., Miralles, F., Lowish, H., Markey, D., Müller-Putz, G.R., Martin, S., Kübler, A. (2014) A P300 BCI for e-inclusion, cognitive rehabilitation and smart home control. In: 6th International BCI Conference. Austria.

Khan, O.I., Farooq, F., Akram, F., Choi, M.T., Han, S., Kim, T.S. (2012) Robust extraction of P300 using constrained ICA for BCI applications. Medical and Biological Engineering and Computing, vol. 50, pp. 231-241.

Kleih, S.C., Nijboer, F., Halder, S. and Kübler, A. (2010) Motivation modulates the P300 amplitude during brain-computer interface use. Clinical Neurophysiology, vol. 121, pp. 1023-1031.

Klobassa, D.S., Vaughan, T.M., Brunner, P., Schwartz, N.E., Wolpaw, J.R., Neuper, C. and Sellers, E.W. (2009) Toward a high-throughput auditory P300-based brain-computer interface. Clinical Neurophysiology, vol. 120, pp. 12521261. 
Krusienski, D.J., Sellers, E.W., Cabestaing, F., Bayoudh, S., McFarland, D.J., Vaughan, T.M. and Wolpaw, J.R. (2006) A comparison of classification techniques for the P300 speller. Journal of Neural Engineering, vol. 3, pp. 299-305.

Krusienski, D.J., Sellers, E.W., McFarland, D.J., Vaughan, T.M. and Wolpaw, J.R. (2008) Toward enhanced P300 speller performance. Journal of Neuroscience Methods, vol. 167, pp. 14-21.

Kübler, A., Kotchoubey, B., Hintergerger, T., Ghanayim, N., Perelmouter, J., Schauer, M., Fritsch, C., Taub, E. and Birbaumer, N. (1999) The thought translation devices: a neurophysiological approach to communication in total motor paralysis. Experimental Brain Research, vol. 124, pp. 223 - 232.

Kübler, A., Kotchoubey, B., Kaiser, J., Wolpaw, J.R. and Birbaumer, N. (2001) Braincoputer communiation: unlock the locked-in. Psychological Bulletin, vol. 127, no. 3, pp. 358-375.

Laguna, P. and Sörnmo, L. (2009) Introduction. Editorial on Signal processing in vital rhythms and signs. Philosophical Transactions of the Royal Society A: Mathematical, Physical and Engineering Sciences, vol. 367, pp. 207-211.

Levine, S.P., Huggins, J.E., BeMent, S.L., Kushwaha, R.K., Schuh, L.A., Passaro, E.A., Rohde, M.M. and Ross, D.A. (1999) Identification of electrocorticogram patterns as the basis for a direct brain interface. Journal of Clinical Neurophysiology, vol. 16, pp. 439-447.

Maestú, C., Gómez-Utrero, E., Piñeiro, R. and Sola, R.G. (1999) Magnetoencefalografía: una nueva técnica de diagnóstico funcional en neurociencia. Revista de Neurología, vol. 28, no. 11, pp. 1077-1090.

Mak, J.N., Arbel, Y., Minett, J.W., McCane, L.M., Yuksel, B., Ryan, D., Thompson, D., Bianchi, L. and Erdogmus, D. (2011) Optimizing the P300-based braincomputer interface: current status, limitations and future directions. Journal of Neural Engineering, vol. 8, 025003. 
Mak, J.N., McFarland, D.J., Vaughan, T.M., McCane, L.M., Tsui, P.Z., Zeitlin, D.J., Sellers, E.W. and Wolpaw, J.R. (2012) EEG correlates of P300-based brain-computer interface (BCI) performance in people with amyotrophic lateral sclerosis. Journal of Neural Engineering, vol. 9, 026014.

Mao, K.Z. (2002) Fast Orthogonal Forward Selection Algorithm for Feature Subset Selection. IEEE Transactions on Neural Networks, vol. 13, no. 5, pp. 12181224.

Martí, J. (1988) Medidas en el sistema nervioso y muscular. In: Mompín, J. (ed.) Introducción a la Bioingeniería. Barcelona: Marcombo Boixareu.

McFarland, D.J., McCane, L.M., David, S.V. and Wolpaw, J.R. (1997) Spatial filter Selection for EEG-based communication. Electroencephalography and Clinical Neurophysiology, vol. 103, pp. 386-394.

McFarland, D.J., Krusienski, D.J., Sarnacki, W.A. and Wolpaw, J.R. (2008) Emulation of computer mouse control with a noninvasive brain-computer interface. Journal of Neural Engineering, vol. 5, no. 2 , pp. 101-110.

Miller, K.J. and Ojemann, J.G. (2010) A Simple, Spectral-Change Based, Electrocorticographic Brain-Computer Interface. In: Graimann, B., Allison, B.Z. and Pfurtscheller, G. (eds.) Brain-Computer Interfaces. Revolutionizing Human-Computer Interaction (pp. 241-258). Berlin Heidelberg: SpringerVerlag.

Münßinger, J.I., Halder, S., Kleih, S.C., Furdea, A., Raco, V., Hösle, A. and Kübler, A. (2010) Brain painting: first evaluation fo a new brain-computer interface application with ALS-patients and healthy volunteers. Frontiers in Neuroscience, vol. 4, 182.

Mugler, E.M., Ruf, C.A., Halder, S., Bensch, M. and Kübler, A. (2010) Design and implementation of a P300-based brain-computer interface for controlling an internet browser. IEEE Transactions on Neural Systems and Rehabilitation Engineering, vol. 18, pp. 599-609. 
Müller-Putz, G.R., Scherer, R., Neuper, C. and Pfurtscheller, G. (2006) Steady-state somatosensory evoked potentials: suitable brain signals for brain-computer interfaces? IEEE Transactions on Neural Systems and Rehabilitation Engineering, vol. 14, pp. 30-37.

Nebeker, F. (2002) Golden accomplishments in biomedical engineering. IEEE Engineering in Medicine and Biology Magazine, vol. 21, pp. 17-47.

Neuper, C, and Pfurtscheller, G. (2010) Neurofeedback Training for BCI Control. In: Graimann, B., Allison, B.Z. and Pfurtscheller, G. (eds.) Brain-Computer Interfaces. Revolutionizing Human-Computer Interaction (pp. 65-78). Berlin Heidelberg: Springer-Verlag.

Niedermeyer, E. and Silva, F.L.D. (2005) Electroencephalography: Basic principles, clinical applications, and related fields. Philadelphia: Lippincott Williams \& Wilkins.

Nijboer, F., Sellers, E.W., Mellinger, J., Jordan, M.A., Matuz, T., Furdea, A. and Halder, S. (2008) A P300-based brain-computer interface for people with amyotrophic lateral sclerosis. Clinical Neurophysiology, vol. 119, pp. 19091916.

Nijboer, F. and Broermann, U. P. (2010) Brain-Computer Interfaces for Communication and Control in Locked-in Patients. In: Graimann, B., Allison, B.Z. and Pfurtscheller, G. (eds.) Brain-Computer Interfaces. Revolutionizing HumanComputer Interaction (pp. 185-201). Berlin Heidelberg: Springer-Verlag.

Ortner, R., Aloise, F., Prückl, R., Schettini, F., Putz, V., Scharinger, J., Opisso, E., Costa, U. and Guger, C. (2011) Accuracy of a P300 speller for people with motor impairments: a comparison. Clinical EEG and Neuroscience, vol. 42, no. 4, pp. 214-218.

Perelmouter, J. and Birbaumer, N. (2000) A binary spelling interface with random errors. IEEE Transactions on Rehabilitation Engineering, vol. 8, no. 2, pp. 227-232. 
Pfurtscheller, G., Brunner, C., Leeb, R., Scherer, R., Müller-Putz, G.R. and Neuper, C. (2010) The Graz Brain-Computer Interface. In: Graimann, B., Allison, B.Z. and Pfurtscheller, G. (eds.) Brain-Computer Interfaces. Revolutionizing Human-Computer Interaction (pp. 79-96). Berlin Heidelberg: SpringerVerlag.

Pineda, J.A., Allison, B.Z. and Vankov, A. (2000) The effects of Self-Movement, Observation, and Imagination on Rhythms and Readiness Potentials (RP’s): Toward a Brain-Computer Interface (BCI). IEEE Transactions on Rehabilitation Engineering, vol. 8, no. 2, pp. 219-222.

Piccione, F., Giorgi, F., Tonin, P., Priftis, K., Giove, S., Silvoni, S., Palmas, G. and Beverina, F. (2006) P300-based brain computer interface: reliability and performance in healthy and paralysed participants. Clinical Neurophysiology, vol. 117, pp. 531-537.

Poza, J. (2008) Análisis Tiempo-Frecuencia de la Actividad Magnetoencefalográfica espontánea en la enfermedad de Alzheimer. PhD Thesis, University of Valladolid, Valladolid, Spain.

Pritchard, W.S. (1981) Psychophysiology of P300. Psychologycal Bulletin, vol. 89, no.3, pp. 506-540.

Rakotomamonjy, A. and Guigue, V. (2008) BCI competition III: dataset II - ensemble of SVMs for BCI P300 speller. IEEE Transactions on Biomedical Engineering, vol. 55, pp. 1147-1154.

RedRat (2013) RedRat Website. [WWW] Available from: http://www.redrat.co.uk [Accessed: 11/06/2013].

Rivet, B., Souloumiac, A., Attina, V. and Gibert, G. (2009) xDAWN algorithm to enhance evoked potentials: application to brain-computer interface. IEEE Transactions on Biomedical Engineering, vol. 56, pp. 2035-2043. 
Rockstroh, B., Elbert, T., Canavan, A., Lutzenberger, W. and Birbaumer, N. (1989) Slow cortical potentials and behaviour. 2nd ed. Baltimore: Urban and Schwarzenberg.

Salvaris, M. and Sepulveda, F. (2009) Visual modifications on the P300 speller BCI paradigm. Journal of Neural Engineering, vol. 6, 046011.

Schalk, G., McFarland, D.J., Hinterberger, T., Birbaumer, N. and Wolpaw, J.R. (2004) BCI2000: a general-prupose brain-computer interface (BCI) system. IEEE Transactions on Biomedical Engineering, vol. 51, no. 6, pp. 1034-1043.

Schalk, G. (2006) Towards a clinically practical brain-computer interface. PhD Thesis, Faculty of Rensselaer Polytechnic Institute, Troy, New York, United States of America.

Schalk, G. (2007) Topic: Electrode placement. BCI2000 Community Forum [WWW] Available from: http://www.bci2000.org/phpbb/viewtopic.php?f=3\&t=390 [Accessed: 15/12/2013].

Schalk, G. and Mellinger, J. (2010) A Practical Guide to Brain-Computer Interfacing with BCI2000. London: Springer-Verlag.

Schlögl, A., Lugger, K. and Pfurtscheller, G. (1997) Using adaptive autoregressive parameters for a brain-computer-interface experiment. In: Proceedings of the $19^{\text {th }}$ International Conference of the IEEE EMBS, Chicago, October 1997. Chicago: IEEE, pp. 1533-1535.

Schlögl, A., Lee, F., Bischof, H. and Pfurtscheller, G. (2005) Characterization of fourclass motor imagery EEG data for the BCI-competition 2005. Journal of Neural Engineering, vol. 2, L14-L22.

Schreuder, M., Höhne, J., Blankertz, B., Haufe, S., Dickhaus, T. and Tangermann, M. (2013) Optimizing event-related potential based brain-computer interfaces: a systematic evaluation of dynamic stopping methods. Journal of Neural Engineering, vol. 10, 036025. 
Schwartz, A.B. (2004) Cortical neural prosthetics. Annual Review of Neuroscience, vol. 27, pp. 487-507.

Sellers, E.W. and Donchin, E. (2006) A P300-based brain-computer interface: initial tests by ALS patients. Clinical Neurophysiology, vol. 117, pp. 538-548.

Sellers, E.W., Krusienski, D.J., McFarland, D.J., Vaughan, T.M. and Wolpaw, J.R. (2006) A P300 event-related potential brain-computer interface (BCI): the effects of matrix size and inter stimulus interval on performance. Biological Psychology, vol. 73, pp. 242-252.

Sellers, E.W., McFarland, D.J., Vaughan, T.M. and Wolpaw, J.R. (2010) BCIs in the Laboratory and at Home: The Wadsworth Research Program. In: Graimann, B., Allison, B.Z. and Pfurtscheller, G. (eds.) Brain-Computer Interfaces. Revolutionizing Human-Computer Interaction (pp. 97-111). Berlin Heidelberg: Springer-Verlag.

Serby, H., Yom-Tov, E. and Inbar, G.F. (2005) An improved P300-based braincomputer interface. IEEE Transactions on Neural Systems and Rehabilitation Engineering, vol. 13, pp. 89-98.

Spüler, M., Bensch, M., Kleih, S., Rosenstiel, W., Bogdan, M. and Kübler, A. (2012) Online use of error-related potentials in healthy users and people with severe motor impairment increases performance of a P300-BCI. Clinical Neurophysiology, vol. 123, pp. 1328-1337.

Sutter, J.M. and Kalivas, J.H. (1993) Comparison of Forward Selection, Backward Elimination, and Generalized Simulated Annealing for Variable Selection. Microchemical Journal, vol. 47, pp. 60-66.

Sutton, S., Braren, M., Zubin, J. and John, E.R. (1965) Evoked correlates of stimulus uncertainty. Science, vol. 150, pp. 1187-1188.

Taylor, D.M. and Stetner, M.E. (2010) Intracortical BCIs: A Brief History of Neural Timing. In: Graimann, B., Allison, B.Z. and Pfurtscheller, G. (eds.) Brain- 
Computer Interfaces. Revolutionizing Human-Computer Interaction (pp. 203-219). Berlin Heidelberg: Springer-Verlag.

Townsend, G., LaPallo, B.K., Boulay, C.B., Krusienski, D.J., Frye, G.E., Hauser, C.K., Schwartz, N.E., Vaughan, T.M., Wolpaw, J.R. and Sellers, E.W. (2010) A novel P300-based brain-computer interface stimulus presentation paradigm: Moving beyond rows and columns. Clinical Neurophysiology, vol. 121, pp. 1109-1120.

Vidal, J.J. (1977) Real-Time Detection of Brain Events in EEG. Proceedings of the IEEE, vol. 65, no. 5, pp. 633-641.

Walter, W.G., Cooper, R., Aldridge, V.J., McCallum, W.C. and Winter, A.L. (1964) Contingent negative variation: an electric sign of sensorimotor association and expectancy in the human brain. Nature, vol. 203, pp. 380-384.

Wolpaw, J.R., Birbaumer, N., Heetderks, W.J., McFarland, D.J., Peckham, P.H., Schalk, G., Donchin, E., Quatrano, L.A., Robinson, C.J. and Vaughan, T.M. (2000) Brain-Computer Interface Technology: A Review of the First International Meeting. IEEE Transactions on Rehabilitation Engineering, vol. 8, no. 2, pp. 164-173.

Wolpaw, J.R., Birbaumer, N., McFarland, D.J., Pfurtscheller, G. and Vaughan, T.M. (2002) Brain-computer interfaces for communication and control. Clinical Neurophysiology, vol. 113, pp. 767-791.

Wolpaw, J.R., Loeb, G.E., Allison, B.Z., Donchin, E., do Nascimento, O.F., Heetderks, W.J., Nijboer, F., Shain, W.G. and Turner, J.N. (2006) BCI Meeting 2005 Workshop on signals and recording methods. IEEE Transactions on Neural Systems and Rehabilitation Engineering, vol. 14, pp. 138-141.

Xu, N., Gao, X., Hong, B., Miao, X., Gao, S. and Yang, F. (2004) BCI competition 2003—data set IIb: enhancing P300 wave detection using ICA-based subspace projections for BCI applications. IEEE Transactions on Biomedical Engineering, vol. 51, pp. 1067-1072. 
Yu, T., Li, Y., Long, J. and Gu, Z. (2012) Surfing the internet with a BCI mouse. Journal of Neural Engineering, vol. 9, 036012. 